\title{
Enantioselective Synthesis of N1999A2
}

Nan Ji, Hardwin O’Dowd, Brad M. Rosen, and Andrew G. Myers*

J. Am. Chem. Soc.

\section{Supporting Information}

Index

General Experimental Procedures

Materials

Instrumentation

Synthetic Procedures

General Experimental Procedures. All reactions were performed in oven- or flame-dried round-bottom or modified Schlenk flasks fitted with rubber septa under a positive pressure of argon, unless otherwise noted. Air- and moisture-sensitive liquids and solutions were transferred via syringe or stainless steel cannula. Where necessary (so noted), solutions were deoxygenated by alternate freeze (liquid nitrogen)/evacuation/thaw cycles ( $\geq$ three iterations). Organic solutions were concentrated by rotary evaporation (house vacuum, $\sim 25$ Torr) at $23-30{ }^{\circ} \mathrm{C}$. Flash-column chromatography was performed as described by Still et al., ${ }^{1}$ employing silica gel (60- $\AA$ pore size,

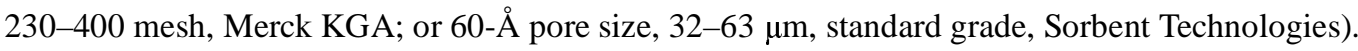
Analytical thin-layer chromatography (TLC) was performed using glass plates pre-coated with silica gel (0.25 mm, 60- $\AA$ pore size, 230-400 mesh, Merck KGA) impregnated with a fluorescent indicator $(254 \mathrm{~nm})$. TLC plates were visualized by exposure to ultraviolet light (UV) and/or exposure to ceric ammonium molybdate solution (CAM) or an acidic solution of $p$-anisaldehyde (anisaldehyde) followed by brief heating on a hot plate $\left(200{ }^{\circ} \mathrm{C}, 10-15 \mathrm{~s}\right)$.

Materials. Commercial reagents and solvents were used as received unless mentioned otherwise. Dichloromethane, ether, tetrahydrofuran, $N, N$-dimethylformamide and toluene were purified by the method of Pangborn et al. ${ }^{2}$ The molarity of solutions of $n$-butyllithium and $t$-butyllithium was determined by titration against a standard solution of 2-butanol in tetrahydrofuran using triphenylmethane as an indicator (average of three determinations). ${ }^{3}$

Instrumentation. Proton nuclear magnetic resonance $\left({ }^{1} \mathrm{H}\right.$ NMR) spectra and carbon nuclear magnetic resonance $\left({ }^{13} \mathrm{C} \mathrm{NMR}\right)$ were recorded with Varian Unity/Inova $600(600 \mathrm{MHz})$, Varian Unity/ Inova 500 (500 MHz/125 MHz), or Varian Mercury 400 (400 MHz/100 MHz) NMR spectrometers. Chemical shifts for protons are reported in parts per million scale $(\delta$ scale) downfield from tetramethylsilane and are referenced to residual protium in the NMR solvents $\left(\mathrm{CHCl}_{3}: \delta 7.26, \mathrm{C}_{6} \mathrm{D}_{5} \mathrm{H}: \delta 7.15, \mathrm{CD}_{2} \mathrm{HCN}: 1.93\right)$. Chemical shifts for carbon are reported in parts per million ( $\delta$ scale) downfield from tetramethylsilane and are referenced to the carbon resonances of the solvent $\left(\mathrm{CDCl}_{3}: \delta 77.0, \mathrm{C}_{6} \mathrm{D}_{5} \mathrm{H}: \delta\right.$ 128.0). Data are represented as follows: chemical shift, multiplicity ( $\mathrm{s}=$ singlet, $\mathrm{d}=$ doublet, $\mathrm{t}=$ triplet, $\mathrm{q}=$ quartet, $\mathrm{m}=$ multiplet, $\mathrm{br}=$ broad $)$, integration,

(1) Still, W. C.; Kahn, M.; Mitra, A. J. Org. Chem. 1978, 43, 2923.

(2) Pangborn, A. B.; Giardello, M. A.; Grubbs, R. H.; Rosen, R. K.; Timmers, F. J. Organometallics 1996, $15,1518$.

(3) Duhamel, L.; Palquevent, J.-C. J. Org. Chem. 1979, 44, 3404. 
coupling constant in Hz, and assignment. Infrared (IR) spectra were obtained using a Perkin-Elmer 1600 FT-IR spectrophotometer referenced to a polystyrene standard. High resolution mass spectra were obtained at the Harvard University Mass Spectrometry Facilities.

\section{Synthetic Procedures.}

(For clarity, intermediates that have not been assigned numbers in the text are numbered sequentially in the Supporting Information beginning with 27.)

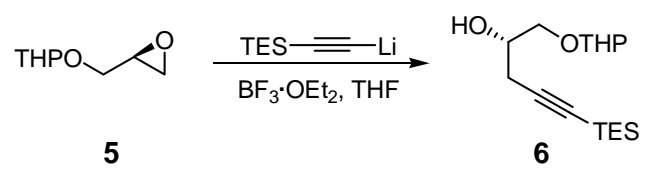

\section{$\underline{\text { Alcohol } 6}$}

A solution of lithium bis(trimethysilyl)amide in tetrahydrofuran $(1.0 \mathrm{M}, 100 \mathrm{~mL}, 0.100$ mol, 1.2 equiv) was added dropwise via cannula over $20 \mathrm{~min}$ to a solution of (triethylsilyl)acetylene $\left(14.0 \mathrm{~g}, 17.9 \mathrm{~mL}, 0.100 \mathrm{~mol}, 1.2\right.$ equiv) in tetrahydrofuran $(100 \mathrm{~mL})$ at $\quad-78^{\circ} \mathrm{C}$. The resulting solution was stirred at $-78{ }^{\circ} \mathrm{C}$ for $10 \mathrm{~min}$. Boron trifluoride etherate $(12.7 \mathrm{~mL}, 0.100 \mathrm{~mol}, 1.2$ equiv) was then added via syringe over $3 \mathrm{~min}$. After $20 \mathrm{~min}$, a cold $\left(-78{ }^{\circ} \mathrm{C}\right)$ solution of the THP-protected $(R)-(+)$-glycidol (5, an inseparable, 1:1 mixture of diastereomers, THP acetal epimers $)^{4}(13.2 \mathrm{~g}, 0.083 \mathrm{~mol}, 1.0$ equiv) in tetrahydrofuran $(70 \mathrm{~mL})$ was added dropwise to the cold $\left(-78^{\circ} \mathrm{C}\right)$ reaction solution via cannula over $10 \mathrm{~min}$. After $3 \mathrm{~h}$, saturated aqueous ammonium chloride solution $(150 \mathrm{~mL})$ was added to quench any excess base. Tetrahydrofuran was removed in vacuo. The residue was extracted with ether $(2 \times 200 \mathrm{~mL})$. The organic extracts were combined and the combined solution was washed with water $(100 \mathrm{~mL})$. The organic layer was separated and washed with brine $(100 \mathrm{~mL})$. The washed organic solution was dried over anhydrous sodium sulfate. The dried solution was filtered and the filtrate was concentrated to provide the crude alcohol $\mathbf{6}$ as an inseparable, 1:1 mixture of diastereomers (THP acetal epimers, colorless oil, $24.8 \mathrm{~g}, 100 \%$ ). The crude product was used in the next reaction without purification.

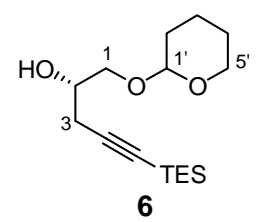

$\mathrm{R}_{f}=0.20$ (20\% ether-hexanes). ${ }^{1} \mathrm{H}$ NMR (1:1 mixture of diastereomers, THP acetal epimers, 400 MHz, $\mathrm{CDCl}_{3}$ ), $\delta$ 4.59-4.54 (m, J = 4.4 Hz, 1H, C(1')-H), 3.96-3.87 (m, 3H, C(2)-H + C(5')-H), $3.83\left(\mathrm{dd}, \mathrm{J}_{1}=3.6 \mathrm{~Hz}, \mathrm{~J}_{2}=10.8 \mathrm{~Hz}, 0.5 \mathrm{H}, \mathrm{C}(1)-\mathrm{H}\right), 3.77\left(\mathrm{dd}, \mathrm{J}_{1}=3.2 \mathrm{~Hz}, \mathrm{~J}_{2}=10.4 \mathrm{~Hz}, 0.5 \mathrm{H}\right.$, $\mathrm{C}(1)-\mathrm{H}), 3.67$ (dd, $\left.\mathrm{J}_{1}=6.8 \mathrm{~Hz}, \mathrm{~J}_{2}=10.8 \mathrm{~Hz}, 0.5 \mathrm{H}, \mathrm{C}(1)-\mathbf{H}\right), 3.59$ (dd, $\mathrm{J}_{1}=6.0 \mathrm{~Hz}, \mathrm{~J}_{2}=10.4 \mathrm{~Hz}, 0.5$ H, C(1)-H), 3.55-3.49 (m, 2H, C(5')-H), 3.19 (d, J = 4.0 Hz, 0.5 H, C(2)-OH), 2.88 (d, J = 5.6 Hz, 0.5 H, C(2)-OH), 2.57-2.41 (m, 2H, C(3)-CH), 1.86-1.50 (m, 6H, C(2', '3', '4')-H), 0.97 (t, J = 7.6 $\left.\mathrm{Hz}, 9 \mathrm{H}, \mathrm{Si}\left(\mathrm{CH}_{2} \mathrm{CH}_{3}\right)_{3}\right), 0.56\left(\mathrm{q}, \mathrm{J}=7.6 \mathrm{~Hz}, 6 \mathrm{H}, \mathrm{Si}\left(\mathrm{CH}_{2} \mathrm{CH}_{3}\right)_{3}\right) .{ }^{13} \mathrm{C} \mathrm{NMR}\left(100 \mathrm{MHz}, \mathrm{CDCl}_{3}\right), \delta$ 103.8, 103.7, 100.0, 99.9, 84.3 (2), 72.0, 71.1, 69.2, 69.1, 63.0, 62.9, 30.6 (2), 25.2 (2), 25.1, 24.8, 19.8 (2), 7.4, 4.4. IR ( $\mathrm{NaCl}$, thin film) $\mathrm{cm}^{-1} 3438,2953,2895,2875,2733,2174,1456,1430$, 1415, 1384, 1353, 1324, 1262, 1237, 1202, 1183, 1125, 1078, 1035, 972, 908, 870, 816, 728.

\footnotetext{
${ }^{4}$ For preparation of 5, see: Meng, D.; Bertinato, P.; Balog, A.; Su, D.-S.; Kamenecka, T.; Sorensen, E. J.; Danishefsky, S. J. J. Am. Chem. Soc, 1997, 119, 10073.
} 
HRMS-ESI $(\mathrm{m} / z):[\mathrm{M}+\mathrm{H}]^{+}$calcd for $\mathrm{C}_{16} \mathrm{H}_{31} \mathrm{O}_{3} \mathrm{Si}, 299.2042$; found 299.2046 .

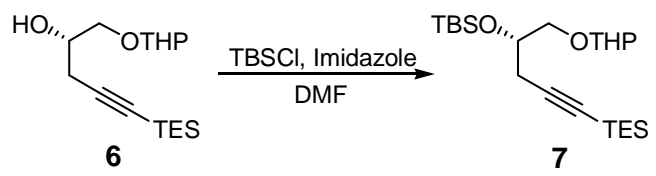

\section{tert-Butyldimethylsilyl Ether 7}

tert-Butyldimethylsilyl chloride ( $25.2 \mathrm{~g}, 0.125 \mathrm{~mol}, 2.0$ equiv) was added to a stirring solution of the alcohol 6 ( $24.8 \mathrm{~g}, 0.083 \mathrm{~mol}, 1.0$ equiv) and imidazole ( $15.0 \mathrm{~g}, 0.250 \mathrm{~mol}, 3.0$ equiv) in $N, N$-dimethylformamide $(200 \mathrm{~mL})$ at $23{ }^{\circ} \mathrm{C}$. After stirring at $23{ }^{\circ} \mathrm{C}$ for $17 \mathrm{~h}$, the reaction mixture was partitioned between ether $(1 \mathrm{~L})$ and water $(500 \mathrm{~mL})$. The organic layer was separated and washed successively with water $(500 \mathrm{~mL}), 1 \mathrm{~N}$ aqueous hydrochloric acid solution $(300 \mathrm{~mL})$, saturated aqueous sodium bicarbonate solution $(300 \mathrm{ml})$, and brine $(200 \mathrm{~mL})$. The washed solution was then dried over anhydrous sodium sulfate. The dried solution was filtered and the filtrate was concentrated. The residue was purified by flash-column chromatography (5\% ethyl acetate-hexanes) to give the tert-butyldimethylsilyl ether 7 (yellow oil, $32.6 \mathrm{~g}, 95 \%$, two steps).

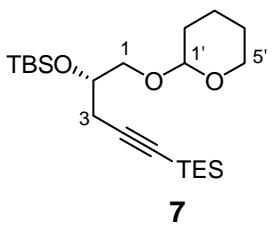

$\mathrm{R}_{f}=0.25$ (5\% ethyl acetate-hexanes). ${ }^{1} \mathrm{H}$ NMR (1:1 mixture of diastereomers, $600 \mathrm{MHz}, \mathrm{CDCl}_{3}$ ),

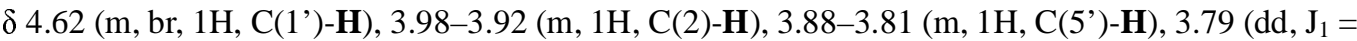
$\left.4.8 \mathrm{~Hz}, \mathrm{~J}_{2}=10.2 \mathrm{~Hz}, 0.5 \mathrm{H}, \mathrm{C}(1)-\mathbf{H}\right), 3.70$ (dd, $\left.\mathrm{J}_{1}=4.8 \mathrm{~Hz}, \mathrm{~J}_{2}=10.2 \mathrm{~Hz}, 0.5 \mathrm{H}, \mathrm{C}(1)-\mathbf{H}\right), 3.50-3.47$ $\left(\mathrm{m}, 1 \mathrm{H}, \mathrm{C}\left(5^{\prime}\right)-\mathbf{H}\right), 3.42\left(\mathrm{dd}, \mathrm{J}_{1}=5.4 \mathrm{~Hz}, \mathrm{~J}_{2}=10.2 \mathrm{~Hz}, 0.5 \mathrm{H}, \mathrm{C}(1)-\mathbf{H}\right), 3.31\left(\mathrm{dd}, \mathrm{J}_{1}=6.6 \mathrm{~Hz}, \mathrm{~J}_{2}=9.6\right.$ $\mathrm{Hz}, 0.5 \mathrm{H}, \mathrm{C}(1)-\mathbf{H}$ ), 2.54-2.47 (2 diastereomers, dd (2), $\left.\mathrm{J}_{1}=6.0 \mathrm{~Hz}, \mathrm{~J}_{2}=18.6 \mathrm{~Hz}, 1 \mathrm{H}, \mathrm{C}(3)-\mathbf{H}\right)$, 2.42-2.37 (2 diastereomers, dd (2), $\left.\mathrm{J}_{1}=6.0 \mathrm{~Hz}, \mathrm{~J}_{2}=18.6 \mathrm{~Hz}, 2 \mathrm{H}, \mathrm{C}(3)-\mathbf{H}\right), 1.85-1.79(\mathrm{~m}, 1 \mathrm{H}$, C(2')-H), 1.72-1.67 (m, 1H, C(2')-H), 1.62-1.49 (m, 4H, C(3')-H + C(4')-H), 0.97 (t, J = 7.8 Hz, $\left.9 \mathrm{H}, \mathrm{Si}\left(\mathrm{CH}_{2} \mathrm{CH}_{3}\right)_{3}\right), 0.89$ (2 diastereomers, s, 9H, $\left.\mathrm{Si}\left(\mathrm{CH}_{3}\right)_{2} \mathrm{C}\left(\mathrm{CH}_{3}\right)_{3}\right), 0.56$ (q, J = 7.8 Hz, 6H, $\left.\mathrm{Si}\left(\mathrm{CH}_{2} \mathrm{CH}_{3}\right)_{3}\right), 0.10$ (2 diastereomers, s, 3H, $\left.\mathrm{Si}\left(\mathrm{CH}_{3}\right)_{2} \mathrm{C}\left(\mathrm{CH}_{3}\right)_{3}\right), 0.09$ (s, 3H, $\mathrm{Si}\left(\mathrm{CH}_{3}\right)_{2} \mathrm{C}\left(\mathrm{CH}_{3}\right)_{3}$ ), 0.07 (s, 3H, $\left.\mathrm{Si}\left(\mathrm{CH}_{3}\right)_{2} \mathrm{C}\left(\mathrm{CH}_{3}\right)_{3}\right) .{ }^{13} \mathrm{C}$ NMR $\left(100 \mathrm{MHz}, \mathrm{CDCl}_{3}\right), \delta$ 105.0, 104.9, 99.0, 98.6, 83.4, 83.3, 70.8, 70.6, 70.5, 70.4, 61.8, 61.6, 30.5, 30.4, 26.2 , 26.1, 25.8, 25.5, 25.4, 19.2, 19.1, 18.1 (2), 7.4, 4.4, -4.6 (2), -4.7. IR (NaCl, thin film) $\mathrm{cm}^{-1} 3448,2954,2875,2857,2176,1654,1637$, 1463, 1447, 1415, 1361, 1255, 1202, 1125, 1067, 1037, 939, 908, 837, 778, 720. HRMS-CI $(\mathrm{m} / \mathrm{z})$ $\left[\mathrm{M}+\mathrm{NH}_{4}\right]^{+}$calcd for $\mathrm{C}_{22} \mathrm{H}_{48} \mathrm{NO}_{3} \mathrm{Si}_{2}, 430.3173$; found, 430.3170 .

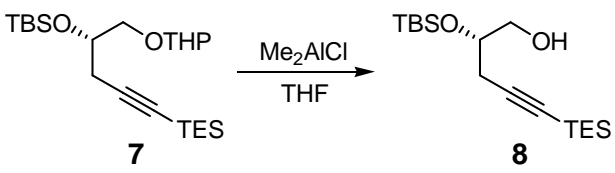




\section{Alcohol 8}

A solution of dimethylaluminum chloride in hexanes $(1.0 \mathrm{M}, 125 \mathrm{~mL}, 125 \mathrm{mmol}, 2.5$ equiv) was added dropwise via cannula over $20 \mathrm{~min}$ to a solution of the tert-butyldimethylsilyl ether 7 $\left(20.7 \mathrm{~g}, 50.1 \mathrm{mmol}, 1.0\right.$ equiv) in dichloromethane $(300 \mathrm{~mL})$ at $-78^{\circ} \mathrm{C}$. The resulting mixture was allowed to warm to $23{ }^{\circ} \mathrm{C}$ over $2 \mathrm{~h}$. The reaction mixture was then transferred by cannula into a mixture of saturated aqueous potassium sodium tartrate solution $(300 \mathrm{~mL})$ and saturated aqueous sodium bicarbonate solution $(300 \mathrm{~mL})$. The resulting biphasic solution was stirred at $23{ }^{\circ} \mathrm{C}$ for $1 \mathrm{~h}$. The layers were separated. The aqueous layer was extracted with dichloromethane $(2 \times 400 \mathrm{~mL})$. The organic extracts were combined and the combined solution was dried over anhydrous sodium sulfate. The dried solution was filtered and the filtrate was concentrated. The residue was purified by flash-column chromatography (17\% ether-hexanes) to provide the alcohol $\mathbf{8}$ (colorless oil, $15.28 \mathrm{~g}, 93 \%)$.

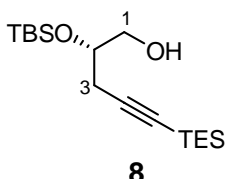

$\mathrm{R}_{f}=0.40$ (20\% ether-hexanes). ${ }^{1} \mathrm{H}$ NMR (400 MHz, $\left.\mathrm{CDCl}_{3}\right), \delta 3.91-3.86(\mathrm{~m}, 1 \mathrm{H}, \mathrm{C}(2)-\mathbf{H})$, 3.71-3.66 (m, 1H, C(1)-H), 3.63-3.57 (m, 1H, C(1)-H), $2.48\left(\mathrm{dd}, \mathrm{J}_{1}=7.6 \mathrm{~Hz}, \mathrm{~J}_{2}=16.8 \mathrm{~Hz}, 1 \mathrm{H}\right.$, $\mathrm{C}(3)-\mathbf{H}), 2.41\left(\mathrm{dd}, \mathrm{J}_{1}=5.2 \mathrm{~Hz}, \mathrm{~J}_{2}=16.8 \mathrm{~Hz}, 1 \mathrm{H}, \mathrm{C}(3)-\mathbf{H}\right), 1.91\left(\mathrm{dd}, \mathrm{J}_{1}=6.8 \mathrm{~Hz}, \mathrm{~J}_{2}=7.2,1 \mathrm{H}\right.$, $\mathrm{C}(1)-\mathrm{OH}), 0.97$ (t, J = 7.6 Hz, 9H, Si( $\left.\left.\mathrm{CH}_{2} \mathrm{CH}_{3}\right)_{3}\right), 0.89$ (s, 9H, Si( $\left.\left(\mathrm{CH}_{3}\right)_{2} \mathrm{C}\left(\mathrm{CH}_{3}\right)_{3}\right), 0.56$ (q, J = 7.6 $\left.\mathrm{Hz}, 6 \mathrm{H}, \mathrm{Si}\left(\mathrm{CH}_{2} \mathrm{CH}_{3}\right)_{3}\right), 0.12$ (s, 3H, $\left.\mathrm{Si}\left(\mathrm{CH}_{3}\right)_{2} \mathrm{C}\left(\mathrm{CH}_{3}\right)_{3}\right), 0.10$ (s, 3H, Si $\left.\left(\mathrm{CH}_{3}\right)_{2} \mathrm{C}\left(\mathrm{CH}_{3}\right)_{3}\right) .{ }^{13} \mathrm{C} \mathrm{NMR}$ $\left(100 \mathrm{MHz}, \mathrm{CDCl}_{3}\right), \delta$ 104.0, 84.0, 72.8, 71.5, 65.7, 63.8, 44.6, 25.7 (2), 25.6, 25.4, 18.0, 7.4, 4.4, $-3.6,-4.6,-4.7,-4.8$. IR ( $\mathrm{NaCl}$, thin film) $\mathrm{cm}^{-1} 3421,2955,2931,2876,2858,2175,1472,1463$, $1415,1389,1363,1255,1115,1047,1020,1005,975,938,854,838,808,778$. HRMS-ESI $(\mathrm{m} / \mathrm{z})$ $[\mathrm{M}+\mathrm{H}]^{+}$calcd for $\mathrm{C}_{17} \mathrm{H}_{37} \mathrm{O}_{2} \mathrm{Si}_{2}, 329.2332$; found, 329.2331 .

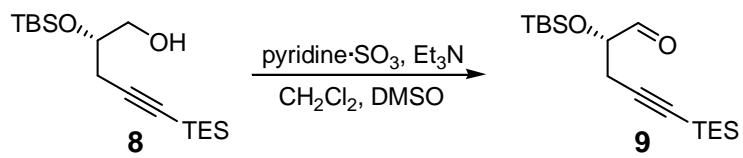

\section{Aldehyde 9}

A freshly-prepared solution of sulfur trioxide-pyridine complex $(7.27 \mathrm{~g}, 45.7 \mathrm{mmol}, 5.0$ equiv) in DMSO $(50 \mathrm{~mL})$ was added dropwise via cannula over $10 \mathrm{~min}$ to a stirring solution of the alcohol 8 (3.00 g, $9.13 \mathrm{mmol}, 1$ equiv) and triethylamine (12.7 mL, $91.3 \mathrm{mmol}, 10$ equiv) in dichloromethane $(60 \mathrm{~mL})$ at $0{ }^{\circ} \mathrm{C}$. After stirring at $0{ }^{\circ} \mathrm{C}$ for $2 \mathrm{~h}$, the reaction mixture was diluted with a 1:1 mixture of ethyl acetate and hexanes $(400 \mathrm{~mL})$. The resulting mixture was washed successively with $1 \mathrm{~N}$ aqueous hydrochloric acid solution $(2 \times 60 \mathrm{~mL})$, saturated aqueous sodium bicarbonate solution $(80 \mathrm{~mL})$, and brine $(80 \mathrm{~mL})$. The washed organic solution was dried over anhydrous sodium sulfate. The dried solution was filtered and the filtrate was concentrated to give the aldehyde 9 (colorless oil, $2.93 \mathrm{~g}, 98 \%$ ), used in the next reaction without purification.

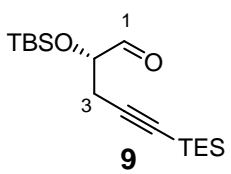


$\mathrm{R}_{f}=0.43$ (17\% ether-hexanes). ${ }^{1} \mathrm{H}$ NMR (400 MHz, $\left.\mathrm{CDCl}_{3}\right), \delta 9.64(\mathrm{~d}, \mathrm{~J}=1.2 \mathrm{~Hz}, 1 \mathrm{H}, \mathrm{C}(1)-\mathbf{H})$, $4.10\left(\mathrm{dt}, \mathrm{J}_{1}=1.2 \mathrm{~Hz}, \mathrm{~J}_{2}=6.2 \mathrm{~Hz}, 1 \mathrm{H}, \mathrm{C}(2)-\mathbf{H}\right), 2.65\left(\mathrm{dd}, \mathrm{J}_{1}=5.6 \mathrm{~Hz}, \mathrm{~J}_{2}=17.2 \mathrm{~Hz}, 1 \mathrm{H}, \mathrm{C}(3)-\mathbf{H}\right)$, $2.55\left(\mathrm{dd}, \mathrm{J}_{1}=7.2 \mathrm{~Hz}, \mathrm{~J}_{2}=17.2 \mathrm{~Hz}, 1 \mathrm{H}, \mathrm{C}(3)-\mathrm{H}\right), 0.96\left(\mathrm{t}, \mathrm{J}=7.6 \mathrm{~Hz}, 9 \mathrm{H}, \mathrm{Si}\left(\mathrm{CH}_{2} \mathrm{CH}_{3}\right)_{3}\right), 0.92(\mathrm{~s}, 9 \mathrm{H}$, $\left.\mathrm{Si}\left(\mathrm{CH}_{3}\right)_{2} \mathrm{C}\left(\mathrm{CH}_{3}\right)_{3}\right), 0.56$ (q, J = 7.6 Hz, 6H, Si $\left.\left(\mathrm{CH}_{2} \mathrm{CH}_{3}\right)_{3}\right), 0.13$ (s, 3H, $\left.\mathrm{Si}\left(\mathrm{CH}_{3}\right)_{2} \mathrm{C}\left(\mathrm{CH}_{3}\right)_{3}\right), 0.12$ (s, $\left.3 \mathrm{H}, \mathrm{Si}\left(\mathrm{CH}_{3}\right)_{2} \mathrm{C}\left(\mathrm{CH}_{3}\right)_{3}\right) .{ }^{13} \mathrm{C} \mathrm{NMR}\left(100 \mathrm{MHz}, \mathrm{CDCl}_{3}\right), \delta 202.2,102.5,84.8,76.0,25.7,24.6,18.2$, 7.4, 4.3, -4.8 (2). IR ( $\mathrm{NaCl}$, thin film), $\mathrm{cm}^{-1}$ 2950, 2934, 2876, 2806, 2178, 1742, 1464, 1416, $1377,1362,1334,1255,1124,1019,1006,972,937,839,780,738 . \quad$ HRMS-CI $(\mathrm{m} / \mathrm{z})\left[\mathrm{M}+\mathrm{NH}_{4}\right]^{+}$ calcd for $\mathrm{C}_{17} \mathrm{H}_{38} \mathrm{NO}_{2} \mathrm{Si}_{2}, 344.2441$; found, 344.2435.

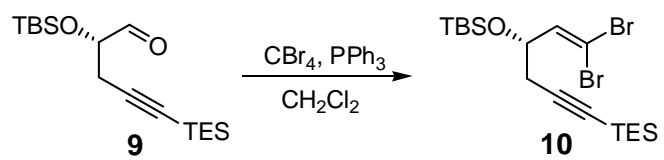

\section{Dibromoolefin 10}

Triphenylphosphine $(9.44 \mathrm{~g}, 36.0 \mathrm{mmol}, 4.0$ equiv) was added to a solution of carbon tetrabromide $\left(5.97 \mathrm{~g}, 18.0 \mathrm{mmol}, 2.0\right.$ equiv) in dichloromethane $(60 \mathrm{~mL})$ at $0{ }^{\circ} \mathrm{C}$. After $15 \mathrm{~min}$, a solution of the aldehyde 9 ( $2.93 \mathrm{~g}, 9.00 \mathrm{mmol}, 1$ equiv) in dichloromethane $(40 \mathrm{~mL})$ was added dropwise via cannula over $30 \mathrm{~min}$ at $0{ }^{\circ} \mathrm{C}$. After stirring at $0{ }^{\circ} \mathrm{C}$ for $45 \mathrm{~min}$, the reaction suspension was diluted with a 1:4 mixture of ether and pentane $(300 \mathrm{~mL})$. The mixture was filtered through a short plug of silica gel. The filter cake was washed with a 1:4 mixture of ether and pentane $(300 \mathrm{~mL})$. The filtrates were combined and the combined solution was concentrated. The residue was purified by flash-column chromatography (100\% hexanes) to provide the dibromoolefin 10 (yellow oil, 95\% ee, $3.32 \mathrm{~g}, 77 \%, 2$ steps). The enantiomeric excess of $\mathbf{1 0}$ was determined by Mosher ester analysis of a derivative, prepared as shown in the scheme below.
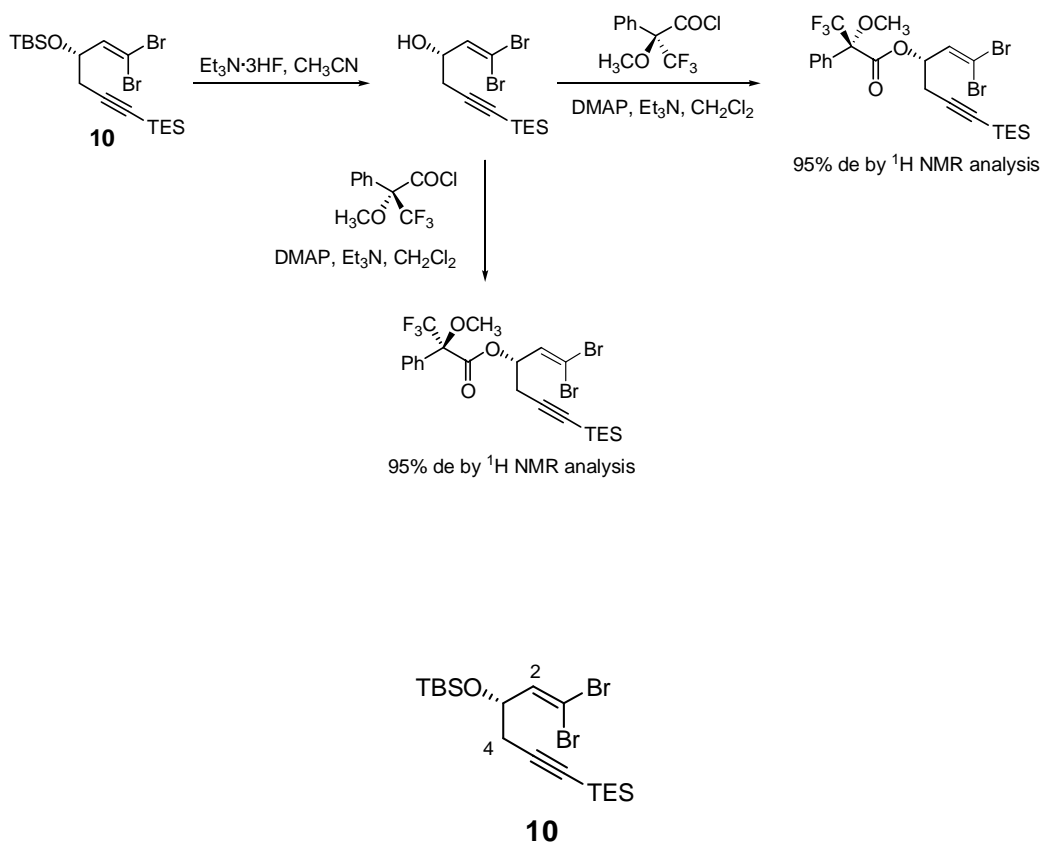

$\mathrm{R}_{f}=0.26$ (100\% hexanes). $\quad{ }^{1} \mathrm{H}$ NMR (500 MHz, $\left.\mathrm{CDCl}_{3}\right), \delta 6.43(\mathrm{~d}, \mathrm{~J}=8.0 \mathrm{~Hz}, 1 \mathrm{H}, \mathrm{C}(2)-\mathbf{H}), 4.47$ $\left(\mathrm{dt}, \mathrm{J}_{1}=6.5 \mathrm{~Hz}, \mathrm{~J}_{2}=8.0 \mathrm{~Hz}, 1 \mathrm{H}, \mathrm{C}(3)-\mathbf{H}\right), 2.50\left(\mathrm{dd}, \mathrm{J}_{1}=6.0 \mathrm{~Hz}, \mathrm{~J}_{2}=17.0 \mathrm{~Hz}, 1 \mathrm{H}, \mathrm{C}(4)-\mathbf{H}\right), 2.45$ (dd, 
$\left.\mathrm{J}_{1}=6.0 \mathrm{~Hz}, \mathrm{~J}_{2}=17.0 \mathrm{~Hz}, 1 \mathrm{H}, \mathrm{C}(4)-\mathbf{H}\right), 0.99\left(\mathrm{t}, \mathrm{J}=8.0 \mathrm{~Hz}, 9 \mathrm{H}, \mathrm{Si}\left(\mathrm{CH}_{2} \mathrm{CH}_{3}\right)_{3}\right), 0.89(\mathrm{~s}, 9 \mathrm{H}$, $\left.\mathrm{Si}\left(\mathrm{CH}_{3}\right)_{2} \mathrm{C}\left(\mathrm{CH}_{3}\right)_{3}\right), 0.59$ (q, J = 8.0 Hz, 6H, Si( $\left.\left(\mathrm{CH}_{2} \mathrm{CH}_{3}\right)_{3}\right), 0.11$ (s, 3H, $\left.\mathrm{Si}\left(\mathrm{CH}_{3}\right)_{2} \mathrm{C}\left(\mathrm{CH}_{3}\right)_{3}\right), 0.09$ (s, $\left.3 \mathrm{H}, \mathrm{Si}\left(\mathrm{CH}_{3}\right)_{2} \mathrm{C}\left(\mathrm{CH}_{3}\right)_{3}\right) .{ }^{13} \mathrm{C} \mathrm{NMR}\left(100 \mathrm{MHz}, \mathrm{CDCl}_{3}\right), \delta$ 140.4, 102.9, 90.1, 84.3, 72.1, 28.5, 25.7, 18.0, 7.5, 4.4, -4.6, -4.9. IR ( $\mathrm{NaCl}$, thin film) $\mathrm{cm}^{-1} 3427,2955,2931,2898,2875,2858,2178$, $1623,1472,1462,1414,1362,1253,1086,1018,931,836,778,726 . \quad[\alpha]^{23}{ }_{D}-9.6^{\circ}\left(\mathrm{c} 0.57, \mathrm{CHCl}_{3}\right)$. HRMS-ESI $(m / z)[\mathrm{M}+\mathrm{H}]^{+}$calcd for $\mathrm{C}_{17} \mathrm{H}_{35} \mathrm{O}_{2} \mathrm{Si}_{2}, 481.0593$; found, 481.0588 .

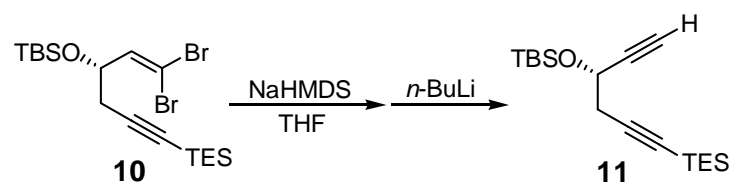

\section{Diyne 11}

A solution of sodium bis(trimethylsilyl)amide in tetrahydrofuran (1.0 M, $11.2 \mathrm{~mL}, 11.2$ mmol, 1.2 equiv) was added dropwise via syringe over $10 \mathrm{~min}$ to a solution of the dibromoolefin $\mathbf{1 0}$ ( $4.51 \mathrm{~g}, 9.35 \mathrm{mmol}, 1$ equiv) in tetrahydrofuran $(50 \mathrm{~mL})$ at $-78{ }^{\circ} \mathrm{C}$. After $20 \mathrm{~min}$, a solution of $n$-butyllithium in hexanes $(2.50 \mathrm{M}, 7.48 \mathrm{~mL}, 18.7 \mathrm{mmol}, 2.0$ equiv) was added dropwise via syringe over $15 \mathrm{~min}$. The reaction mixture was stirred for an additional $10 \mathrm{~min}$ at $-78{ }^{\circ} \mathrm{C}$. Saturated aqueous ammonium chloride solution $(80 \mathrm{~mL})$ was then added to quench any excess base. The resulting biphasic mixture was partitioned between ether $(150 \mathrm{~mL})$ and water $(50 \mathrm{~mL})$. The layers were separated, and the aqueous layer was extracted with ether $(2 \times 80 \mathrm{~mL})$. The organic extracts were combined and the combined solution was washed with brine $(80 \mathrm{~mL})$. The washed organic solution was dried over anhydrous sodium sulfate. The dried solution was filtered and the filtrate was concentrated. The residue was purified by flash-column chromatography ( $2 \%$ ether-hexanes) to provide the diyne $\mathbf{1 1}$ (colorless oil, $2.99 \mathrm{~g}, 99 \%$ ).

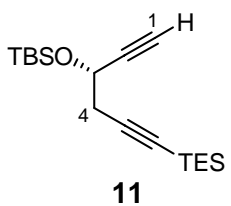

$\mathrm{R}_{f}=0.30$ (100\% hexanes). ${ }^{1} \mathrm{H}$ NMR $\left(400 \mathrm{MHz}, \mathrm{CDCl}_{3}\right), \delta 4.48\left(\mathrm{dt}, \mathrm{J}_{1}=2.4 \mathrm{~Hz}, \mathrm{~J}_{2}=6.8 \mathrm{~Hz}, 1 \mathrm{H}\right.$, C(3)-H), 2.63 (d, J = 6.8 Hz, 2H, C(4)-H), 2.40 (d, J = 2.4 Hz, 1H, C(1)-H), 0.98 (t, J = 8.0 Hz, 9H, $\left.\mathrm{Si}\left(\mathrm{CH}_{2} \mathrm{CH}_{3}\right)_{3}\right), 0.91$ (s, 9H, $\left.\mathrm{Si}\left(\mathrm{CH}_{3}\right)_{2} \mathrm{C}\left(\mathrm{CH}_{3}\right)_{3}\right), 0.57$ (q, J = 8.0 Hz, 6H, $\left.\mathrm{Si}\left(\mathrm{CH}_{2} \mathrm{CH}_{3}\right)_{3}\right), 0.15$ (s, 3H, $\left.\mathrm{Si}\left(\mathrm{CH}_{3}\right)_{2} \mathrm{C}\left(\mathrm{CH}_{3}\right)_{3}\right), 0.14\left(\mathrm{~s}, 3 \mathrm{H}, \mathrm{Si}\left(\mathrm{CH}_{3}\right)_{2} \mathrm{C}\left(\mathrm{CH}_{3}\right)_{3}\right) .{ }^{13} \mathrm{C} \mathrm{NMR}\left(100 \mathrm{MHz}, \mathrm{CDCl}_{3}\right), \delta$ 103.7, 84.3, 84.0, 72.5, 62.1, 30.6, 25.7, 18.2, 7.4, 4.4, -4.7, -5.0. IR (NaCl, thin film), $\mathrm{cm}^{-1} 3311,2956,2935$, 2913, 2876, 2858, 2179, 1644, 1471, 1463, 1415, 1396, 1362, 1345, 1253, 1100, 1013, 950, 930, 839, 779. $[\alpha]^{23}-13.9^{\circ}\left(\mathrm{c} 0.61, \mathrm{CHCl}_{3}\right)$. HRMS-ESI $(\mathrm{m} / \mathrm{z})\left[\mathrm{M}+\mathrm{NH}_{4}\right]^{+}$calcd for $\mathrm{C}_{18} \mathrm{H}_{38} \mathrm{NOSi}_{2}$, 340.2492; found, 340.2495 .

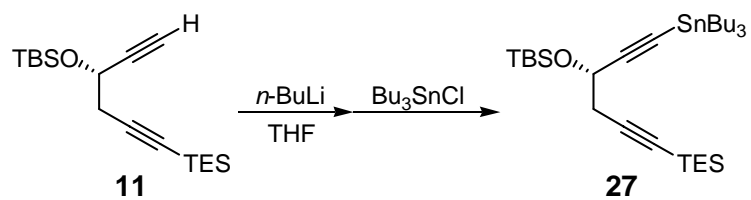

Tributylstannanyl Alkyne 27

A solution of $n$-butyllithium in hexanes $(2.50 \mathrm{M}, 3.22 \mathrm{~mL}, 8.05 \mathrm{mmol}, 1.1$ equiv) was 
added dropwise via syringe over $15 \mathrm{~min}$ to a solution of the diyne $\mathbf{1 1}$ ( $2.36 \mathrm{~g}, 7.31 \mathrm{mmol}, 1$ equiv) in tetrahydrofuran $(30 \mathrm{~mL})$ at $-78{ }^{\circ} \mathrm{C}$. After $15 \mathrm{~min}$, tributyltin chloride $(2.08 \mathrm{~mL}, 7.68 \mathrm{mmol}$, 1.05 equiv) was added via syringe over $2 \mathrm{~min}$. The reaction mixture was allowed to warm to 23 ${ }^{\circ} \mathrm{C}$ over $1 \mathrm{~h}$. Water $(80 \mathrm{~mL})$ was added and tetrahydrofuran was removed in vacuo. The biphasic residue was extracted with ether $(300 \mathrm{~mL})$. The organic layer was separated and washed with water $(2 \times 80 \mathrm{~mL})$. The washed organic solution was dried over anhydrous sodium sulfate. The dried solution was filtered and the filtrate was concentrated to provide the tributylstannanyl alkyne 27 (colorless oil, $4.47 \mathrm{~g}, 100 \%$ ), used in next reaction without purification.

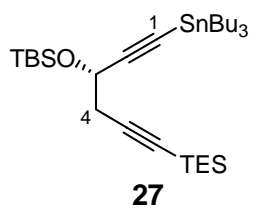

${ }^{1} \mathrm{H}$ NMR $\left(500 \mathrm{MHz}, \mathrm{CDCl}_{3}\right), \delta 4.48(\mathrm{t}, \mathrm{J}=6.5 \mathrm{~Hz}, 1 \mathrm{H}, \mathrm{C}(3)-\mathbf{H}), 2.61$ (d, J = 6.5 Hz, 2H, C(4)-H), 1.54 (m, 6H, $\mathrm{Sn}\left(\mathrm{CH}_{2} \mathrm{CH}_{2} \mathrm{CH}_{2} \mathrm{CH}_{3}\right)_{3}, 1.33$ (m, 6H, Sn( $\left.\left(\mathrm{CH}_{2} \mathrm{CH}_{2} \mathrm{CH}_{2} \mathrm{CH}_{3}\right)_{3}\right), 0.98$ (t, J = 8.0 Hz, 9H, $\left.\mathrm{Si}\left(\mathrm{CH}_{2} \mathrm{CH}_{3}\right)_{3}\right), 0.96\left(\mathrm{~m}, 6 \mathrm{H}, \mathrm{Sn}\left(\mathrm{CH}_{2} \mathrm{CH}_{2} \mathrm{CH}_{2} \mathrm{CH}_{3}\right)_{3}, 0.91\right.$ (s, 9H, $\left.\mathrm{Si}\left(\mathrm{CH}_{3}\right)_{2} \mathrm{C}\left(\mathrm{CH}_{3}\right)_{3}\right), 0.90$ (t, J = 7.5 $\left.\mathrm{Hz}, 9 \mathrm{H}, \mathrm{Sn}\left(\mathrm{CH}_{2} \mathrm{CH}_{2} \mathrm{CH}_{2} \mathrm{CH}_{3}\right)_{3}\right), 0.57\left(\mathrm{q}, \mathrm{J}=8.0 \mathrm{~Hz}, 6 \mathrm{H}, \mathrm{Si}\left(\mathrm{CH}_{2} \mathrm{CH}_{3}\right)_{3}\right), 0.16$ (s, 3H, $\left.\mathrm{Si}\left(\mathrm{CH}_{3}\right)_{2} \mathrm{C}\left(\mathrm{CH}_{3}\right)_{3}\right), 0.14$ (s, 3H, $\left.\mathrm{Si}\left(\mathrm{CH}_{3}\right)_{2} \mathrm{C}\left(\mathrm{CH}_{3}\right)_{3}\right)$.

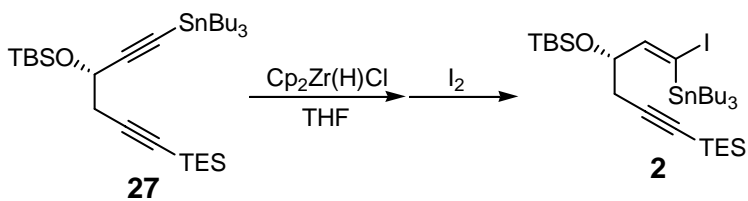

\section{(1-Iodovinyl) Stannane 2}

A solution of the tributylstannanyl alkyne 27 (4.47 g, $7.31 \mathrm{mmol}, 1$ equiv) in tetrahydrofuran $(50 \mathrm{~mL})$ was added dropwise via cannula over 10 min to a suspension of bis(cyclopentadienyl) zirconium chloride hydride (Schwartz's reagent, Strem Chemicals, $2.64 \mathrm{~g}$, $10.2 \mathrm{mmol}, 1.4$ equiv) in tetrahydrofuran $(60 \mathrm{~mL})$ at $23{ }^{\circ} \mathrm{C}$. After stirring at $23{ }^{\circ} \mathrm{C}$ for $40 \mathrm{~min}$, the reaction solution had become dark-red. A solution of iodine (1.95 g, $7.68 \mathrm{mmol}, 1.05$ equiv) in tetrahydrofuran $(15 \mathrm{~mL})$ was added dropwise via cannula over $5 \mathrm{~min}$. After stirring at $23{ }^{\circ} \mathrm{C}$ for $20 \mathrm{~min}$, the resulting light-yellow solution was partitioned between ether $(300 \mathrm{~mL})$ and water $(150$ $\mathrm{mL})$. The organic layer was separated and washed with brine $(60 \mathrm{~mL})$. The washed organic solution was dried over anhydrous sodium sulfate. The dried solution was filtered and the filtrate was concentrated. The residue was purified by flash-column chromatography (100\% hexanes, silica gel deactivated with $\mathrm{Et}_{3} \mathrm{~N}$ ) to provide the (1-iodovinyl) stannane 2 (yellowish oil, 3.71g, 69\%, 2 steps).

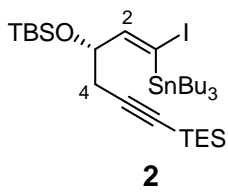

$\mathrm{R}_{f}=0.25$ (100\% hexanes). ${ }^{1} \mathrm{H}$ NMR (500MHz, $\left.\mathrm{CDCl}_{3}\right), \delta 7.31$ (ddt, $\mathrm{J}_{1}=2.5 \mathrm{~Hz}, \mathrm{~J}_{2}=9.0 \mathrm{~Hz}, \mathrm{~J}_{3}=$ $49.75 \mathrm{~Hz}, 1 \mathrm{H}, \mathrm{C}(2)-\mathbf{H}), 4.04\left(\mathrm{dt}, \mathrm{J}_{1}=4.5 \mathrm{~Hz}, \mathrm{~J}_{2}=8.5 \mathrm{~Hz}, 1 \mathrm{H}, \mathrm{C}(3)-\mathbf{H}\right), 2.45\left(\mathrm{dd}, \mathrm{J}_{1}=8.0 \mathrm{~Hz}, \mathrm{~J}_{2}=\right.$ $17.0 \mathrm{~Hz}, 1 \mathrm{H}, \mathrm{C}(4)-\mathbf{H}), 2.33\left(\mathrm{dd}, \mathrm{J}_{1}=4.5 \mathrm{~Hz}, \mathrm{~J}_{2}=17.0 \mathrm{~Hz}, 1 \mathrm{H}, \mathrm{C}(4)-\mathbf{H}\right), 1.57-1.61(\mathrm{~m}, 6 \mathrm{H}$, $\mathrm{Sn}\left(\mathrm{CH}_{2} \mathrm{CH}_{2} \mathrm{CH}_{2} \mathrm{CH}_{3}\right)_{3}, 1.35\left(\mathrm{~m}, 6 \mathrm{H}, \mathrm{Sn}\left(\mathrm{CH}_{2} \mathrm{CH}_{2} \mathrm{CH}_{2} \mathrm{CH}_{3}\right)_{3}\right), 1.09$ (m, 6H, $\mathrm{Sn}\left(\mathrm{CH}_{2} \mathrm{CH}_{2} \mathrm{CH}_{2} \mathrm{CH}_{3}\right)_{3}$, 
$0.98\left(\mathrm{t}, \mathrm{J}=8.0 \mathrm{~Hz}, 9 \mathrm{H}, \mathrm{Si}\left(\mathrm{CH}_{2} \mathrm{CH}_{3}\right)_{3}\right), 0.92\left(\mathrm{t}, \mathrm{J}=7.0 \mathrm{~Hz}, 9 \mathrm{H}, \mathrm{Sn}\left(\mathrm{CH}_{2} \mathrm{CH}_{2} \mathrm{CH}_{2} \mathrm{CH}_{3}\right)_{3}\right), 0.88$ (s, 9H, $\left.\mathrm{Si}\left(\mathrm{CH}_{3}\right)_{2} \mathrm{C}\left(\mathrm{CH}_{3}\right)_{3}\right), 0.57$ (q, J = 8.0 Hz, 6H, Si( $\left.\left.\mathrm{CH}_{2} \mathrm{CH}_{3}\right)_{3}\right), 0.09$ (s, 3H, $\left.\mathrm{Si}\left(\mathrm{CH}_{3}\right)_{2} \mathrm{C}\left(\mathrm{CH}_{3}\right)_{3}\right), 0.07$ (s, $\left.3 \mathrm{H}, \mathrm{Si}\left(\mathrm{CH}_{3}\right)_{2} \mathrm{C}\left(\mathrm{CH}_{3}\right)_{3}\right) .{ }^{13} \mathrm{C} \mathrm{NMR}\left(100 \mathrm{MHz}, \mathrm{CDCl}_{3}\right), \delta$ 158.6, 104.2, 103.4, 83.5, $75.6(\mathrm{t}, \mathrm{J}=11.4$ $\mathrm{Hz}$ ), 7.5, 4.4, -4.1, -4.4. IR ( $\mathrm{NaCl}$, thin film) $\mathrm{cm}^{-1}$ 2956, 2930, 2874, 2856, 2176, 1403, 1251, $1098,837,777,738,726 .[\alpha]^{23}-12.1^{\circ}\left(\mathrm{c} 1.2, \mathrm{CHCl}_{3}\right)$. HRMS-ESI $(\mathrm{m} / \mathrm{z})\left[\mathrm{M}-\mathrm{C}_{4} \mathrm{H}_{9}\right]^{+}$calcd for $\mathrm{C}_{26} \mathrm{H}_{52} \mathrm{IOSi}_{2} \mathrm{Sn}, 683.1623$; found, 683.1636.
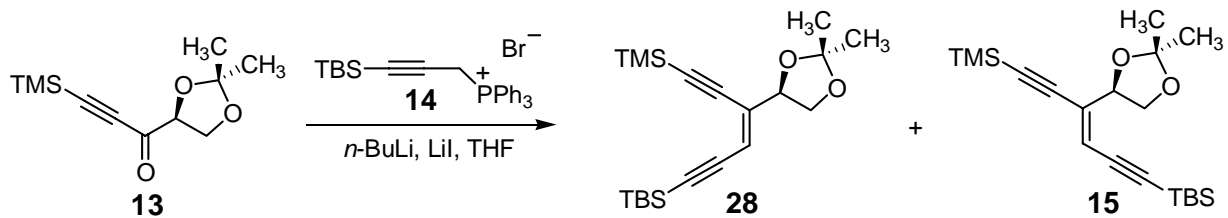

\section{$\underline{(Z)-O l e f i n} 15$}

A solution of $n$-butyllithium in hexanes $(2.50 \mathrm{M}, 9.64 \mathrm{~mL}, 24.1 \mathrm{mmol}, 1.2$ equiv) was added dropwise via syringe over $15 \mathrm{~min}$ to a thoroughly degassed, stirring suspension of 3-(tert-butyldimethylsilyl)propargyltriphenylphosphonium bromide ${ }^{5}$ (14, 13.0 g, $26.3 \mathrm{mmol}, 1.3$ equiv) and lithium iodide $\left(12.1 \mathrm{~g}, 90.6 \mathrm{mmol}, 4.6\right.$ equiv) in tetrahydrofuran $(250 \mathrm{~mL})$ at $-78{ }^{\circ} \mathrm{C}$. After the addition was complete, the reaction mixture was warmed to $0{ }^{\circ} \mathrm{C}$ in an ice-water bath. After stirring at $0{ }^{\circ} \mathrm{C}$ for $20 \mathrm{~min}$, the reaction mixture was cooled to $-78{ }^{\circ} \mathrm{C}$. A solution of the propargylic ketone $\mathbf{1 3}^{5,6}$ (4.46 g, $19.7 \mathrm{mmol}, 1$ equiv) in tetrahydrofuran $(100 \mathrm{~mL}$ ) was added dropwise via cannula over $15 \mathrm{~min}$. The reaction mixture was stirred at $-78^{\circ} \mathrm{C}$ for $1 \mathrm{~h}$. Saturated aqueous ammonium chloride solution $(150 \mathrm{~mL})$ was added to quench any excess base. The layers were separated and the aqueous layer was extracted successively with pentane $(2 \times 150 \mathrm{~mL})$ and ether $(2 \times 150 \mathrm{~mL})$. The organic extractions were combined and the combined solution was washed with brine $(3 \times 100 \mathrm{~mL})$. The washed organic solution was dried over anhydrous sodium sulfate. The dried solution was filtered and the filtrate was concentrated. The residue was purified by flash-column chromatography (5\% ether-hexanes) to provide the $(Z)$-olefin 15 (yellow oil, $4.45 \mathrm{~g}, 74 \%$ ) and, separately, the (E)-olefin $\mathbf{2 8}$ (yellow oil, $0.92 \mathrm{~g}, 15 \%$ ).

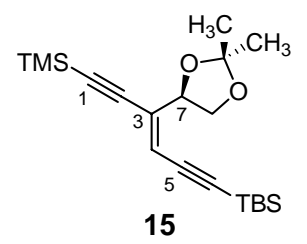

$\mathrm{R}_{f}(\mathbf{1 5})=0.53, \mathrm{R}_{f}(\mathbf{2 8})=0.45$ (10\% ether-hexanes). ${ }^{1} \mathrm{H} \mathrm{NMR}\left(500 \mathrm{MHz}, \mathrm{C}_{6} \mathrm{D}_{6}\right), \delta 6.01(\mathrm{~s}, 1 \mathrm{H}$, $\mathrm{C}(4)-\mathbf{H}), 5.49$ (t, J = 7.5 Hz, 1H, C(7)-H), 4.09 (dd, $\left.\mathrm{J}_{1}=7.5 \mathrm{~Hz}, \mathrm{~J}_{2}=8.0 \mathrm{~Hz}, 1 \mathrm{H}, \mathrm{C}(8)-\mathbf{H}\right), 3.96\left(\mathrm{t}, \mathrm{J}_{1}\right.$ $\left.=7.5 \mathrm{~Hz}, \mathrm{~J}_{2}=8.0 \mathrm{~Hz}, 1 \mathrm{H}, \mathrm{C}(8)-\mathbf{H}\right), 1.57\left(\mathrm{~s}, 3 \mathrm{H}, \mathrm{C}\left(\mathrm{CH}_{3}\right)_{2}\right), 1.39\left(\mathrm{~s}, 3 \mathrm{H}, \mathrm{C}\left(\mathrm{CH}_{3}\right)_{2}\right), 0.93(\mathrm{~s}, 9 \mathrm{H}$, $\left.\mathrm{SiC}\left(\mathrm{CH}_{3}\right)_{3}\left(\mathrm{CH}_{3}\right)_{2}\right), 0.14$ (s, 9H, $\left.\mathrm{Si}\left(\mathrm{CH}_{3}\right)_{3}\right), 0.05$ (s, 3H, $\left.\mathrm{SiC}\left(\mathrm{CH}_{3}\right)_{3}\left(\mathrm{CH}_{3}\right)_{2}\right), 0.04 \quad$ (s, 3H, $\left.\mathrm{SiC}\left(\mathrm{CH}_{3}\right)_{3}\left(\mathrm{CH}_{3}\right)_{2}\right) .{ }^{13} \mathrm{C} \mathrm{NMR}\left(100 \mathrm{MHz}, \mathrm{C}_{6} \mathrm{D}_{6}\right), \delta$ 136.2, 118.8, 110.4, 105.7, 103.0, 101.9, 101.5, $75.2,68.4,26.7,26.3,26.1,16.8,-0.3,-4.8$. IR ( $\mathrm{NaCl}$, thin film), $\mathrm{cm}^{-1} 2955,2930,2858,2144$,

\footnotetext{
5 (a) Myers, A. G.; Hammond, M.; Wu, Y.; Xiang, J.-N.; Harrington, P. M.; Kuo, E. Y. J. Am. Chem. Soc. 1996, 118, 10006. (b) Hammond, M., Ph. D. Thesis, California Institute of Technology, 1997.

${ }^{6}$ For the synthesis of $(S)$-glyceraldehyde acetonide, see: (a) Janson, M.; Kvarnström, I.; Svensson, S. C. T.; Classon, B.; Samuelsson, B. Synthesis 1993, 129. (b) Hubschwerlen, C. Synthesis 1986, 962. (c) Schmid, C. R.; Bradley, D. A. Synthesis 1992, 587.
} 
1472, 1371, 1251, 1067, 841, 776. HRMS-ESI $(\mathrm{m} / \mathrm{z})[\mathrm{M}+\mathrm{H}]^{+}$calcd for $\mathrm{C}_{20} \mathrm{H}_{35} \mathrm{O}_{2} \mathrm{Si}_{2}, 363.2175$; found, 363.2167.

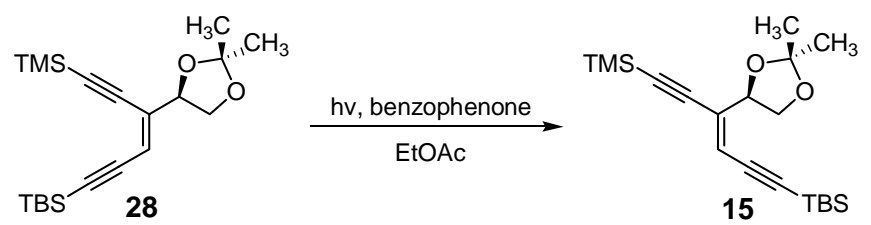

\section{Conversion of the $(E)$-Olefin 28 to the $(Z)$-Olefin 15}

A 100-mL, round-bottom flask was charged with the $(E)$-olefin 28 (2.35 g, $6.47 \mathrm{mmol}, 1$ equiv), benzophenone (226 mg, $1.24 \mathrm{mmol}, 0.2$ equiv), and ethyl acetate (15 mL). The reaction vessel was irradiated with a Hanovia 450-watt medium-pressure mercury lamp (Pyrex filter) for $4 \mathrm{~h}$. ${ }^{1} \mathrm{H}$ NMR analysis of the reaction mixture at this point showed that a 55:45 mixture of the $(E)$-olefin $\mathbf{2 8}$ and the (Z)-olefin $\mathbf{1 5}$ had formed. The reaction mixture was concentrated and the isomers were separated by flash-column chromatography (5\% ether-hexanes) to provide the (Z)-olefin $\mathbf{1 5}$ (yellow oil, $0.94 \mathrm{~g}, 40 \%$ ) and, separately, recovered $(E)$-olefin 28 (yellow oil, $1.37 \mathrm{~g}, 58 \%$ ). This process was subject to iteration.

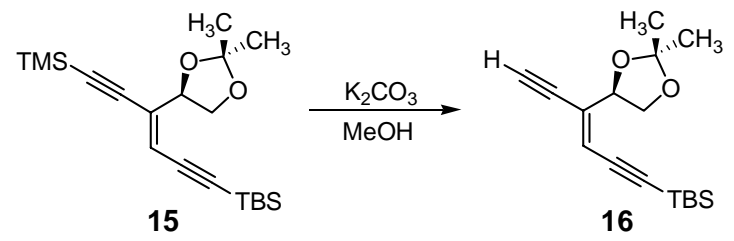

\section{Enediyne 16}

Potassium carbonate ( $336 \mathrm{mg}, 2.43 \mathrm{mmol}, 1$ equiv) was added to a solution of the (Z)-olefin 15 (882 mg, $2.43 \mathrm{mmol}, 1$ equiv) in methanol $(17 \mathrm{~mL})$ at $0{ }^{\circ} \mathrm{C}$. After stirring at $0{ }^{\circ} \mathrm{C}$ for $45 \mathrm{~min}$, the reaction mixture was partitioned between pentane $(50 \mathrm{~mL})$ and water $(15 \mathrm{~mL})$. The aqueous layer was separated and extracted with pentane $(2 \times 20 \mathrm{~mL})$. The organic extracts were combined and the combined solution was dried over anhydrous sodium sulfate. The dried organic solution was filtered and the filtrate was concentrated. The residue was purified by flash-column chromatography (10\% ether-hexanes) to give the enediyne $\mathbf{1 6}$ (yellow oil, $705 \mathrm{mg}, 100 \%$ ).

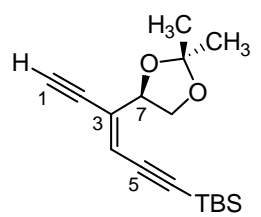

16

$\mathrm{R}_{f}=0.39$ (10\% ether-hexanes). ${ }^{1} \mathrm{H}$ NMR (500 MHz, $\left.\mathrm{C}_{6} \mathrm{D}_{6}\right), \delta 5.96(\mathrm{~s}, 1 \mathrm{H}, \mathrm{C}(4)-\mathrm{H}), 5.45(\mathrm{t}, \mathrm{J}=7.5$ $\mathrm{Hz}, 1 \mathrm{H}, \mathrm{C}(7)-\mathbf{H}), 4.04\left(\mathrm{dd}, \mathrm{J}_{1}=8.5 \mathrm{~Hz}, \mathrm{~J}_{2}=7.5 \mathrm{~Hz}, 1 \mathrm{H}, \mathrm{C}(8)-\mathbf{H}\right), 3.87$ (t, $\mathrm{J}_{1}=8.5 \mathrm{~Hz}, \mathrm{~J}_{2}=7.5 \mathrm{~Hz}$, 1H, C(8)-H), 2.71 (s, 1H, C(1)-H), 1.51 (s, 3H, C(CH $\left.)_{2}\right), 1.36$ (s, 3H, C(CH $\left.)_{2}\right), 0.93$ (s, 9H, $\left.\mathrm{SiC}\left(\mathrm{CH}_{3}\right)_{3}\left(\mathrm{CH}_{3}\right)_{2}\right), 0.05\left(\mathrm{~s}, 6 \mathrm{H}, \mathrm{SiC}\left(\mathrm{CH}_{3}\right)_{3}\left(\mathrm{CH}_{3}\right)_{2}\right) .{ }^{13} \mathrm{C} \mathrm{NMR}\left(100 \mathrm{MHz}, \mathrm{C}_{6} \mathrm{D}_{6}\right), \delta 135.2,119.7$, 110.4, 105.6, 101.1, 84.1, 81.1, 74.9, 68.2, 26.6, 26.1, 16.8, -4.8. IR ( NaCl, thin film), $\mathrm{cm}^{-1} 3288$, 2987, 2953, 2930, 2884, 2858, 2166, 2122, 2093, 1471, 1372, 1251, 1066, 840. HRMS-ESI $(\mathrm{m} / \mathrm{z})$ $[\mathrm{M}+\mathrm{H}]^{+}$calcd for $\mathrm{C}_{17} \mathrm{H}_{27} \mathrm{O}_{2} \mathrm{Si}, 291.1780$; found, 291.1789 . 

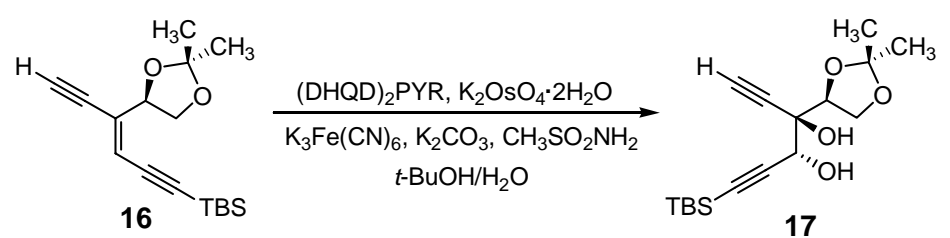

\section{1,5-Hexadiyne-3,4-diol 17}

A $100-\mathrm{mL}$, round-bottom flask was charged with $\mathrm{K}_{2} \mathrm{OsO}_{4} \cdot 2 \mathrm{H}_{2} \mathrm{O}(44.2 \mathrm{mg}, 0.120 \mathrm{mmol}$, 0.04 equiv), (DHQD) 2 PYR (529 mg, $0.600 \mathrm{mmol}, 0.20$ equiv), $\mathrm{K}_{3} \mathrm{Fe}(\mathrm{CN})_{6}(4.94 \mathrm{~g}, 15.0 \mathrm{mmol}, 5.0$ equiv), $\mathrm{K}_{2} \mathrm{CO}_{3}$ (2.07 g, $15.0 \mathrm{mmol}$. 5.0 equiv), $t$-BuOH $(5 \mathrm{~mL})$, and $\mathrm{H}_{2} \mathrm{O}(10 \mathrm{~mL})$. The mixture was stirred at $0{ }^{\circ} \mathrm{C}$ for $15 \mathrm{~min}$, and methanesulfonamide (1.14 g, $12.0 \mathrm{mmol}, 4.0$ equiv) was added. After $5 \mathrm{~min}$, a solution of the enediyne 16 (874 $\mathrm{mg}, 3.00 \mathrm{mmol}, 1.0$ equiv) in $t$-BuOH (5 mL) was added, and the reaction mixture was stirred at $0{ }^{\circ} \mathrm{C}$ for $16 \mathrm{~h}$. Saturated aqueous sodium bisulfite solution $(10 \mathrm{~mL})$ was added, and the resulting biphasic mixture was stirred at $23{ }^{\circ} \mathrm{C}$ for $30 \mathrm{~min}$. The mixture was diluted with EtOAc $(40 \mathrm{~mL})$ and the layers were separated. The aqueous layer was extracted with EtOAc $(3 \times 40 \mathrm{~mL})$. The organic extracts were combined and the combined solution was washed with brine $(30 \mathrm{~mL})$. The washed solution was dried over anhydrous sodium sulfate. The dried solution was filtered and the filtrate was concentrated. The residue was purified by flash-column chromatography $(25 \%$ EtOAc-hexanes $)$ to give the 1,5-hexadiyne-3,4-diol 17 (white foam, $518 \mathrm{mg}, 53 \%$ ) and, separately, recovered starting material (16, yellow oil, $60.2 \mathrm{mg}, 7 \%)$.

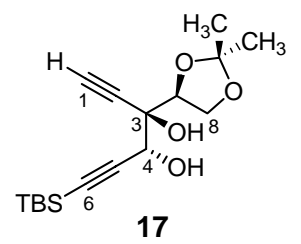

$\mathrm{R}_{f}=0.25$ (25\% ethyl acetate-hexanes). ${ }^{1} \mathrm{H}$ NMR (500 MHz, $\left.\mathrm{CDCl}_{3}\right), \delta 4.62$ (d, 1H, C(4)-H), 4.33 $(\mathrm{t}, 1 \mathrm{H}, \mathrm{J}=6.5 \mathrm{~Hz}, \mathrm{C}(7)-\mathbf{H}), 4.23(\mathrm{dd}, 1 \mathrm{H}, \mathrm{J}=6.0 \mathrm{~Hz}, \mathrm{C}(8)-\mathbf{H}), 4.16(\mathrm{dd}, 1 \mathrm{H}, \mathrm{J}=6.0 \mathrm{~Hz}, \mathrm{C}(8)-\mathbf{H})$, $2.81(\mathrm{~d}, 1 \mathrm{H}, \mathrm{J}=5.0 \mathrm{~Hz}, \mathrm{C}(4)-\mathrm{OH}), 2.79$ (s, 1H, C(3)-OH), 2.56 (s, 1H, C(1)-H), 1.49 (s, 3H, $\left.\mathrm{C}\left(\mathrm{CH}_{3}\right)_{2}\right), 1.37$ (s, 3H, C(CH$\left.)_{2}\right), 0.95$ (s, 9H, $\left.\mathrm{SiC}\left(\mathrm{CH}_{3}\right)_{3}\left(\mathrm{CH}_{3}\right)_{2}\right), 0.14$ (s, 6H, $\left.\mathrm{SiC}\left(\mathrm{CH}_{3}\right)_{3}\left(\mathrm{CH}_{3}\right)_{2}\right)$. ${ }^{13} \mathrm{C}$ NMR (100 MHz, $\left.\mathrm{CDCl}_{3}\right), \delta 110.4,101.3,91.6,81.2,78.8,75.3,73.6,68.6,66.2,26.2,26.0$, 25.2, 16.5, -4.8. IR ( $\mathrm{NaCl}$, thin film), $\mathrm{cm}^{-1} 3425,3307,2980,2949,2930,2886,2857,1460$, 1372, 1251. HRMS-ESI $(\mathrm{m} / \mathrm{z})[\mathrm{M}+\mathrm{H}]^{+}$calcd for $\mathrm{C}_{17} \mathrm{H}_{29} \mathrm{O}_{4} \mathrm{Si}, 325.1835$; found, 325.1842 .
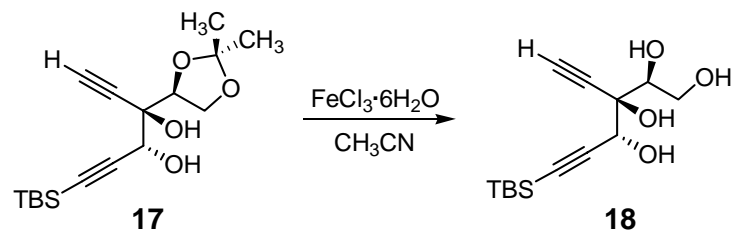

\section{Tetraol 18}

Iron (III) chloride hexahydrate (3.52 g, $13.0 \mathrm{mmol}, 3.0$ equiv) was added to a stirring solution of the 1,5-hexadiyne-3,4-diol 17 (1.41 g, $4.35 \mathrm{mmol}, 1$ equiv) in acetonitrile (40 mL) at 23 ${ }^{\circ} \mathrm{C}$. After stirring at $23{ }^{\circ} \mathrm{C}$ for $40 \mathrm{~min}$, the reaction mixture was concentrated to a volume of ca. 15 $\mathrm{mL}$. The concentrate was diluted with ethyl acetate $(100 \mathrm{~mL})$. The resulting solution was 
washed with water $(80 \mathrm{~mL})$ and the layers were separated. The aqueous layer was extracted with ethyl acetate $(2 \times 80 \mathrm{~mL})$. The organic extracts were combined and the combined solution was washed with brine $(2 \times 50 \mathrm{~mL})$. The washed organic solution was dried over anhydrous sodium sulfate. The dried solution was filtered and the filtrate was concentrated. The residue was purified by flash-column chromatography (60\% ethyl acetate-hexanes) to provide the tetraol $\mathbf{1 8}$ (colorless oil, $1.24 \mathrm{~g}, 100 \%$ ).

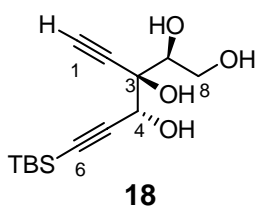

$\mathrm{R}_{f}=0.20$ (60\% ethyl acetate-hexanes). ${ }^{1} \mathrm{H}$ NMR $\left(500 \mathrm{MHz}, \mathrm{CDCl}_{3}\right), \delta 4.65$ (s, br, $\left.1 \mathrm{H}, \mathrm{C}(4)-\mathrm{H}\right)$, 3.99-3.92 (m, 3H, C(7)-H + C(8)-H), 3.60 (s, br, 1H, -OH), 3.36 (s, br, 1H, -OH), 3.18 (s, br, 1H, -OH), 2.76 (s, br, 1H, -OH), 2.62 (s, 1H, C(1)-H), 0.95 (s, 9H, $\left.\mathrm{SiC}\left(\mathrm{CH}_{3}\right)_{3}\left(\mathrm{CH}_{3}\right)_{2}\right), 0.14$ (s, 6H, $\left.\mathrm{SiC}\left(\mathrm{CH}_{3}\right)_{3}\left(\mathrm{CH}_{3}\right)_{2}\right) .{ }^{13} \mathrm{C} \mathrm{NMR}\left(100 \mathrm{MHz}, \mathrm{CDCl}_{3}\right), \delta$ 101.9, 91.8, 81.3, 76.2, 74.6, 74.3, 67.2, 62.5, 26.0, 16.5, -4.8. IR ( $\mathrm{NaCl}$, thin film), $\mathrm{cm}^{-1} 3393,2928,2857,2177,2113,1467,1250,1071$. HRMS-ESI $(\mathrm{m} / z)\left[\mathrm{M}+\mathrm{NH}_{4}\right]^{+}$calcd for $\mathrm{C}_{14} \mathrm{H}_{28} \mathrm{NO}_{4} \mathrm{Si}, 302.1788$; found, 302.1775 .

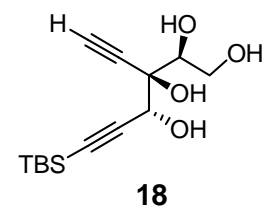

18

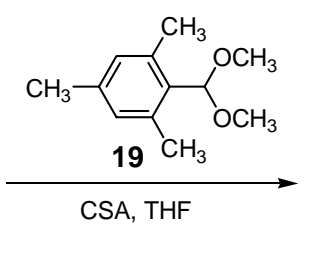

(major diastereomer)

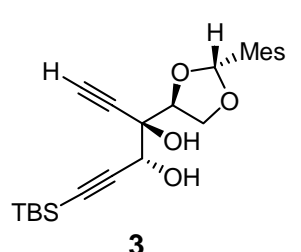

\section{1,5-Hexadiyne-3,4-diol 3}

Camphorsulfonic acid monohydrate $(71.8 \mathrm{mg}, 0.309 \mathrm{mmmol}, 0.04$ equiv) was added to a solution of the tetraol 18 (2.20 g, $7.73 \mathrm{mmol}, 1$ equiv) and mesitaldehyde dimethyl acetal (19) ${ }^{7}$ (3.01 g, $15.5 \mathrm{mmol}, 2.0$ equiv) in tetrahydrofuran $(300 \mathrm{~mL})$ at $23{ }^{\circ} \mathrm{C}$. After stirring at $23{ }^{\circ} \mathrm{C}$ for 5 $\mathrm{h}$, the reaction mixture was partitioned between ethyl acetate $(100 \mathrm{~mL})$ and saturated aqueous sodium bicarbonate solution $(150 \mathrm{~mL})$. The layers were separated and the aqueous layer was extracted with ethyl acetate $(2 \times 100 \mathrm{~mL})$. The organic layers were combined and the combined solution was washed with brine $(60 \mathrm{~mL})$. The washed solution was dried over anhydrous sodium sulfate. The dried solution was filtered and the filtrate was concentrated. The residue was purified by flash-column chromatography (25\% ethyl acetate-hexanes) to provide the 1,5-hexadiyne-3,4-diol 3 (colorless oil, $1.76 \mathrm{~g}, 55 \%$ ) and, separately, the corresponding minor diastereomer (colorless oil, $0.45 \mathrm{~g}, 14 \%$ ).

\footnotetext{
${ }^{7}$ Mesitaldehyde dimethyl acetal (19) was synthesized as follows: camphursulfonic acid (10 mg) was added to a solution of mesitaldehyde ( $3.00 \mathrm{~g}, 20.2 \mathrm{mmol}, 1$ equiv) and trimethyl orthoformate ( $3.22 \mathrm{~g}, 30.4 \mathrm{mmol}, 1.5$ equiv) in methanol $(30 \mathrm{~mL})$ at $23{ }^{\circ} \mathrm{C}$. After stirring at $23{ }^{\circ} \mathrm{C}$ for $12 \mathrm{~h}$, the reaction mixture was partitioned between ether $(30$ $\mathrm{mL}$ ) and saturated aqueous sodium bicarbonate solution $(30 \mathrm{~mL})$. The aqueous layer was separated and further extracted with ether $(30 \mathrm{~mL})$. The organic extracts were combined and the combined solution was dried over anhydrous sodium sulfate. The dried solution was filtered and the filtrate was concentrated to provide mesitaldehyde dimethyl acetal (19) (colorless oil, $3.90 \mathrm{~g}, 99 \%$ ).
} 


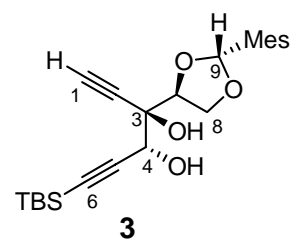

$\mathrm{R}_{f}=0.25$ (25\% ethyl acetate-hexanes). ${ }^{1} \mathrm{H}$ NMR $\left(500 \mathrm{MHz}, \mathrm{CDCl}_{3}\right), \delta 6.82(\mathrm{~s}, 2 \mathrm{H}, \mathrm{Ar}-\mathrm{H}), 6.41(\mathrm{~s}$, 1H, C(9)-H), 4.72 (d, J = 5.0 Hz, 1H, C(4)-H), 4.53-4.34 (m, 3H, C(7)-H + C(8)-H), 2.82 (s, 1H, $\mathrm{C}(3)-\mathrm{OH}), 2.81$ (d, J = 4.5 Hz, 1H, C(4)-OH), 2.66 (s, 1H, C(1)-H), 2.37 (s, 6H, Ar-CH ) $^{2}, 2.25$ (s, $\left.3 \mathrm{H}, \mathrm{Ar}-\mathrm{CH}_{3}\right), 0.95$ (s, 9H, $\left.\mathrm{SiC}\left(\mathrm{CH}_{3}\right)_{3}\left(\mathrm{CH}_{3}\right)_{2}\right), 0.14$ (s, 3H, $\left.\mathrm{SiC}\left(\mathrm{CH}_{3}\right)_{3}\left(\mathrm{CH}_{3}\right)_{2}\right), 0.13$ (s, 3H, $\left.\mathrm{SiC}\left(\mathrm{CH}_{3}\right)_{3}\left(\mathrm{CH}_{3}\right)_{2}\right) .{ }^{13} \mathrm{C} \mathrm{NMR}\left(100 \mathrm{MHz}, \mathrm{CDCl}_{3}\right), \delta$ 139.1, 138.1, 130.0, 127.8, 103.3, 101.0, 92.1, 81.6, 77.6, 76.4, 74.4, 69.2, 68.3, 26.0, 20.9, 19.8, 16.5, -4.8 (2). IR ( NaCl, thin film), $\mathrm{cm}^{-1} 3416$, $3306,2928,2857,2177,2116,1614,1470,1251,1077 .[\alpha]^{23}-26.9^{\circ}\left(\mathrm{c} 0.90, \mathrm{CHCl}_{3}\right)$. HRMS-ESI $(m / z)[\mathrm{M}+\mathrm{H}]^{+}$calcd for $\mathrm{C}_{24} \mathrm{H}_{35} \mathrm{O}_{4} \mathrm{Si}, 415.2304$; found, 415.2313 .

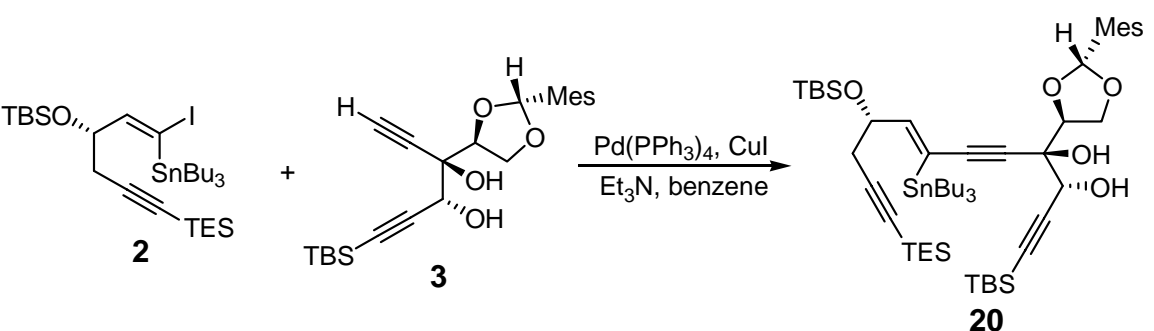

\section{Enetriyne Diol 20}

A mixture of the (1-iodovinyl) stannane $2(2.57 \mathrm{~g}, 3.47 \mathrm{mmol}, 1.8$ equiv), the 1,5-hexadiyne-3,4-diol 3 (800 mg, $1.93 \mathrm{mmol}, 1$ equiv), tetrakis(triphenylphosphine)palladium (669 mg, $0.579 \mathrm{mmol}, 0.3$ equiv), and copper(I) iodide ( $221 \mathrm{mg}, 1.16 \mathrm{mmol}, 0.6$ equiv) in benzene $(12 \mathrm{~mL})$ was subjected to iterative freeze-pump-thaw cycles $(4 \times)$. Triethylamine $(807 \mu \mathrm{L}, 5.79$ mmol, 3.0 equiv) was then added to the deoxygenated solution at $23{ }^{\circ} \mathrm{C}$. The mixture was stirred at $23{ }^{\circ} \mathrm{C}$ for $21 \mathrm{~h}$, then $N$-acetyl cysteine $(5 \mathrm{~g})$ and ether $(15 \mathrm{~mL})$ were added, followed by triethylamine $(3 \mathrm{~mL})$. After stirring at $23{ }^{\circ} \mathrm{C}$ for $1 \mathrm{~h}$, the mixture obtained was partitioned between ethyl acetate $(100 \mathrm{~mL})$ and a 1:1 mixture of saturated aqueous ammonium chloride solution and water $(100 \mathrm{~mL})$. The aqueous layer was separated and extracted with ethyl acetate $(2 \times 100 \mathrm{~mL})$. The organic extracts were combined and the combined solution was washed with brine $(60 \mathrm{~mL})$. The washed solution was dried over anhydrous sodium sulfate. The dried solution was filtered and the filtrate was concentrated. The residue was purified by flash-column chromatography ( $8 \%$ ethyl acetate-hexanes) to provide the enetriyne diol $\mathbf{2 0}$ (colorless oil, $1.03 \mathrm{~g}, 64 \%$ ).

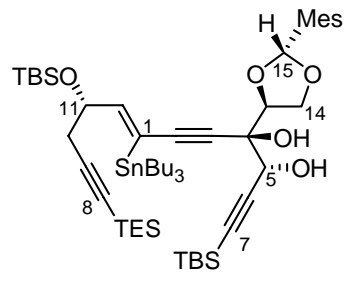

20

$\mathrm{R}_{f}=0.25$ (17\% ether-hexanes). $\quad{ }^{1} \mathrm{H}$ NMR $\left(600 \mathrm{MHz}, \mathrm{CDCl}_{3}\right), \delta 6.81$ (s, 2H, Ar-H), 6.72 (ddt, $\mathrm{J}_{1}=$ $\left.2.4 \mathrm{~Hz}, \mathrm{~J}_{2}=8.4 \mathrm{~Hz}, \mathrm{~J}_{3}=50.4 \mathrm{~Hz}, 1 \mathrm{H}, \mathrm{C}(12)-\mathbf{H}\right), 6.39(\mathrm{~s}, 1 \mathrm{H}, \mathrm{C}(15)-\mathbf{H}), 4.72(\mathrm{~d}, \mathrm{~J}=4.8 \mathrm{~Hz}, 1 \mathrm{H}$, 
$\mathrm{C}(5)-\mathbf{H}), 4.52(\mathrm{~m}, 2 \mathrm{H}, \mathrm{C}(13)-\mathbf{H}+\mathrm{C}(14)-\mathbf{H}), 4.34$ (t, J = 7.2 Hz, 1H, C(14)-H), 4.16 (dt, J $=3.0 \mathrm{~Hz}$, $\left.\mathrm{J}_{2}=8.4 \mathrm{~Hz}, 1 \mathrm{H}, \mathrm{C}(11)-\mathbf{H}\right), 2.70$ (s, 1H, C(4)-OH), 2.61 (d, J = 4.2 Hz, 1H, C(5)-OH), 2.47 (dd, J = $\left.8.4 \mathrm{~Hz}, \mathrm{~J}_{2}=16.8 \mathrm{~Hz}, 1 \mathrm{H}, \mathrm{C}(10)-\mathbf{H}\right), 2.37$ (s, 6H, Ar-CH $\left.{ }_{3}\right), 2.34\left(\mathrm{dd}, \mathrm{J}_{1}=3.0 \mathrm{~Hz}, \mathrm{~J}_{2}=16.8 \mathrm{~Hz}, 1 \mathrm{H}\right.$, $\mathrm{C}(10)-\mathbf{H}), \quad 2.25$ (s, 3H, Ar-CH $\mathrm{H}_{3}, \quad 1.55 \quad\left(\mathrm{~m}, \quad 6 \mathrm{H}, \quad \mathrm{Sn}\left(\mathrm{CH}_{2} \mathrm{CH}_{2} \mathrm{CH}_{2} \mathrm{CH}_{3}\right)_{3}, \quad 1.35 \quad(\mathrm{~m}, \quad 6 \mathrm{H}\right.$, $\left.\mathrm{Sn}\left(\mathrm{CH}_{2} \mathrm{CH}_{2} \mathrm{CH}_{2} \mathrm{CH}_{3}\right)_{3}\right), 1.11\left(\mathrm{~m}, 6 \mathrm{H}, \mathrm{Sn}\left(\mathrm{CH}_{2} \mathrm{CH}_{2} \mathrm{CH}_{2} \mathrm{CH}_{3}\right)_{3}, 0.99\right.$ (t, J = $\left.7.8 \mathrm{~Hz}, \mathrm{Si}\left(\mathrm{CH}_{2} \mathrm{CH}_{3}\right)_{3}\right)$, 0.95 (s, 9H, $\left.\mathrm{Si}\left(\mathrm{CH}_{3}\right)_{2} \mathrm{C}\left(\mathrm{CH}_{3}\right)_{3}\right), 0.91$ (s, 9H, $\left.\mathrm{Si}\left(\mathrm{CH}_{3}\right)_{2} \mathrm{C}\left(\mathrm{CH}_{3}\right)_{3}\right), 0.91$ (t, J = $7.2 \mathrm{~Hz}, 9 \mathrm{H}$, $\left.\mathrm{Sn}\left(\mathrm{CH}_{2} \mathrm{CH}_{2} \mathrm{CH}_{2} \mathrm{CH}_{3}\right)_{3}\right), 0.58$ (q, J = $\left.7.8 \mathrm{~Hz}, 6 \mathrm{H}, \mathrm{Si}\left(\mathrm{CH}_{2} \mathrm{CH}_{3}\right)_{3}\right), 0.14(2)\left(\mathrm{s}, 3 \mathrm{H}, \mathrm{Si}\left(\mathrm{CH}_{3}\right)_{2} \mathrm{C}\left(\mathrm{CH}_{3}\right)_{3}\right)$, 0.13 (s, 3H, $\left.\mathrm{Si}\left(\mathrm{CH}_{3}\right)_{2} \mathrm{C}\left(\mathrm{CH}_{3}\right)_{3}\right), 0.08$ (s, 3H, $\left.\mathrm{Si}\left(\mathrm{CH}_{3}\right)_{2} \mathrm{C}\left(\mathrm{CH}_{3}\right)_{3}\right) .{ }^{13} \mathrm{C} \mathrm{NMR}\left(100 \mathrm{MHz}, \mathrm{CDCl}_{3}\right), \delta$ 156.3, 138.8, 138.0, 130.0, 128.1, 123.3, 104.4, 103.1, 101.7, 91.9, 91.5, 89.4, 83.6, 77.8, 75.4, $74.4,69.2,68.5,30.8,29.0,27.3,26.0,25.8,20.9,20.0,18.1,16.5,13.6,11.2\left(\mathrm{dt}, \mathrm{J}_{1}=7.6 \mathrm{~Hz}, \mathrm{~J}_{2}=\right.$ $166 \mathrm{~Hz}), 7.4,4.4,-4.1,-4.3,-4.8(2)$. IR (NaCl, thin film) $\mathrm{cm}^{-1} 3445,2952,2174,1615,1464$, 1456. $[\alpha]^{23}-50.3^{\circ}\left(\mathrm{c} 0.33, \mathrm{CHCl}_{3}\right)$. HRMS-ESI $(\mathrm{m} / \mathrm{z})[\mathrm{M}+\mathrm{H}]^{+}$calcd for $\mathrm{C}_{54} \mathrm{H}_{95} \mathrm{O}_{5} \mathrm{Si}_{3} \mathrm{Sn}$, 1027.5509; found, 1027.5500 .

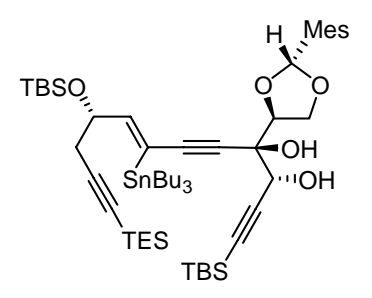

20

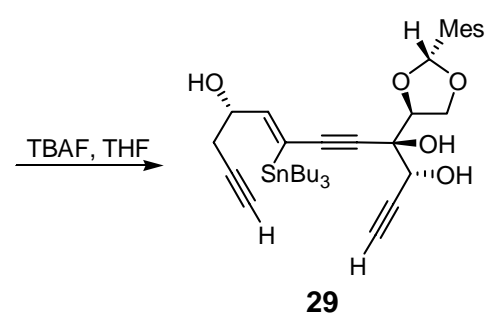

29

\section{Enetriyne Triol 29}

A solution of tetrabutylammonium fluoride in tetrahydrofuran $(1.0 \mathrm{M}, 2.62 \mathrm{~mL}, 2.62 \mathrm{mmol}$, 3.05 equiv) was added to a solution of the enetriyne diol 20 ( $0.88 \mathrm{~g}, 0.857 \mathrm{mmol}, 1$ equiv) in tetrahydrofuran $(25 \mathrm{~mL})$ at $0{ }^{\circ} \mathrm{C}$. The reaction mixture was stirred at $0{ }^{\circ} \mathrm{C}$ for $2.5 \mathrm{~h}$. Saturated aqueous ammonium chloride solution $(40 \mathrm{~mL})$ and water $(10 \mathrm{~mL})$ were then added and tetrahydrofuran was removed in vacuo. The residual biphasic mixture was extracted with ethyl acetate $(2 \times 50 \mathrm{~mL})$. The organic extracts were combined and the combined solution was washed with brine $(20 \mathrm{~mL})$. The washed organic solution was dried over anhydrous sodium sulfate. The dried solution was filtered and the filtrate was concentrated. The residue was purified by flash-column chromatography (50\% ether-hexanes) to provide the enetriyne triol 29 (yellow oil, $534 \mathrm{mg}, 91 \%)$.

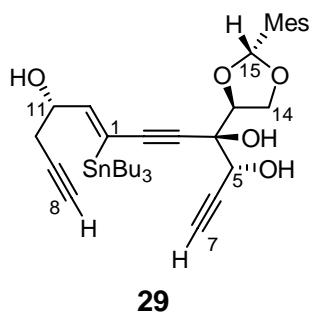

$\mathrm{R}_{f}=0.32$ (50\% ether-hexanes). ${ }^{1} \mathrm{H}$ NMR $\left(500 \mathrm{MHz}, \mathrm{CDCl}_{3}\right), \delta 6.81(\mathrm{~s}, 2 \mathrm{H}, \mathrm{Ar}-\mathbf{H}), 6.78\left(\mathrm{ddt}, \mathrm{J}_{1}=\right.$ $\left.2.5 \mathrm{~Hz}, \mathrm{~J}_{2}=7.0 \mathrm{~Hz}, \mathrm{~J}_{3}=47.5 \mathrm{~Hz}, 1 \mathrm{H}, \mathrm{C}(12)-\mathbf{H}\right), 6.40$ (s, 1H, C(15)-H), $4.71\left(\mathrm{dd}, \mathrm{J}_{1}=2.0 \mathrm{~Hz}, \mathrm{~J}_{2}=\right.$ $4.5 \mathrm{~Hz}, 1 \mathrm{H}, \mathrm{C}(5)-\mathbf{H}), 4.50(\mathrm{~m}, 2 \mathrm{H}, \mathrm{C}(13)-\mathbf{H}+\mathrm{C}(14)-\mathbf{H}), 4.34(\mathrm{~m}, 1 \mathrm{H}, \mathrm{C}(14)-\mathbf{H}), 4.22(\mathrm{~m}, 1 \mathrm{H}$, $\mathrm{C}(11)-\mathbf{H}), 2.82$ (d, J = 4.5 Hz, 1H, C(5)-OH), 2.72 (s, 1H, C(4)-OH), 2.58 (d, J = 2.0 Hz, 1H, $\mathrm{C}(7)-\mathbf{H}), 2.51$ (ddd, $\left.\mathrm{J}_{1}=2.5 \mathrm{~Hz}, \mathrm{~J}_{2}=5.0 \mathrm{~Hz}, \mathrm{~J}_{3}=16.5 \mathrm{~Hz}, 1 \mathrm{H}, \mathrm{C}(10)-\mathbf{H}\right), 2.42$ (ddd, $\mathrm{J}_{1}=2.5 \mathrm{~Hz}, \mathrm{~J}_{2}$ 
$\left.=7.0 \mathrm{~Hz}, \mathrm{~J}_{3}=16.5 \mathrm{~Hz}, 1 \mathrm{H}, \mathrm{C}(10)-\mathbf{H}\right), 2.37\left(\mathrm{~s}, 6 \mathrm{H}, \mathrm{Ar}-\mathrm{CH}_{3}\right), 2.25\left(\mathrm{~s}, 3 \mathrm{H}, \mathrm{Ar}-\mathrm{CH} \mathbf{H}_{3}\right), 2.10(\mathrm{t}, \mathrm{J}=2.5$ $\mathrm{Hz}, 1 \mathrm{H}, \mathrm{C}(8)-\mathrm{H}), 2.06$ (d, J = 4.5 Hz, 1H, C(11)-OH), $1.53\left(\mathrm{~m}, 6 \mathrm{H}, \mathrm{Sn}\left(\mathrm{CH}_{2} \mathrm{CH}_{2} \mathrm{CH}_{2} \mathrm{CH}_{3}\right)_{3}, 1.33(\mathrm{~m}\right.$, $\left.6 \mathrm{H}, \mathrm{Sn}\left(\mathrm{CH}_{2} \mathrm{CH}_{2} \mathrm{CH}_{2} \mathrm{CH}_{3}\right)_{3}\right), 1.08\left(\mathrm{~m}, 6 \mathrm{H}, \mathrm{Sn}\left(\mathrm{CH}_{2} \mathrm{CH}_{2} \mathrm{CH}_{2} \mathrm{CH}_{3}\right)_{3}\right), 0.90$ (t, J = 7.2 Hz, 9H, $\left.\mathrm{Sn}\left(\mathrm{CH}_{2} \mathrm{CH}_{2} \mathrm{CH}_{2} \mathrm{CH}_{3}\right)_{3}\right) .{ }^{13} \mathrm{C} \mathrm{NMR}\left(100 \mathrm{MHz}, \mathrm{CDCl}_{3}\right), \delta$ 152.2, 138.9, 138.0, 130.0, 127.9, 127.3, 103.1, 92.3, 90.0, 79.9, 79.6, 77.9, 75.8, 75.2, 71.6, 71.5, 68.9, 68.4, $29.0(\mathrm{t}, \mathrm{J}=7.8 \mathrm{~Hz}), 27.6,27.3$ $(\mathrm{t}, \mathrm{J}=24.4 \mathrm{~Hz}), 20.9,20.0,13.7,11.9\left(\mathrm{dt}, \mathrm{J}_{1}=6.1 \mathrm{~Hz}, \mathrm{~J}_{2}=136 \mathrm{~Hz}\right) . \quad \mathrm{IR}\left(\mathrm{NaCl}\right.$, thin film), $\mathrm{cm}^{-1}$ 3445, 3307, 2955, 2922, 2868, 2858, 2357, 2335, 2190, 2116, 1220, 1155, 1075, 1044, 949, 924, 850. HRMS-ESI $(\mathrm{m} / \mathrm{z})[\mathrm{M}+\mathrm{H}]^{+}$calcd for $\mathrm{C}_{36} \mathrm{H}_{53} \mathrm{O}_{5} \mathrm{Sn}, 685.2915$; found, 685.2930.

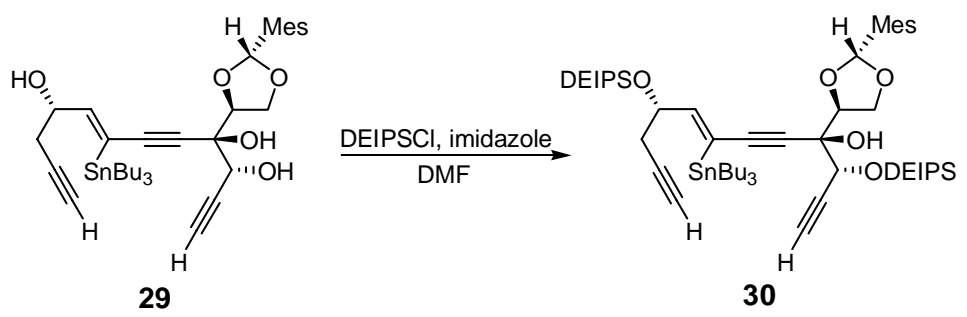

\section{Bis(diethylisopropylsilyl) Ether 30}

Chlorodiethylisopropylsilane ( $577 \mu \mathrm{L}, 3.13 \mathrm{mmol}, 4.0$ equiv) was added via syringe over 2 min to a stirring solution of the enetriyne triol $29(534 \mathrm{mg}, 0.781 \mathrm{mmol}, 1$ equiv) and imidazole (375 mg, $6.25 \mathrm{mmol}, 8.0$ equiv) in $N, N$-dimethylformamide $(12 \mathrm{~mL})$ at $0{ }^{\circ} \mathrm{C}$. After stirring at 0 ${ }^{\circ} \mathrm{C}$ for $1.5 \mathrm{~h}$, the reaction mixture was partitioned between ethyl acetate $(40 \mathrm{~mL})$ and water $(40 \mathrm{~mL})$. The aqueous layer was separated and extracted with ethyl acetate $(40 \mathrm{~mL})$. The organic extracts were combined and the combined solution was washed with brine $(20 \mathrm{~mL})$. The washed organic solution was dried over anhydrous sodium sulfate. The dried solution was filtered and the filtrate was concentrated. The residue was purified by flash-column chromatography (4\% ethyl acetate-hexanes) to provide the bis(diethylisopropylsilyl) ether $\mathbf{3 0}$ (colorless oil, $653 \mathrm{mg}, 89 \%$ ).

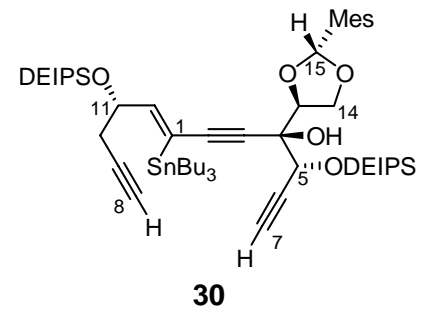

$\mathrm{R}_{f}=0.60$ (13\% ether-hexanes). ${ }^{1} \mathrm{H}$ NMR $\left(500 \mathrm{MHz}, \mathrm{CDCl}_{3}\right), \delta 6.81(\mathrm{~s}, 2 \mathrm{H}, \mathrm{Ar}-\mathrm{H}), 6.78$ (ddt, $\mathrm{J}_{1}=$ $\left.2.5 \mathrm{~Hz}, \mathrm{~J}_{2}=8.5 \mathrm{~Hz}, \mathrm{~J}_{3}=51.3 \mathrm{~Hz}, 1 \mathrm{H}, \mathrm{C}(12)-\mathbf{H}\right), 6.39(\mathrm{~s}, 1 \mathrm{H}, \mathrm{C}(15)-\mathrm{H}), 4.77(\mathrm{~d}, \mathrm{~J}=2.5 \mathrm{~Hz}, 1 \mathrm{H}$, $\mathrm{C}(5)-\mathbf{H}), 4.61(\mathrm{t}, \mathrm{J}=7.0 \mathrm{~Hz}, 1 \mathrm{H}, \mathrm{C}(13)-\mathbf{H}), 4.51\left(\mathrm{dd}, \mathrm{J}_{1}=7.0 \mathrm{~Hz}, \mathrm{~J}_{2}=8.5 \mathrm{~Hz}, 1 \mathrm{H}, \mathrm{C}(14)-\mathbf{H}\right), 4.34(\mathrm{t}$, $\mathrm{J}=8.5 \mathrm{~Hz}, 1 \mathrm{H}, \mathrm{C}(14)-\mathbf{H}), 4.17(\mathrm{~m}, 1 \mathrm{H}, \mathrm{C}(11)-\mathbf{H}), 2.99(\mathrm{~s}, 1 \mathrm{H}, \mathrm{C}(4)-\mathrm{OH}), 2.51(\mathrm{~d}, \mathrm{~J}=2.0 \mathrm{~Hz}, 1 \mathrm{H}$, $\mathrm{C}(7)-\mathbf{H}), 2.43$ (ddd, $\left.\mathrm{J}_{1}=3.0 \mathrm{~Hz}, \mathrm{~J}_{2}=6.0 \mathrm{~Hz}, \mathrm{~J}_{3}=16.5 \mathrm{~Hz}, 1 \mathrm{H}, \mathrm{C}(10)-\mathbf{H}\right), 2.38\left(\mathrm{~s}, 6 \mathrm{H}, \mathrm{Ar}-\mathrm{CH}_{3}\right), 2.31$ $\left(\mathrm{ddd}, \mathrm{J}_{1}=3.0 \mathrm{~Hz}, \mathrm{~J}_{2}=6.0 \mathrm{~Hz}, \mathrm{~J}_{3}=16.5 \mathrm{~Hz}, 1 \mathrm{H}, \mathrm{C}(10)-\mathrm{H}\right), 2.25\left(\mathrm{~s}, 3 \mathrm{H}, \mathrm{Ar}-\mathrm{CH}_{3}\right), 1.95(\mathrm{t}, \mathrm{J}=2.5 \mathrm{~Hz}$, $1 \mathrm{H}, \mathrm{C}(8)-\mathrm{H}), 1.55\left(\mathrm{~m}, 6 \mathrm{H}, \mathrm{Sn}\left(\mathrm{CH}_{2} \mathrm{CH}_{2} \mathrm{CH}_{2} \mathrm{CH}_{3}\right)_{3}, 1.35\right.$ (m, 6H, Sn( $\left.\left(\mathrm{CH}_{2} \mathrm{CH}_{2} \mathrm{CH}_{2} \mathrm{CH}_{3}\right)_{3}\right), 1.11$ (m, 6H, $\left.\mathrm{Sn}\left(\mathrm{CH}_{2} \mathrm{CH}_{2} \mathrm{CH}_{2} \mathrm{CH}_{3}\right)_{3}\right), 1.04-0.96\left(\mathrm{~m}, 26 \mathrm{H}, \mathrm{Si}\left(\mathrm{CH}_{2} \mathrm{CH}_{3}\right)_{2} \mathrm{CH}\left(\mathrm{CH}_{3}\right)_{2}\right), 0.91(\mathrm{t}, \mathrm{J}=7.5 \mathrm{~Hz}, 9 \mathrm{H}$, $\left.\mathrm{Sn}\left(\mathrm{CH}_{2} \mathrm{CH}_{2} \mathrm{CH}_{2} \mathrm{CH}_{3}\right)_{3}\right), \quad 0.79-0.70 \quad\left(\mathrm{~m}, \quad 4 \mathrm{H}, \quad \mathrm{Si}\left(\mathrm{CH}_{2} \mathrm{CH}_{3}\right)_{2} \mathrm{CH}\left(\mathrm{CH}_{3}\right)_{2}\right), \quad 0.69-0.59 \quad(\mathrm{~m}, \quad 4 \mathrm{H}$, $\left.\mathrm{Si}\left(\mathrm{CH}_{2} \mathrm{CH}_{3}\right)_{2} \mathrm{CH}\left(\mathrm{CH}_{3}\right)_{2}\right) .{ }^{13} \mathrm{C}$ NMR $\left(100 \mathrm{MHz}, \mathrm{CDCl}_{3}\right), \delta 154.4,138.7,138.1,130.0,128.5$, 124.2, 102.9, 91.5, 90.8, 81.0, 80.6, 76.8, 75.8, 75.2, 73.3, 70.3, 68.8, 68.5, 29.1, 27.4, 20.9, 20.0, 
17.3, 13.6, 13.1, 12.9, $11.3\left(\mathrm{dt}, \mathrm{J}_{1}=7.6 \mathrm{~Hz}, \mathrm{~J}_{2}=166 \mathrm{~Hz}\right), 7.1,7.0,4.0,3.9,3.6,3.5 . \quad \mathrm{IR}(\mathrm{NaCl}$, thin film), $\mathrm{cm}^{-1} 3560,3312,2957,2731,2189,2122,1615,1463,1080,1015,950,835,729,640$. $[\alpha]^{23}{ }_{D}-48.7^{\circ}$ (c $0.53, \mathrm{CHCl}_{3}$ ). HRMS-ESI $(\mathrm{m} / \mathrm{z})[\mathrm{M}+\mathrm{H}]^{+}$calcd for $\mathrm{C}_{50} \mathrm{H}_{85} \mathrm{O}_{5} \mathrm{Si} 2 \mathrm{Sn}, 941.4957$; found, 941.4928 .
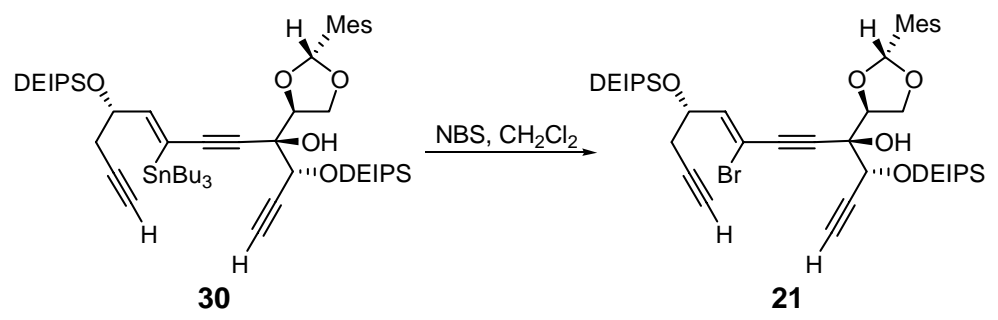

\section{Bromoenetriyne 21}

$\mathrm{N}$-Bromosuccinimide (161 $\mathrm{mg}, 0.903 \mathrm{mmol}, 1.3$ equiv) was added in one portion to a solution of the bis(diethylisopropylsilyl) ether $30(653 \mathrm{mg}, 0.695 \mathrm{mmol}, 1$ equiv) in dichloromethane $(8 \mathrm{~mL})$ at $23{ }^{\circ} \mathrm{C}$. After stirring at $23{ }^{\circ} \mathrm{C}$ for $12 \mathrm{~h}$, the reaction mixture was concentrated. The residue was purified by flash chromatography (7\% ethyl acetate-hexanes) to provide the bromoenetriyne 21 (colorless oil, $377 \mathrm{mg}, 89 \%$ ).

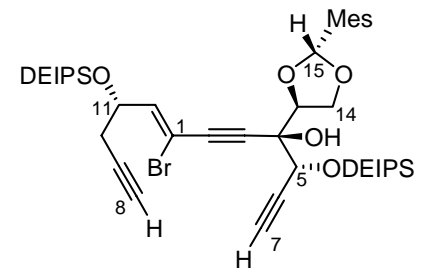

21

$\mathrm{R}_{f}=0.20$ (14\% ether-hexanes). ${ }^{1} \mathrm{H}$ NMR (500 MHz, $\left.\mathrm{C}_{6} \mathrm{D}_{6}\right), \delta 6.82(\mathrm{~s}, 1 \mathrm{H}, \mathrm{C}(15)-\mathrm{H}), 6.71(\mathrm{~s}, 2 \mathrm{H}$, $\operatorname{Ar}-\mathbf{H}), 6.52(\mathrm{~d}, \mathrm{~J}=6.5 \mathrm{~Hz}, 1 \mathrm{H}, \mathrm{C}(12)-\mathrm{H}), 5.01(\mathrm{~d}, \mathrm{~J}=2.0 \mathrm{~Hz}, 1 \mathrm{H}, \mathrm{C}(5)-\mathrm{H}), 4.89$ (t, J = $6.0 \mathrm{~Hz}, 1 \mathrm{H}$, C(13)-H), 4.81 (m, 1H, C(11)-H), 4.59 (t, J = 7.0 Hz, 1H, C(14)-H), 4.52 (dd, J $\mathrm{J}_{1}=5.5 \mathrm{~Hz}, \mathrm{~J}_{2}=7.5$ $\mathrm{Hz}, 1 \mathrm{H}, \mathrm{C}(14)-\mathbf{H}), 3.15$ (s, 1H, C(4)-OH), 2.54 (s, 6H, Ar-CH ) $^{2}, 2.36$ (ddd, $\mathrm{J}_{1}=2.0 \mathrm{~Hz}, \mathrm{~J}_{2}=5.0 \mathrm{~Hz}$, $\left.\mathrm{J}_{3}=13.5 \mathrm{~Hz}, 1 \mathrm{H}, \mathrm{C}(10)-\mathbf{H}\right), 2.31\left(\mathrm{ddd}, \mathrm{J}_{1}=2.5 \mathrm{~Hz}, \mathrm{~J}_{2}=5.0 \mathrm{~Hz}, \mathrm{~J}_{3}=14.0 \mathrm{~Hz}, 1 \mathrm{H}, \mathrm{C}(10)-\mathbf{H}\right), 2.07(\mathrm{~s}$, $\left.3 \mathrm{H}, \mathrm{Ar}-\mathrm{CH}_{3}\right), 2.00$ (d, J = 2.0 Hz, 1H, C(7)-H), 1.74 (t, J = 2.5 Hz, 1H, C(8)-H), 1.09-0.90 (m, 26H, $\left.\mathrm{Si}\left(\mathrm{CH}_{2} \mathrm{CH}_{3}\right)_{2} \mathrm{CH}\left(\mathrm{CH}_{3}\right)_{2}\right), 0.75-0.69\left(\mathrm{~m}, 8 \mathrm{H}, \mathrm{Si}\left(\mathrm{CH}_{2} \mathrm{CH}_{3}\right)_{2} \mathrm{CH}\left(\mathrm{CH}_{3}\right)_{2}\right) .{ }^{13} \mathrm{C}$ NMR (100 MHz, $\left.\mathrm{C}_{6} \mathrm{D}_{6}\right), \delta$ 142.5, 138.6, 138.4, 130.4, 129.3, 104.0, 101.5, 91.6, 84.6, 80.5, 79.7, 77.3, 76.5, 76.1, 71.2, 71.0, 69.0(2), 27.4, 20.9, 20.3, 17.5, 17.4, 13.2(2), 7.3, 7.2(2), 4.0(2), 3.9, 3.7. IR ( $\mathrm{NaCl}$, thin film), $\mathrm{cm}^{-1} 3543,3311,2955,2877,2359,2343,2121,1615,1463,1082 . \quad[\alpha]^{23}{ }_{\mathrm{D}}-101^{\circ}(\mathrm{c} 2.0$, $\left.\mathrm{CHCl}_{3}\right)$. HRMS-ESI $(\mathrm{m} / z)[\mathrm{M}+\mathrm{H}]^{+}$calcd for $\mathrm{C}_{38} \mathrm{H}_{58} \mathrm{BrO}_{5} \mathrm{Si}_{2}, 729.3006$; found, 729.3002 .
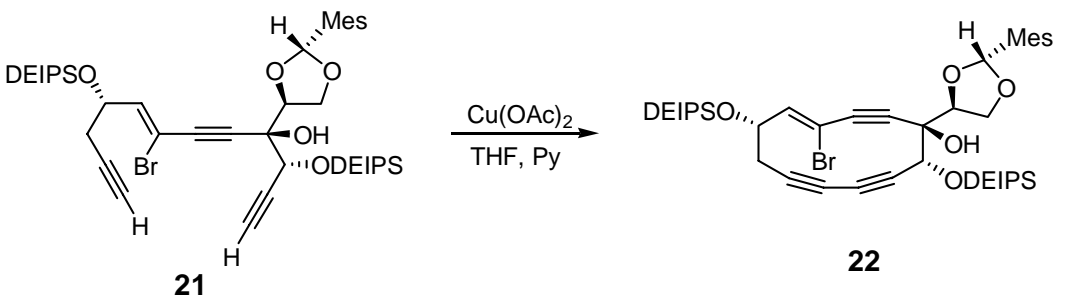

\section{Cyclic Bromoenetriyne 22}


A $100-\mathrm{mL}$, round-bottom flask was charged under an argon atmosphere with $\mathrm{Cu}(\mathrm{OAc})_{2}$ (918 mg, $5.06 \mathrm{mmol}, 60$ equiv), tetrahydrofuran $(15 \mathrm{~mL})$, and pyridine $(15 \mathrm{~mL})$, in that order. The mixture was stirred at $23{ }^{\circ} \mathrm{C}$ for $5 \mathrm{~min}$, then was warmed to $60{ }^{\circ} \mathrm{C}$. After $10 \mathrm{~min}$, a solution of the bromoenetriyne 21 (61.5 mg, $84.3 \mu \mathrm{mol}, 1$ equiv) in a 1:1 mixture of THF and pyridine $(30 \mathrm{~mL})$ was added via cannula over $12 \mathrm{~min}$. After $6 \mathrm{~min}$, the reaction mixture was allowed to cool to ambient temperature and the cooled solution was partitioned between a 1:1 mixture of ether and hexanes $(120 \mathrm{~mL}$, cooled in an ice-water bath) and 1.0 M aqueous copper (II) sulfate solution (80 $\mathrm{mL}$ ). The layers were separated and the organic layer was washed with $1.0 \mathrm{M}$ aqueous copper (II) sulfate solution $(2 \times 80 \mathrm{~mL})$. The aqueous layers were combined and the combined solution was extracted with a 1:1 mixture of ether and hexanes $(80 \mathrm{~mL})$. The organic extracts were combined and the combined solution was washed with brine $(30 \mathrm{~mL})$. The washed solution was dried over anhydrous sodium sulfate and toluene $(3 \mathrm{~mL})$ was added. The dried solution was filtered and the filtrate was concentrated to a volume of $\sim 3 \mathrm{~mL}$. The residue was purified by flash-column chromatography (13\% ether-pentane). To determine the yield of the reaction, fractions containing pure 22 were combined and the combined solution $(\sim 50 \mathrm{~mL})$ was diluted with $\mathrm{C}_{6} \mathrm{D}_{6}(50 \mathrm{~mL})$. The solution was concentrated to a volume of ca. $1 \mathrm{~mL}$. A ${ }^{1} \mathrm{H}$ NMR spectrum was taken to confirm purity and that no toluene was present, then an internal standard (toluene, $5.0 \mu \mathrm{L}, 47 \mu \mathrm{mol}$ ) was added. The yield of $\mathbf{2 2}$ was determined by comparing integrated peak areas for $\mathbf{2 2}$ relative to toluene (calculated: $46.0 \mathrm{mg}, 75 \%$ ).

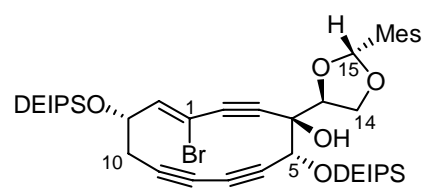

22

$\mathrm{R}_{f}=0.47$ (20\% ether-hexanes). ${ }^{1} \mathrm{H}$ NMR (500 MHz, $\left.\mathrm{C}_{6} \mathrm{D}_{6}\right), \delta 6.68$ (s, 1H, C(15)-H), 6.58 (s, 2H, Ar-H), 6.37 (d, J = 9.0 Hz, 1H, C(12)-H), 4.92 (m, 2H, C(13)-H and C(11)-H), 4.82 (s, 1H, C(5)-H), 4.48 (dd, $\left.\mathrm{J}_{1}=6.6 \mathrm{~Hz}, \mathrm{~J}_{2}=9.0 \mathrm{~Hz}, 1 \mathrm{H}, \mathrm{C}(14)-\mathbf{H}\right), 4.48$ (t, J = 8.4 Hz, 1H, C(14)-H), 2.47 (s, $\left.6 \mathrm{H}, \mathrm{Ar}-\mathrm{CH}_{3}\right), 2.32\left(\mathrm{ddd}, \mathrm{J}_{1}=0.6 \mathrm{~Hz}, \mathrm{~J}_{2}=7.2 \mathrm{~Hz}, \mathrm{~J}_{3}=16.8 \mathrm{~Hz}, 1 \mathrm{H}, \mathrm{C}(10)-\mathbf{H}\right), 2.19$ (ddd, $\mathrm{J}_{1}=1.2 \mathrm{~Hz}$, $\left.\mathrm{J}_{2}=7.2 \mathrm{~Hz}, \mathrm{~J}_{3}=16.8 \mathrm{~Hz}, 1 \mathrm{H}, \mathrm{C}(10)-\mathbf{H}\right), 2.07$ (s, 3H, Ar-CH $\mathbf{H}_{3}, 1.98$ (s, br, 1H, C(4)-OH), 0.98-0.79 $\left(\mathrm{m}, 26 \mathrm{H}, \mathrm{Si}\left(\mathrm{CH}_{2} \mathrm{CH}_{3}\right)_{2} \mathrm{CH}\left(\mathrm{CH}_{3}\right)_{2}\right), 0.59-0.48\left(\mathrm{~m}, 8 \mathrm{H}, \mathrm{Si}\left(\mathrm{CH}_{2} \mathrm{CH}_{3}\right)_{2} \mathrm{CH}\left(\mathrm{CH}_{3}\right)_{2}\right) . \quad{ }^{13} \mathrm{C} \mathrm{NMR}(100$ $\left.\mathrm{MHz}, \mathrm{C}_{6} \mathrm{D}_{6}\right), \delta 142.5,138.5,138.3,130.4,129.7,128.3,128.1,127.9,103.4,103.2,96.7,89.8,89.4$, 87.6, 80.0, 77.7, 74.3 (2), 72.2, 67.8, 29.8, 20.6, 20.0, 17.0, 12.7, 12.6, 6.7 (3), 3.5, 3.4, 3.2 (2). IR $\left(\mathrm{NaCl}\right.$, thin film), $\mathrm{cm}^{-1} 3547,2956,2877,1614,1460,1107,1076,804,733$. HRMS-ESI $(\mathrm{m} / \mathrm{z})$ $[\mathrm{M}+\mathrm{H}]^{+}$calcd for $\mathrm{C}_{38} \mathrm{H}_{56} \mathrm{BrO}_{5} \mathrm{Si}_{2}$, 727.2849; found, 727.2827. 

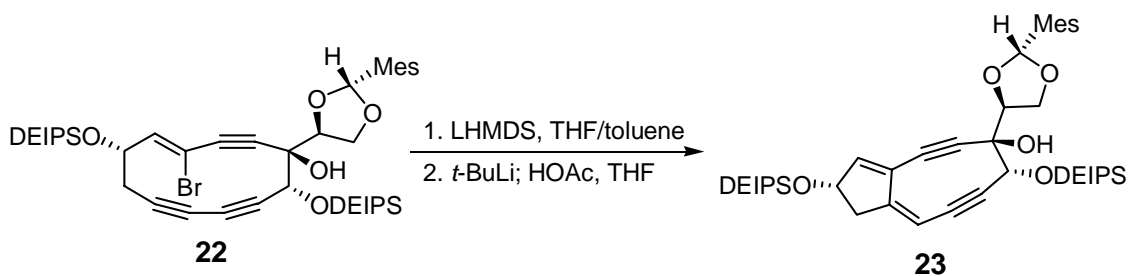

\section{Bicyclic Dienediyne 23}

4- $\AA$ Molecular sieves (crushed, activated, $2 \mathrm{~g}$ ) were added to a solution of the cyclic bromoenetriyne $22(21.8 \mathrm{mg}, 30.0 \mu \mathrm{mol}, 1$ equiv) in a 1:1 mixture of THF and toluene $(10 \mathrm{~mL})$. After stirring at $23{ }^{\circ} \mathrm{C}$ for $15 \mathrm{~min}$, the mixture was cooled to $-78{ }^{\circ} \mathrm{C}$. A solution of lithium hexamethyldisilazide in tetrahydrofuran $(1.0 \mathrm{M}, 33.0 \mu \mathrm{L}, 33.0 \mu \mathrm{mol}, 1.1$ equiv) was added. After $30 \mathrm{sec}$, a solution of tert-butyllithium in pentane $(1.7 \mathrm{M}, 70.6 \mu \mathrm{L}, 0.120 \mathrm{mmol}, 4.0$ equiv) was added to the cold $\left(-78{ }^{\circ} \mathrm{C}\right)$ reaction mixture, followed immediately $(<3 \mathrm{~s})$ by quenching with a solution of acetic acid in THF ( $250 \mu \mathrm{L}$ of a 4:1 mixture of THF and acetic acid, respectively). The reaction mixture was partitioned between a 1:1 mixture of ether and hexanes $(30 \mathrm{~mL})$ and saturated aqueous sodium bicarbonate solution $(20 \mathrm{~mL})$. The aqueous layer was extracted with ether $(20$ $\mathrm{mL}$ ). The organic extracts were combined and the combined solution was washed with brine (10 $\mathrm{mL})$. The washed solution was dried over anhydrous sodium sulfate and toluene $(3 \mathrm{~mL})$ was added. The dried solution was filtered and the filtrate was concentrated to a volume of $\sim 3 \mathrm{~mL}$. The residue was purified by flash-column chromatography (13\% ether-pentane). To determine the yield of the reaction, fractions containing pure $\mathbf{2 3}$ were combined and the combined solution ( $\sim 50$ $\mathrm{mL}$ ) was diluted with $\mathrm{C}_{6} \mathrm{D}_{6}(50 \mathrm{~mL})$. The solution was concentrated to a volume of ca. $1 \mathrm{~mL}$. A ${ }^{1} \mathrm{H}$ NMR spectrum was taken to confirm purity and that no toluene was present, then an internal standard (toluene, $5.0 \mu \mathrm{L}, 47 \mu \mathrm{mol}$ ) was added. The yield of $\mathbf{2 3}$ was determined by comparing integrated peak areas for $\mathbf{2 3}$ relative to toluene (calculated: $5.8 \mathrm{mg}, 30 \%$ ).

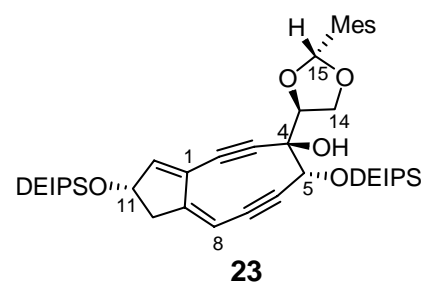

$\mathrm{R}_{f}=0.32$ (20\% ether-hexanes). ${ }^{1} \mathrm{H}$ NMR (500 MHz, CDCN $\left.{ }_{3}\right), \delta 6.81(\mathrm{~s}, 2 \mathrm{H}, \mathrm{Ar}-\mathrm{H}), 6.33(\mathrm{~s}, 1 \mathrm{H}$, C(15)-H), 6.24 (s, br, 1H, C(12)-H), 5.32 (s, br, 1H, C(8)-H), 5.00 (m, 1H, C(11)-H), 4.83 (s, 1H, $\mathrm{C}(5)-\mathrm{H}), 4.60(\mathrm{t}, \mathrm{J}=6.0 \mathrm{~Hz}, 1 \mathrm{H}, \mathrm{C}(13)-\mathrm{H}), 4.33\left(\mathrm{dd}, \mathrm{J}_{1}=7.5 \mathrm{~Hz}, \mathrm{~J}_{2}=6.0 \mathrm{~Hz}, 1 \mathrm{H}, \mathrm{C}(14)-\mathbf{H}\right), 4.13$ (t, $\mathrm{J}=7.0 \mathrm{~Hz}, 1 \mathrm{H}, \mathrm{C}(14)-\mathbf{H}), 4.03(\mathrm{~s}, 1 \mathrm{H}, \mathrm{C}(4)-\mathrm{OH}), 3.01\left(\mathrm{dd}, \mathrm{J}_{1}=5.0 \mathrm{~Hz}, \mathrm{~J}_{2}=14.5 \mathrm{~Hz}, 1 \mathrm{H}, \mathrm{C}(10)-\mathbf{H}\right)$, $2.41(\mathrm{~d}, \mathrm{~J}=14.5 \mathrm{~Hz}, 1 \mathrm{H}, \mathrm{C}(10)-\mathbf{H}), 2.30$ (s, 6H, Ar-CH $\left.\mathbf{C H}_{3}\right), 2.22$ (s, 3H, Ar-CH $\mathrm{CH}_{3}, 1.01-0.92(\mathrm{~m}, 26 \mathrm{H}$, $\left.\mathrm{Si}\left(\mathrm{CH}_{2} \mathrm{CH}_{3}\right)_{2} \mathrm{CH}\left(\mathrm{CH}_{3}\right)_{2}\right), 0.74-0.61$ (m, 8H, $\left.\mathrm{Si}\left(\mathrm{CH}_{2} \mathrm{CH}_{3}\right)_{2} \mathrm{CH}\left(\mathrm{CH}_{3}\right)_{2}\right)$. ${ }^{13} \mathrm{C}$ NMR (100 MHz, $\left.\mathrm{C}_{6} \mathrm{D}_{6}\right), \delta$ 158.3, 143.2, 138.5, 138.4, 130.3, 129.7, 103.6, 102.8, 100.5, 100.3, 92.5, 88.4, 82.3, 78.0, $74.8,74.1,67.7,41.4,20.9,20.2,17.4(2), 13.3,13.1,7.2,7.1,4.0(2), 3.9 . \quad \mathrm{IR}(\mathrm{NaCl}$, thin film), $\mathrm{cm}^{-1} 2956,2876,1462,1083,732$. HRMS-ESI $(\mathrm{m} / z)[\mathrm{M}+\mathrm{H}]^{+}$calcd for $\mathrm{C}_{38} \mathrm{H}_{57} \mathrm{O}_{5} \mathrm{Si}_{2}, 649.3744$; found, 649.3755 . 


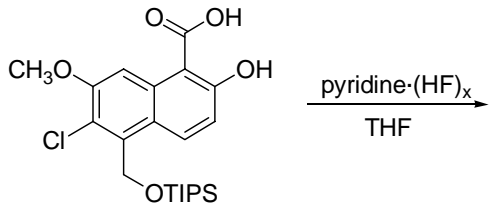

31

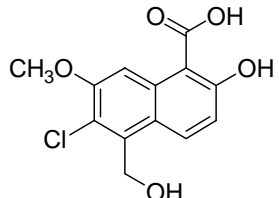

32

\section{2-Hydroxy-1-naphthoic Acid 32}

Hydrogen fluoride-pyridine complex $(2.0 \mathrm{~mL})$ was added to a solution of the naphthoic acid derivative $31^{8}$ (300 mg, $0.683 \mathrm{mmol}, 1$ equiv) in tetrahydrofuran $\left(4 \mathrm{~mL}\right.$ ) at $23{ }^{\circ} \mathrm{C}$. After stirring at $23{ }^{\circ} \mathrm{C}$ for $21 \mathrm{~h}$, the mixture was partitioned between ethyl acetate $(40 \mathrm{~mL})$ and saturated aqueous sodium bicarbonate solution $(40 \mathrm{~mL})$. The aqueous layer was separated and extracted with ethyl acetate $(40 \mathrm{~mL})$. The combined organic layers were washed successively with $2 \mathrm{~N}$ aqueous hydrochloric acid solution $(20 \mathrm{~mL})$, water $(20 \mathrm{~mL})$, and brine $(20 \mathrm{~mL})$. The washed

organic solution was dried over anhydrous sodium sulfate. The dried solution was filtered and the filtrate was concentrated. The residue was washed with hexanes $(10 \mathrm{~mL})$ to give the acid $\mathbf{3 2}$ (white solid, $192 \mathrm{mg}$, 99\%).

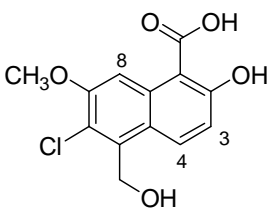

32

${ }^{1} \mathrm{H}$ NMR $\left(500 \mathrm{MHz}, \mathrm{CD}_{3} \mathrm{OD}\right), \delta 8.39$ (s, 1H, C(8)-H), 8.18 (d, J = 9.5 Hz, 1H, C(4)-H), 7.00 (d, J = $9.0 \mathrm{~Hz}, 1 \mathrm{H}, \mathrm{C}(3)-\mathbf{H}), 5.11$ (s, 2H, C(5)-CH $\left.\mathbf{H}_{2} \mathrm{OH}\right), 3.93$ (s, 3H, C(7)-OCH $) .{ }^{13} \mathrm{C}$ NMR (100 MHz, $\left.\mathrm{CD}_{3} \mathrm{OD}\right), \delta 174.8,165.3,155.5,136.3,134.1,133.5,124.1,122.9,118.3,107.2,106.0,59.0,56.3$. IR ( $\mathrm{NaCl}$, thin film) $\mathrm{cm}^{-1} 3446,2954,1631,1610,1416,1228,1111,1005$. HRMS-ESI $(\mathrm{m} / \mathrm{z})$ $[\mathrm{M}-\mathrm{H}]^{-}$calcd for $\mathrm{C}_{13} \mathrm{H}_{10} \mathrm{ClO}_{5} \mathrm{Si}, 281.0217$; found, 281.0230 .
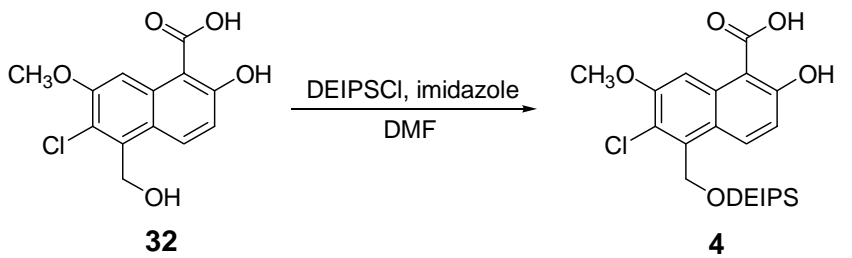

\section{Diethylisopropylsilyl-protected Naphthoic Acid 4}

Diethylisopropylsilyl chloride $(0.252 \mathrm{~mL}, 1.53 \mathrm{mmol}, 4.0$ equiv) was added to a solution of the naphthoic acid 32 (108 mg, $0.382 \mathrm{mmol}, 1$ equiv) and imidazole (156 mg, $2.29 \mathrm{mmol}, 6.0$ equiv) in $N, N$-dimethylformamide $(4 \mathrm{~mL})$ at $23{ }^{\circ} \mathrm{C}$. After stirring at $23{ }^{\circ} \mathrm{C}$ for $17 \mathrm{~h}$, the mixture was partitioned between ethyl acetate $(40 \mathrm{~mL})$ and saturated aqueous sodium bicarbonate solution $(40 \mathrm{~mL})$. The aqueous layer was separated and extracted with ethyl acetate $(40 \mathrm{~mL})$. The combined organic layers were washed successively with $0.25 \mathrm{~N}$ aqueous hydrochloric acid solution $(40 \mathrm{~mL})$, water $(2 \times 15 \mathrm{~mL})$, and brine $(15 \mathrm{~mL})$. The washed organic solution was dried over anhydrous sodium sulfate. The dried solution was filtered and the filtrate was concentrated. The residue was warmed to $40{ }^{\circ} \mathrm{C}$ under high vacuum ( $\sim .1$ Torr) for $2 \mathrm{~h}$ to remove all traces of diethylisopropyl silanol. After cooling, the diethylisopropylsilyl-protected naphthoic acid $\mathbf{4}$ was

\footnotetext{
${ }^{8}$ Ji, N.; Rosen, B. M.; Myers, A. G. Org. Lett. 2003, 6, 4551.
} 
obtained as a white solid (155 mg, 99\%).

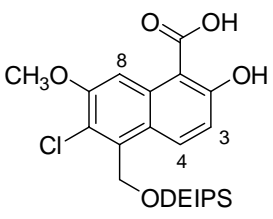

4

${ }^{1} \mathrm{H}$ NMR (400 MHz, CD $\left.{ }_{3} \mathrm{OD}\right), \delta 8.40$ (s, $\left.1 \mathrm{H}, \mathrm{C}(8)-\mathrm{H}\right), 8.17$ (d, J = 9.6 Hz, 1H, C(4)-H), 6.97 (d, J = $9.2 \mathrm{~Hz}, 1 \mathrm{H}, \mathrm{C}(3)-\mathrm{H}), 5.16$ (s, 2H, C(5)-CH $\left.\mathrm{CH}_{2} \mathrm{ODEIPS}\right), 3.93$ (s, 3H , C(7)-OCH $), 0.99-0.91(\mathrm{~m}, 11 \mathrm{H}$, $\left.\mathrm{C}(5)-\mathrm{CH}_{2} \mathrm{OSi}\left(\mathrm{CH}\left(\mathrm{CH}_{3}\right)_{2}\right)\left(\mathrm{CH}_{2} \mathrm{CH}_{3}\right)_{2}\right), \quad 0.67 \quad(\mathrm{t}, \quad \mathrm{J}=8.0 \quad \mathrm{~Hz}, \quad 6 \mathrm{H}$, $\left.\left.\mathrm{C}(5)-\mathrm{CH}_{2} \mathrm{OSi}\left(\mathrm{CH}\left(\mathrm{CH}_{3}\right)_{2}\right)\left(\mathrm{CH}_{2} \mathrm{CH}_{3}\right)_{2}\right)\right) .{ }^{13} \mathrm{C} \mathrm{NMR}\left(100 \mathrm{MHz}, \mathrm{CDCl}_{3}\right), \delta 174.9,165.4,155.4$, 136.3, 134.1 (2), 124.4, 122.1, 118.1, 107.2, 105.9, 60.7, 56.4, 17.7, 14.0, 7.3, 4.3. IR (NaCl, thin film) $\mathrm{cm}^{-1} 2955,2876,1633,1419,1216,1062,725$. HRMS-ESI $(\mathrm{m} / z)[\mathrm{M}-\mathrm{H}]^{-}$calcd for $\mathrm{C}_{20} \mathrm{H}_{27} \mathrm{ClO}_{5} \mathrm{Si}$, 409.1238; found, 409.1222 .

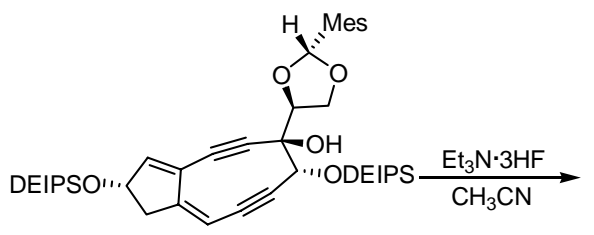

23

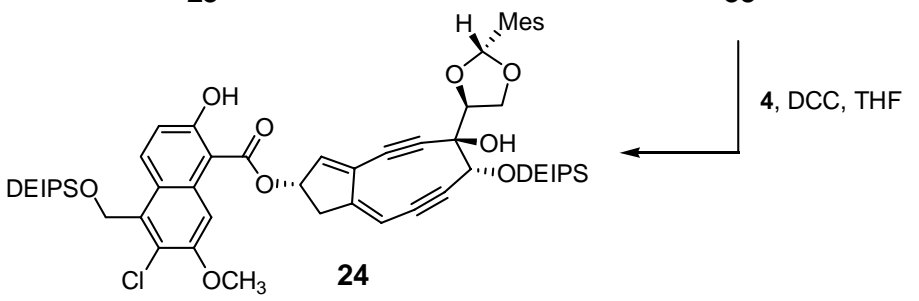

\section{Ester 24}

Triethylamine trihydrofluoride ( $616 \mu \mathrm{L}, 3.78 \mathrm{mmol}, 60$ equiv) was added to a solution of the bicyclic dienediyne 23 (40.9 mg, $63.0 \mu \mathrm{mol}, 1$ equiv) in $\mathrm{CH}_{3} \mathrm{CN}(15 \mathrm{~mL})$ at $-25{ }^{\circ} \mathrm{C}$. After stirring at $-25^{\circ} \mathrm{C}$ for $13 \mathrm{~h}$, the reaction mixture was partitioned between a 1:1 mixture of ether and hexanes $(80 \mathrm{~mL})$ and saturated aqueous sodium bicarbonate solution $(60 \mathrm{~mL})$. The aqueous layer 
was separated and extracted with a 1:1 mixture of ether and hexanes $(60 \mathrm{~mL})$. The organic extracts were combined and the combined solution was washed with brine $(20 \mathrm{~mL})$. The washed organic solution was dried over anhydrous sodium sulfate and toluene $(3 \mathrm{~mL})$ was added. The solution was filtered and the filtrate was concentrated to a volume of $\sim 3 \mathrm{~mL}$. The residue was purified by flash-column chromatography (67\% ether-pentane). Fractions containing 33 were combined and the combined solution was diluted with anhydrous tetrahydrofuran $(100 \mathrm{~mL}){ }^{9} \quad$ The resulting solution was concentrated to a volume of $\sim 10 \mathrm{~mL}$ and the concentrate was cooled in ice.

The naphthoic acid 4 (54.4 $\mathrm{mg}, 0.132 \mathrm{mmol}, 2.1$ equiv) and $N, N^{\prime}$-dicyclohexylcarbodiimide $(72.7 \mathrm{mg}, 0.378 \mathrm{mmol}, 6$ equiv) were added in sequence to the ice-cold solution of the allylic alcohol 33 in tetrahydrofuran $(\sim 10 \mathrm{~mL})$. After stirring at $0{ }^{\circ} \mathrm{C}$ for $2.5 \mathrm{~h}$, the reaction mixture was filtered through a plug of Celite ${ }^{\circledR}$, and the filtrate was diluted with a 1:1 mixture of ether and hexanes $(40 \mathrm{~mL})$. The resulting solution was washed with water $(20 \mathrm{~mL})$. The aqueous layer was separated and extracted with a 1:1 mixture of ether and hexanes $(40 \mathrm{~mL})$. The organic layers were combined and the combined solution was washed with brine $(15 \mathrm{~mL})$. The washed organic solution was dried over anhydrous sodium sulfate and toluene $(3 \mathrm{~mL})$ was added. The residue was purified by flash-column chromatography (33\% ether-pentane). To determine the yield of the reaction, fractions containing pure 24 were combined $(\sim 50 \mathrm{~mL})$ and the combined solution was diluted with $\mathrm{C}_{6} \mathrm{D}_{6}(50 \mathrm{~mL})$. The solution was concentrated to a volume of ca. $1 \mathrm{~mL}$. A ${ }^{1} \mathrm{H}$ NMR spectrum was taken to confirm purity and that no toluene was present, then an internal standard (toluene, $5.0 \mu \mathrm{L}, 47 \mu \mathrm{mol}$ ) was added. The yield of $\mathbf{2 4}$ was determined by comparing integrated peak areas for $\mathbf{2 4}$ relative to toluene (calculated: $25.4 \mathrm{mg}, 44 \%, 2 \mathrm{steps}$ ).

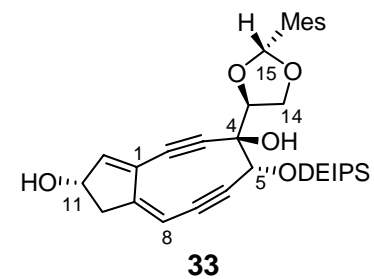

$\mathrm{R}_{f}=0.27$ (66\% ether-hexanes). ${ }^{1} \mathrm{H}$ NMR (500 MHz, $\left.\mathrm{C}_{6} \mathrm{D}_{6}\right), \delta 6.69(\mathrm{~s}, 2 \mathrm{H}, \mathrm{Ar}-\mathbf{H}), 6.66(\mathrm{~s}, 1 \mathrm{H}$, $\mathrm{C}(15)-\mathbf{H}), 5.82$ (m, br, 1H, C(12)-H), 5.00 (s, br, 1H, C(8)-H), 5.00 (t, J = 9.0 Hz, 1H, C(13)-H), $4.91(\mathrm{~s}, 1 \mathrm{H}, \mathrm{C}(5)-\mathbf{H}), 4.57\left(\mathrm{dd}, \mathrm{J}_{1}=9.0 \mathrm{~Hz}, \mathrm{~J}_{2}=6.5 \mathrm{~Hz}, 1 \mathrm{H}, \mathrm{C}(14)-\mathbf{H}\right), 4.46$ (t, J = 8.0 Hz, 1H, $\mathrm{C}(14)-\mathbf{H}), 4.25$ (m, 1H, C(11)-H), $2.46\left(\mathrm{~s}, 6 \mathrm{H}, \operatorname{Ar}-\mathrm{CH}_{3}\right), 2.36\left(\mathrm{dd}, \mathrm{J}_{1}=8.0 \mathrm{~Hz}, \mathrm{~J}_{2}=20.0 \mathrm{~Hz}, 1 \mathrm{H}\right.$, C(10)-H), 2.35 (s, br, 1H, C(4)-OH), 2.07 (s, 3H, Ar-CH $\mathbf{H}_{3}, 1.98$ (d, J = 20.0 Hz, 1H, C(10)-H), 1.06-0.96 (m, 13H, $\left.\mathrm{Si}\left(\mathrm{CH}_{2} \mathrm{CH}_{3}\right)_{2} \mathrm{CH}\left(\mathrm{CH}_{3}\right)_{2}\right), \quad 0.76-0.59 \quad\left(\mathrm{~m}, \quad 4 \mathrm{H}, \quad \mathrm{Si}\left(\mathrm{CH}_{2} \mathrm{CH}_{3}\right)_{2} \mathrm{CH}\left(\mathrm{CH}_{3}\right)_{2}\right)$. HRMS-ESI $(\mathrm{m} / \mathrm{z})[\mathrm{M}+\mathrm{H}]^{+}$calcd for $\mathrm{C}_{31} \mathrm{H}_{41} \mathrm{O}_{5} \mathrm{Si}, 521.2723$; found, 521.2737.

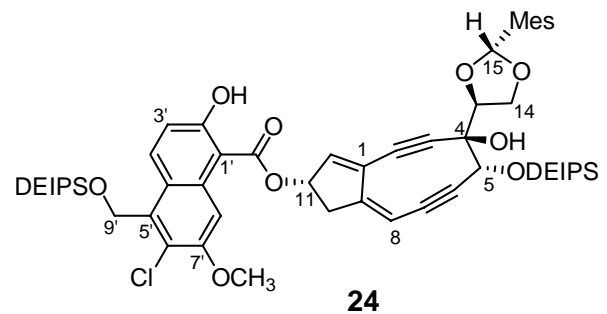

\footnotetext{
${ }^{9}$ For analytical purposes, in a separate experiment pooled column fractions of pure intermediate $\mathbf{3 3}$ were diluted with $\mathrm{C}_{6} \mathrm{D}_{6}(\sim 50 \mathrm{~mL})$, the resulting solution was partially concentrated $(\sim 1 \mathrm{~mL})$, and a ${ }^{1} \mathrm{H}$ NMR spectrum was recorded, providing the data tabulated below and reproduced as part of this Supporting Information.
} 
$\mathrm{R}_{f}=0.25$ (33\% ether-hexanes). ${ }^{1} \mathrm{H}$ NMR (500 MHz, $\left.\mathrm{C}_{6} \mathrm{D}_{6}\right), \delta 12.72\left(\mathrm{~s}, 1 \mathrm{H}, \mathrm{C}\left(2^{\prime}\right)-\mathrm{OH}\right), 8.43$ (d, J $\left.=9.5 \mathrm{~Hz}, 1 \mathrm{H}, \mathrm{C}\left(4^{\prime}\right)-\mathbf{H}\right), 8.06\left(\mathrm{~s}, 1 \mathrm{H}, \mathrm{C}\left(8^{\prime}\right)-\mathbf{H}\right), 7.19$ (d, J = 9.5 Hz, 1H, C(3')-H), 6.70 (s, 2H, Ar-H), 6.57 (s, 1H, C(15)-H), 5.88 (s, br, 1H, C(12)-H), $5.50(\mathrm{~m}, 1 \mathrm{H}, \mathrm{C}(11)-\mathrm{H}), 5.24$ (d, J = $2.5 \mathrm{~Hz}, 2 \mathrm{H}$, C(9')-H), 4.98 (s, br, 1H, C(8)-H), 4.90 (t, J = 6.5 Hz, 1H, C(13)-H), 4.85 (s, 1H, C(5)-H), 4.54 (dd, $\left.\mathrm{J}_{1}=9.0 \mathrm{~Hz}, \mathrm{~J}_{2}=6.5 \mathrm{~Hz}, 1 \mathrm{H}, \mathrm{C}(14)-\mathbf{H}\right), 4.38(\mathrm{~d}, \mathrm{~J}=9.0 \mathrm{~Hz}, 1 \mathrm{H}, \mathrm{C}(14)-\mathbf{H}), 3.55(\mathrm{~s}, 3 \mathrm{H}$, $\left.\mathrm{C}\left(7^{\prime}\right)-\mathrm{OCH}_{3}\right), 2.47$ (m, 1H, C(10)-H), 2.42 (s, 6H, Ar-CH $\left.\mathbf{H}_{3}\right), 2.27$ (s, 1H, C(4)-OH), 2.13 (d, J = $15.0 \mathrm{~Hz}, 1 \mathrm{H}, \mathrm{C}(10)-\mathbf{H}), 2.09$ (s, 3H, Ar- $\left.\mathrm{CH}_{3}\right), 1.03-0.95$ (m, 26H, $\left.\mathrm{Si}\left(\mathrm{CH}_{2} \mathrm{CH}_{3}\right)_{2} \mathrm{CH}\left(\mathrm{CH}_{3}\right)_{2}\right)$, 0.72-0.57 (m, 8H, Si $\left.\left(\mathrm{CH}_{2} \mathrm{CH}_{3}\right)_{2} \mathrm{CH}\left(\mathrm{CH}_{3}\right)_{2}\right) .{ }^{13} \mathrm{C}$ NMR (100 MHz, $\left.\mathrm{C}_{6} \mathrm{D}_{6}\right), \delta$ 171.8, 165.8, 156.5, 154.9, 138.6, 138.3, 136.5, 136.3, 134.9, 132.8, 131.8, 130.4, 129.4, 128.5, 123.9, 121.6, 117.7, 106.5, 104.7, 104.4, 103.6, 102.5, 101.5, 91.7, 86.7, 82.2, 78.1, 77.2, 74.4, 67.4, 60.1, 55.6, 37.4, 20.9, 20.1, 17.4(2), 13.2, 13.1, 7.2(2), 3.9(2), 3.8. IR (NaCl, thin film), $\mathrm{cm}^{-1} 2956,2877,1646$, $1613,1464,1420,1203,1113,816$. HRMS-ESI $(\mathrm{m} / z)[\mathrm{M}+\mathrm{H}]^{+}$calcd for $\mathrm{C}_{51} \mathrm{H}_{66} \mathrm{ClO}_{9} \mathrm{Si}_{2}, 913.3934$; found, 913.3900 .
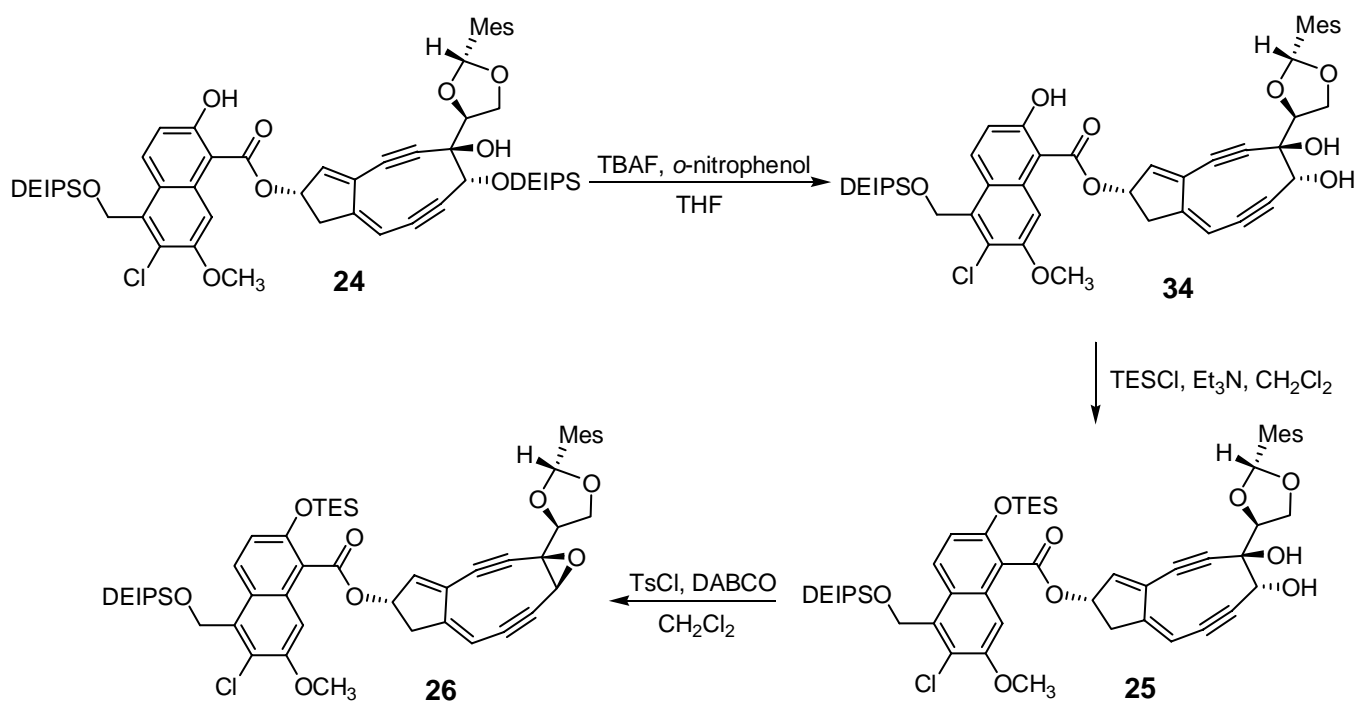

Epoxide 26

A solution of tetrabutylammonium fluoride in tetrahydrofuran $(1.0 \mathrm{M}, 432 \mu \mathrm{L}, 0.432$ mmol, 6.0 equiv) was added to a solution of the ester $24(65.7 \mathrm{mg}, 72.0 \mu \mathrm{mol}, 1$ equiv) and $o$-nitrophenol $\left(60.1 \mathrm{mg}, 0.432 \mathrm{mmol}, 6.0\right.$ equiv) in THF $(25 \mathrm{~mL})$ at $0{ }^{\circ} \mathrm{C}$. The reaction mixture was stirred at $0{ }^{\circ} \mathrm{C}$ for $90 \mathrm{~min}$, then was diluted with a 1:1 mixture of ether and hexanes $(80 \mathrm{~mL})$. The mixture was washed with saturated aqueous ammonium chloride solution $(60 \mathrm{~mL})$. The aqueous layer was separated and extracted with a 1:1 mixture of ether and hexanes $(80 \mathrm{~mL})$. The organic extracts were combined and the combined solution was washed with brine $(30 \mathrm{~mL})$. The washed solution was dried over sodium sulfate and toluene $(3 \mathrm{~mL})$ was added. The dried solution was filtered and the filtrate was concentrated to a volume of $\sim 3 \mathrm{~mL}$. The residue was purified by flash-column chromatography (50\% ether-pentane, grading to $67 \%$ ether-pentane). Fractions containing pure $\mathbf{3 4}$ were combined and the combined solution was diluted with anhydrous dichloromethane $(200 \mathrm{~mL}) .{ }^{10} \quad$ The resulting solution was concentrated to a volume of $\sim 15 \mathrm{~mL}$ and the concentrate was cooled to $-78^{\circ} \mathrm{C}$.

\footnotetext{
${ }^{10}$ For analytical purposes, in a separate experiment pooled column fractions of pure intermediate $\mathbf{3 4}$ were diluted with $\mathrm{C}_{6} \mathrm{D}_{6}(\sim 50 \mathrm{~mL})$, the resulting solution was partially concentrated $(\sim 1 \mathrm{~mL})$, and a ${ }^{1} \mathrm{H}$ NMR spectrum was recorded, providing the data tabulated below and reproduced as part of this Supporting Information.
} 
Triethylamine $(113 \mu \mathrm{L}, 0.810 \mathrm{mmol}, 11$ equiv) and chlorotriethylsilane $(54.3 \mu \mathrm{L}, 0.324$ mmol, 4.5 equiv) were added in sequence to the cold $\left(-78{ }^{\circ} \mathrm{C}\right)$ solution of the triol 34 in dichloromethane $(\sim 15 \mathrm{~mL})$. After stirring at $-78{ }^{\circ} \mathrm{C}$ for $40 \mathrm{~min}$, the reaction mixture was partitioned between a 1:1 mixture of ether and hexanes $(80 \mathrm{~mL})$ and brine $(40 \mathrm{~mL})$. The layers were separated and the aqueous layer was extracted with a 1:1 mixture of ether and hexanes (40 $\mathrm{mL}$ ). The organic extracts were combined and the combined solution was dried over anhydrous sodium sulfate. Toluene $(3 \mathrm{~mL})$ was added. The dried solution was filtered and the filtrate was concentrated to a volume of $\sim 3 \mathrm{~mL}$. The residue was purified by flash-column chromatography (67\% ether-pentane). Fractions containing the diol 25 were combined and the combined solution was diluted with anhydrous dichloromethane $(200 \mathrm{~mL}){ }^{11}$ The resulting solution was concentrated to a volume of $\sim 12 \mathrm{~mL}$.

DABCO (48.5 mg, $0.432 \mathrm{mmol}, 6.0$ equiv) and $p$-toluenesulfonyl chloride (41.2 mg, 0.216 mmol, 3.0 equiv) were added in sequence to the solution of the diol 25 in dichloromethane $(\sim 12 \mathrm{~mL})$ at $23{ }^{\circ} \mathrm{C}$. After stirring at $23{ }^{\circ} \mathrm{C}$ for $110 \mathrm{~min}$, the reaction mixture was diluted with a $1: 1$ mixture of ether and hexanes $(80 \mathrm{~mL})$. The resulting mixture was washed with brine $(40 \mathrm{~mL})$ and the layers were separated. The aqueous layer was extracted with a 1:1 mixture of ether and hexanes $(80 \mathrm{~mL})$. The organic extracts were combined and the combined solution was dried over anhydrous sodium sulfate. Toluene $(4 \mathrm{~mL})$ was added to the dried solution, and the resulting solution was concentrated to a volume of $\sim 4 \mathrm{~mL}$. The residue was purified by flash chromatography ( $17 \%$ ether-pentane, grading to $25 \%$ ether-pentane). To determine the yield of the reaction, fractions containing pure $\mathbf{2 6}$ were combined and the combined solution $(\sim 50 \mathrm{~mL})$ was diluted with $\mathrm{C}_{6} \mathrm{D}_{6}(50 \mathrm{~mL})$. The solution was concentrated to a volume of ca. $1 \mathrm{~mL}$. A ${ }^{1} \mathrm{H}$ NMR spectrum was taken to confirm purity and that no toluene was present, then an internal standard (toluene, $5.0 \mu \mathrm{L}, 47 \mu \mathrm{mol}$ ) was added. The yield of $\mathbf{2 6}$ was determined by comparing integrated peak areas for $\mathbf{2 6}$ relative to toluene (calculated: $20.8 \mathrm{mg}, 33 \%$ over 3 steps).

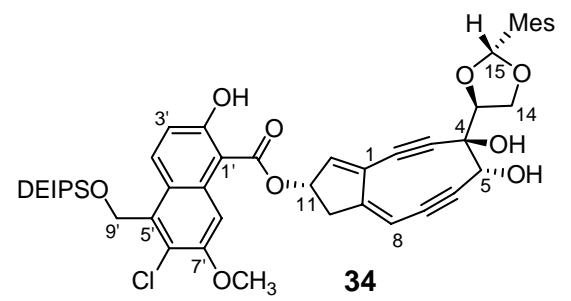

$\mathrm{R}_{f}=0.33$ (66\% ether-hexanes). ${ }^{1} \mathrm{H}$ NMR (500 MHz, $\left.\mathrm{C}_{6} \mathrm{D}_{6}\right), \delta 12.64$ (s, br, $\left.1 \mathrm{H}, \mathrm{C}\left(2^{\prime}\right)-\mathrm{OH}\right), 8.43$ (d, $\left.\mathrm{J}=9.0 \mathrm{~Hz}, 1 \mathrm{H}, \mathrm{C}\left(4^{\prime}\right)-\mathbf{H}\right), 8.07$ (s, 1H, C(8')-H), 7.19 (d, J = 9.5 Hz, 1H, C(3')-H), 6.68 (s, 2H, Ar-H), 6.55 (s, 1H, C(15)-H), 5.95 (t, J = 2.0 Hz, 1H, C(12)-H), 5.53 (m, 1H, C(11)-H), 5.23 (s, $2 \mathrm{H}$, C(9')-H), 4.98 (s, br, 1H, C(8)-H), 4.56 (dd, J J $\left._{1}=8.0 \mathrm{~Hz}, \mathrm{~J}_{2}=7.0 \mathrm{~Hz}, 1 \mathrm{H}, \mathrm{C}(13)-\mathbf{H}\right), 4.38$ (s, $1 \mathrm{H}$, $\mathrm{C}(5)-\mathbf{H}), 4.34\left(\mathrm{dd}, \mathrm{J}_{1}=9.0 \mathrm{~Hz}, \mathrm{~J}_{2}=6.5 \mathrm{~Hz}, 1 \mathrm{H}, \mathrm{C}(14)-\mathbf{H}\right), 4.18$ (d, J = 8.5 Hz, 1H, C(14)-H), 3.57 (s, $\left.3 \mathrm{H}, \mathrm{C}\left(7^{\prime}\right)-\mathrm{OCH}_{3}\right), 2.46\left(\mathrm{ddd}, \mathrm{J}_{1}=18.5 \mathrm{~Hz}, \mathrm{~J}_{2}=8.5 \mathrm{~Hz}, \mathrm{~J}_{3}=2.0 \mathrm{~Hz}, 1 \mathrm{H}, \mathrm{C}(10)-\mathbf{H}\right), 2.36(\mathrm{~s}, 6 \mathrm{H}$, Ar-CH $\left.\mathbf{H}_{3}\right), 2.16(\mathrm{~d}, \mathrm{~J}=18.0 \mathrm{~Hz}, 1 \mathrm{H}, \mathrm{C}(10)-\mathbf{H}), 2.07$ (s, 3H, Ar-CH $\mathbf{H}_{3}, 1.01-0.89$ (m, 13H, $\left.\mathrm{Si}\left(\mathrm{CH}_{2} \mathrm{CH}_{3}\right)_{2} \mathrm{CH}\left(\mathrm{CH}_{3}\right)_{2}\right), 0.70-0.59\left(\mathrm{~m}, 4 \mathrm{H}, \mathrm{Si}\left(\mathrm{CH}_{2} \mathrm{CH}_{3}\right)_{2} \mathrm{CH}\left(\mathrm{CH}_{3}\right)_{2}\right)$. HRMS-ESI $(\mathrm{m} / z)[\mathrm{M}+\mathrm{H}]^{+}$ calcd for $\mathrm{C}_{44} \mathrm{H}_{49} \mathrm{ClO}_{9} \mathrm{Si}$, 785.2912; found, 785.2947.

\footnotetext{
${ }^{11}$ For analytical purposes, in a separate experiment pooled column fractions of pure intermediate $\mathbf{2 5}$ were diluted with $\mathrm{C}_{6} \mathrm{D}_{6}(\sim 50 \mathrm{~mL})$, the resulting solution was partially concentrated $(\sim 1 \mathrm{~mL})$, and a ${ }^{1} \mathrm{H}$ NMR spectrum was recorded, providing the data tabulated below and reproduced as part of this Supporting Information.
} 


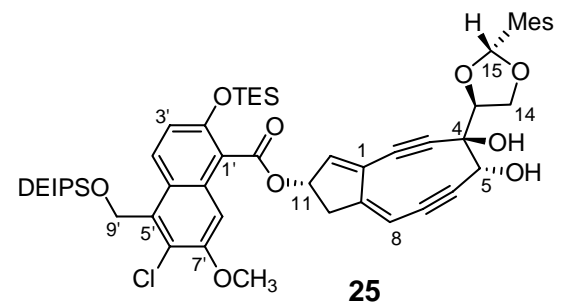

$\mathrm{R}_{f}=0.37$ (66\% ether-hexanes). ${ }^{1} \mathrm{H}$ NMR (500 MHz, $\left.\mathrm{C}_{6} \mathrm{D}_{6}\right), \delta 8.36\left(\mathrm{~d}, \mathrm{~J}=9.5 \mathrm{~Hz}, 1 \mathrm{H}, \mathrm{C}\left(4^{\prime}\right)-\mathbf{H}\right)$, 7.18 (s, 1H, C( $\left.\left.8^{\prime}\right)-\mathbf{H}\right), 7.01$ (d, J = 9.5 Hz, 1H, C(3')-H), 6.68 (s, 2H, Ar-H), 6.56 (s, 1H, C(15)-H), 6.47 (t, J = 2.5 Hz, 1H, C(12)-H), 5.88 (m, 1H, C(11)-H), 5.28 (s, 2H, C(9')-H), 5.02 (t, J = 2.0 Hz, $1 \mathrm{H}, \mathrm{C}(8)-\mathbf{H}), 4.55$ (dd, $\left.\mathrm{J}_{1}=7.5 \mathrm{~Hz}, \mathrm{~J}_{2}=6.0 \mathrm{~Hz}, 1 \mathrm{H}, \mathrm{C}(13)-\mathbf{H}\right), 4.40$ (s, br, $\left.1 \mathrm{H}, \mathrm{C}(5)-\mathbf{H}\right), 4.34$ (dd, $\mathrm{J}_{1}$ $\left.=8.5 \mathrm{~Hz}, \mathrm{~J}_{2}=7.0 \mathrm{~Hz}, 1 \mathrm{H}, \mathrm{C}(14)-\mathbf{H}\right), 4.18(\mathrm{t}, \mathrm{J}=8.5 \mathrm{~Hz}, 1 \mathrm{H}, \mathrm{C}(14)-\mathbf{H}), 3.46\left(\mathrm{~s}, 3 \mathrm{H}, \mathrm{C}\left(7^{\prime}\right)-\mathrm{OCH}_{3}\right)$, 2.66 (ddd, $\left.\mathrm{J}_{1}=20.5 \mathrm{~Hz}, \mathrm{~J}_{2}=7.0 \mathrm{~Hz}, \mathrm{~J}_{3}=2.0 \mathrm{~Hz}, 1 \mathrm{H}, \mathrm{C}(10)-\mathbf{H}\right), 2.54$ (d, J = $\left.18.5 \mathrm{~Hz}, 1 \mathrm{H}, \mathrm{C}(10)-\mathbf{H}\right)$, 2.37 (s, 6H, Ar- $\left.\mathrm{CH}_{3}\right), 2.07$ (s, 3H, Ar- $\left.\mathrm{CH}_{3}\right), 1.05-0.75\left(\mathrm{~m}, 26 \mathrm{H}, \mathrm{Si}\left(\mathrm{CH}_{2} \mathrm{CH}_{3}\right)_{2}\left(\mathrm{CH}\left(\mathrm{CH}_{3}\right)_{2}\right)\right.$ and $\left.\mathrm{Si}\left(\mathrm{CH}_{2} \mathrm{CH}_{3}\right)_{3}\right), 0.72\left(\mathrm{q}, \mathrm{J}=8.0 \mathrm{~Hz}, 4 \mathrm{H}, \mathrm{Si}\left(\mathrm{CH}_{2} \mathrm{CH}_{3}\right)_{2}\right)$. HRMS-ESI $(\mathrm{m} / \mathrm{z})[\mathrm{M}+\mathrm{H}]^{+}$calcd for $\mathrm{C}_{50} \mathrm{H}_{64} \mathrm{ClO}_{9} \mathrm{Si}_{2}, 899.3777$; found, 899.3773.

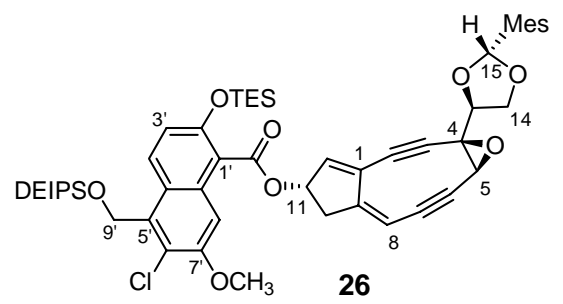

$\mathrm{R}_{f}=0.25$ (25\% ether-hexanes). ${ }^{1} \mathrm{H}$ NMR (500 MHz, $\left.\mathrm{CD}_{3} \mathrm{CN}\right), \delta 8.19\left(\mathrm{~d}, \mathrm{~J}=9.0 \mathrm{~Hz}, 1 \mathrm{H}, \mathrm{C}\left(4^{\prime}\right)-\mathbf{H}\right)$, 7.10 (d, J = $\left.9.5 \mathrm{~Hz}, 1 \mathrm{H}, \mathrm{C}\left(3^{\prime}\right)-\mathbf{H}\right), 7.05$ (s, 1H, C(8')-H), 6.83 (s, 2H, Ar-H), 6.77 (s, br, 1H, C(12)-H), 6.28 (s, 1H, C(15)-H), 5.50 (d, J = 7.0 Hz, br, 1H, C(11)-H), 5.54 (s, br, 1H, C(8)-H), 5.29 (s, 2H, C(9')-H), 4.49 (dd, J $\left.\mathrm{J}_{1}=7.0 \mathrm{~Hz}, \mathrm{~J}_{2}=8.5 \mathrm{~Hz}, 1 \mathrm{H}, \mathrm{C}(13)-\mathrm{H}\right), 4.36(\mathrm{t}, \mathrm{J}=7.0 \mathrm{~Hz}, 1 \mathrm{H}$, C(14)-H), 3.96 (s, 1H, C(5)-H), 3.96 (m, 1H, C(14)-H), 3.91 (s, 3H, C(7')-OCH ${ }_{3}$ ), 3.30 (dd, br, J $\left.=13.5 \mathrm{~Hz}, \mathrm{~J}_{2}=7.0 \mathrm{~Hz}, 1 \mathrm{H}, \mathrm{C}(10)-\mathbf{H}\right), 2.85(\mathrm{~d}, \mathrm{~J}=13.5 \mathrm{~Hz}, 1 \mathrm{H}, \mathrm{C}(10)-\mathbf{H}), 2.33$ (s, 6H, Ar-CH $\mathbf{H}_{3}$, 2.33 (s, 3H, Ar-CH $\left.\mathbf{H}_{3}\right), 1.00-0.64\left(\mathrm{~m}, 26 \mathrm{H}, \mathrm{Si}\left(\mathrm{CH}_{2} \mathrm{CH}_{3}\right)_{2}\left(\mathbf{C H}\left(\mathrm{CH}_{3}\right)_{2}\right)\right.$ and $\left.\mathrm{Si}\left(\mathrm{CH}_{2} \mathrm{CH}_{3}\right)_{3}\right), 0.52$ (q, J = $\left.8.0 \mathrm{~Hz}, 4 \mathrm{H}, \mathrm{Si}\left(\mathrm{CH}_{2} \mathrm{CH}_{3}\right)_{2}\right)$. IR ( $\mathrm{NaCl}$, thin film) $\mathrm{cm}^{-1} 3631,3539,2954,2882,1728,1613,1272$, $1210,1113,858,735$. HRMS-ESI $(\mathrm{m} / \mathrm{z})[\mathrm{M}+\mathrm{H}]^{+}$calcd for $\mathrm{C}_{50} \mathrm{H}_{62} \mathrm{ClO}_{8} \mathrm{Si}_{2}, 881.3672$; found, 881.3677.

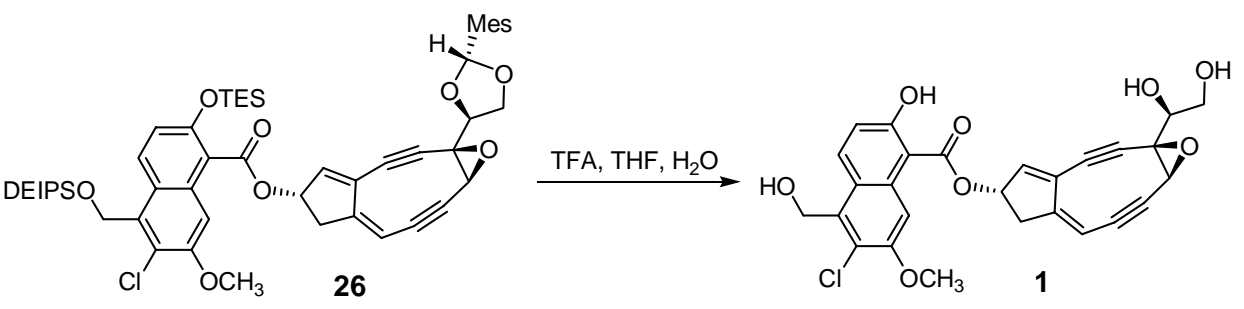

\section{N1999A2 (1)}

Cold, dry trifluoroacetic acid $(180 \mu \mathrm{L}$, cooled in an ice-water bath for at least 30 min prior to use) was added dropwise over $2 \mathrm{~min}$ to an ice-cold solution of the epoxide 26 (10.4 $\mathrm{mg}, 11.8$ $\mu$ mol, 1 equiv) in tetrahydrofuran $(2.6 \mathrm{~mL})$ and water $(0.9 \mathrm{~mL})$. After stirring at $0{ }^{\circ} \mathrm{C}$ for $5 \mathrm{~h}$, the reaction mixture was partitioned between $\mathrm{pH} 7$ aqueous phosphate buffer $(30 \mathrm{~mL})$ and ether $(30$ $\mathrm{mL})$. The layers were separated and the aqueous layer was extracted with ether $(30 \mathrm{~mL})$. The 
organic layers were combined and the combined solution was dried over anhydrous sodium sulfate. The dried solution was concentrated to a volume of ca. $8 \mathrm{~mL}$ and pentane $(5 \mathrm{~mL})$ was added, leading to precipitation of N1999A2 as a yellowish solid. The solid was filtered and washed with $80 \%$ ether-pentane $(3 \times 10 \mathrm{~mL})$ to provide pure N1999A2 $(\mathbf{1})(4.7 \mathrm{mg}, 76 \%)$.

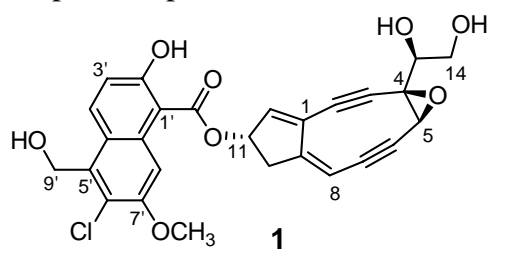

$\mathrm{R}_{f}=0.20$ (100\% ether). ${ }^{1} \mathrm{H}$ NMR (500 MHz, 50\% CD $\left.{ }_{3} \mathrm{CN}-\mathrm{DMSO}-\mathrm{d}_{6}\right), \delta 10.82\left(\mathrm{~s}, 1 \mathrm{H}, \mathrm{C}\left(2^{\prime}\right)-\mathrm{OH}\right)$, 8.22 (d, J = 9.5 Hz, 1H, C(4')-H), 7.43 (s, 1H, C(8')-H), 7.13 (d, J = 9.5 Hz, 1H, C(3')-H), 6.81 (s, br, 1H, C(12)-H), 6.12 (d, J = 7.0 Hz, br, 1H, C(11)-H), 5.59 (s, br, 1H, C(8)-H), 5.31 (d, J = 4.5 Hz, 1H, C(13)-OH), 5.15 (t, J = 5.0 Hz, C(9')-OH), 5.06 (d, J = 5.0 Hz, 2H, C(9')-H), 4.63 (s, br, 1H, C(14)-OH), 3.94 (s, 1H, C(5)-H), 3.93 (s, 3H, C(7')-OH), 3.63-3.55 (m, 3H, C(13)-H + C(14)-H), 3.29 (ddd, $\left.\mathrm{J}_{1}=18 \mathrm{~Hz}, \mathrm{~J}_{2}=7.0 \mathrm{~Hz}, \mathrm{~J}_{3}=2.0 \mathrm{~Hz}, 1 \mathrm{H}, \mathrm{C}(10)-\mathbf{H}\right), 2.94$ (d, J = $\left.18.5 \mathrm{~Hz}, 1 \mathrm{H}, \mathrm{C}(10)-\mathbf{H}\right)$. IR ( $\mathrm{NaCl}$, thin film) $\mathrm{cm}^{-1} 3628,3539,2964,2934,2872,1723,1638,1205,1119$. HRMS-ESI $(\mathrm{m} / \mathrm{z})\left[\mathrm{M}+\mathrm{NH}_{4}\right]^{+}$calcd for $\mathrm{C}_{27} \mathrm{H}_{25} \mathrm{ClO}_{8}, 526.1269$; found, 526.1286 . 


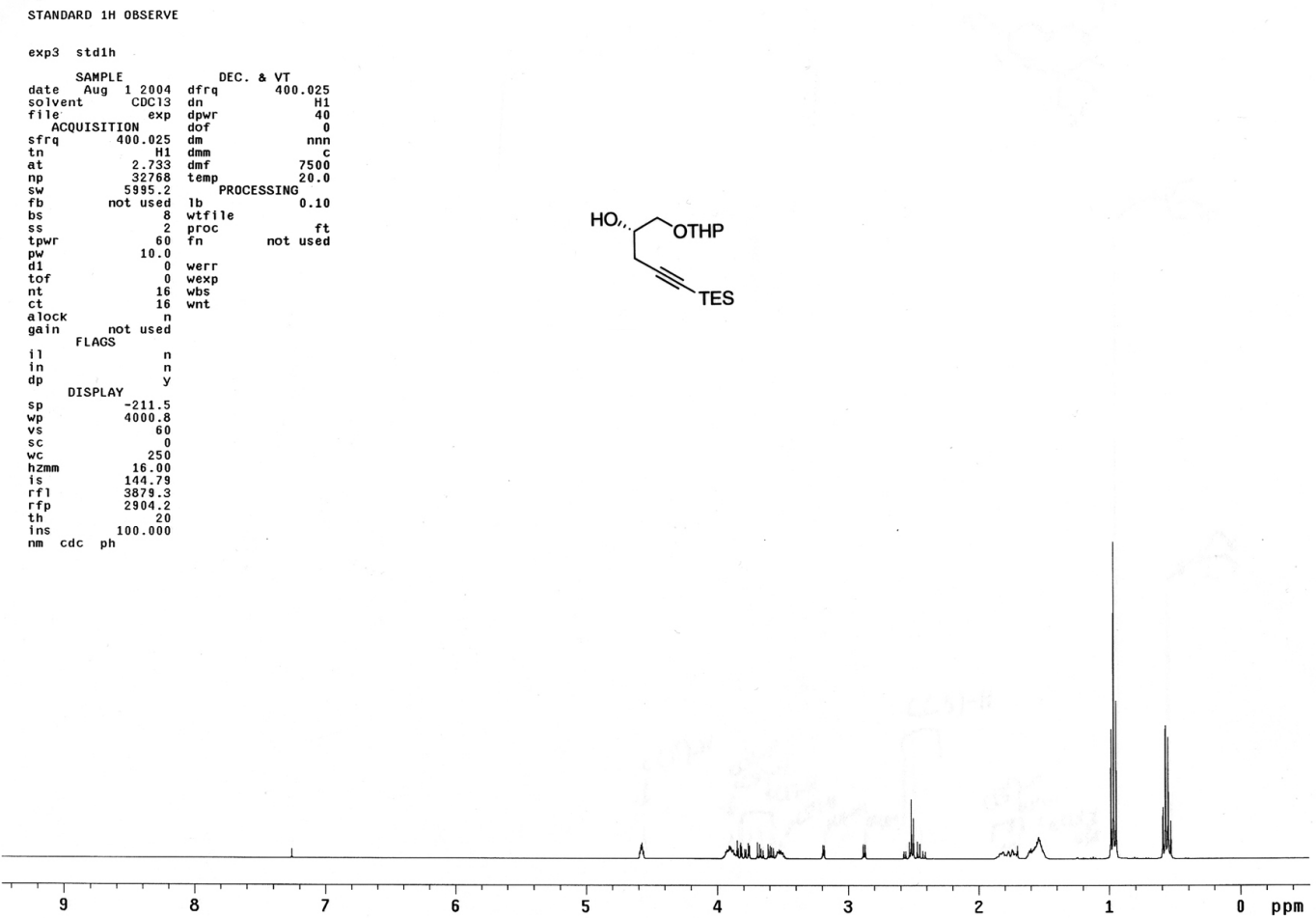



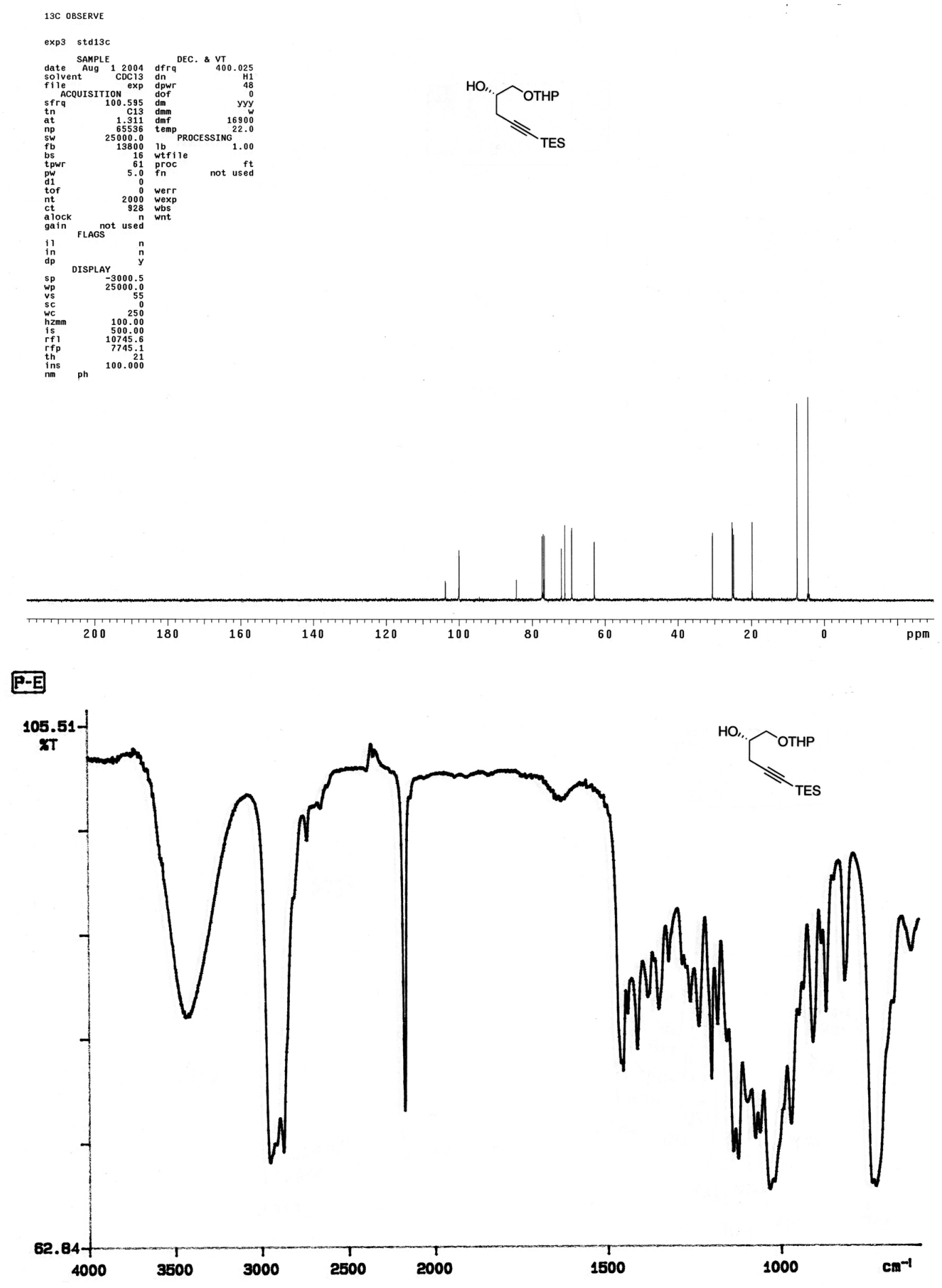
STANDARD PROTON PARAMETERS

exp3 PROTON

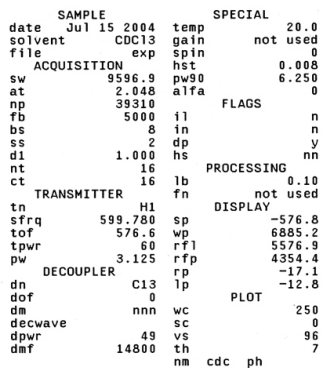
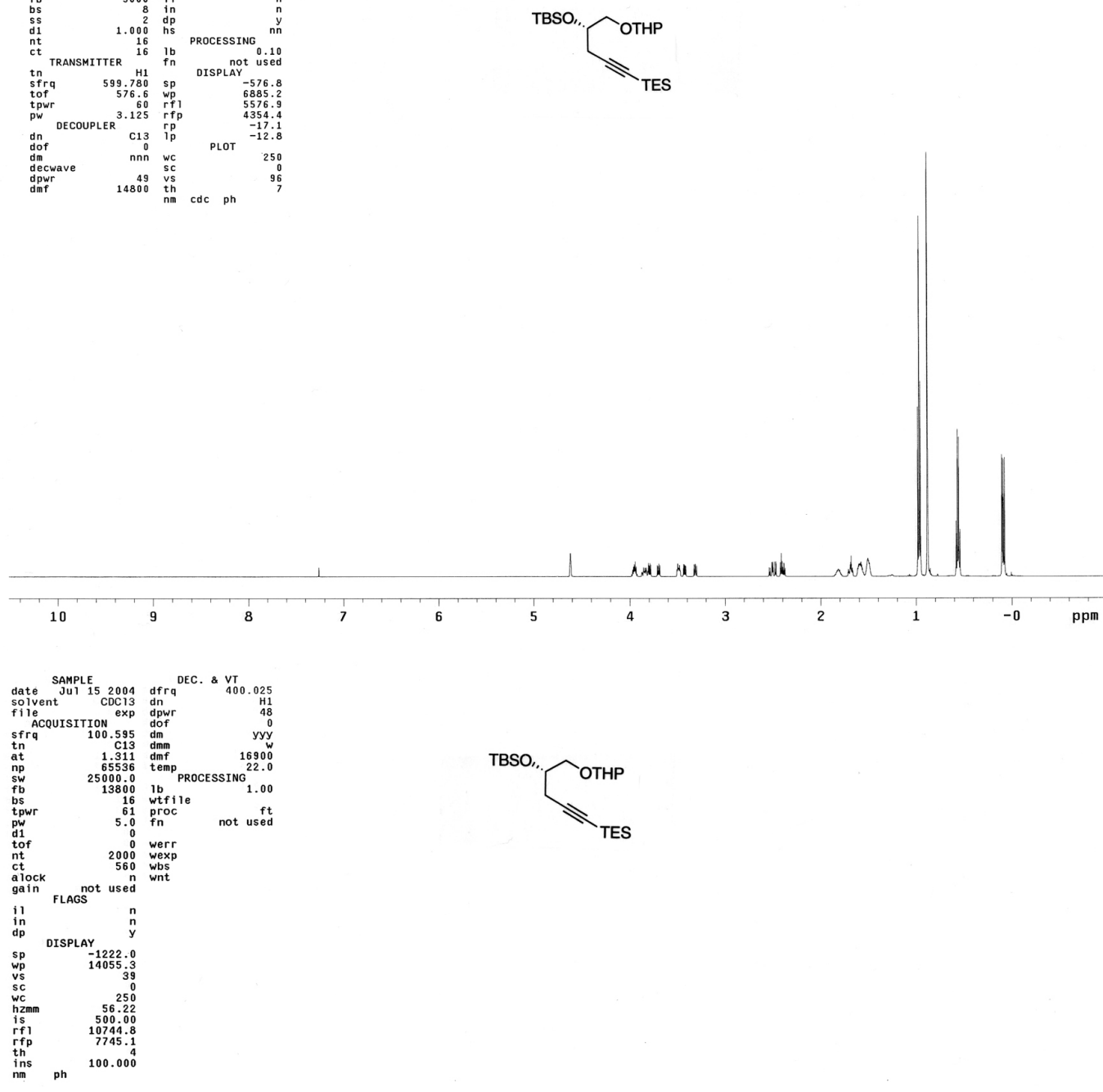

TBSO,

L nith h
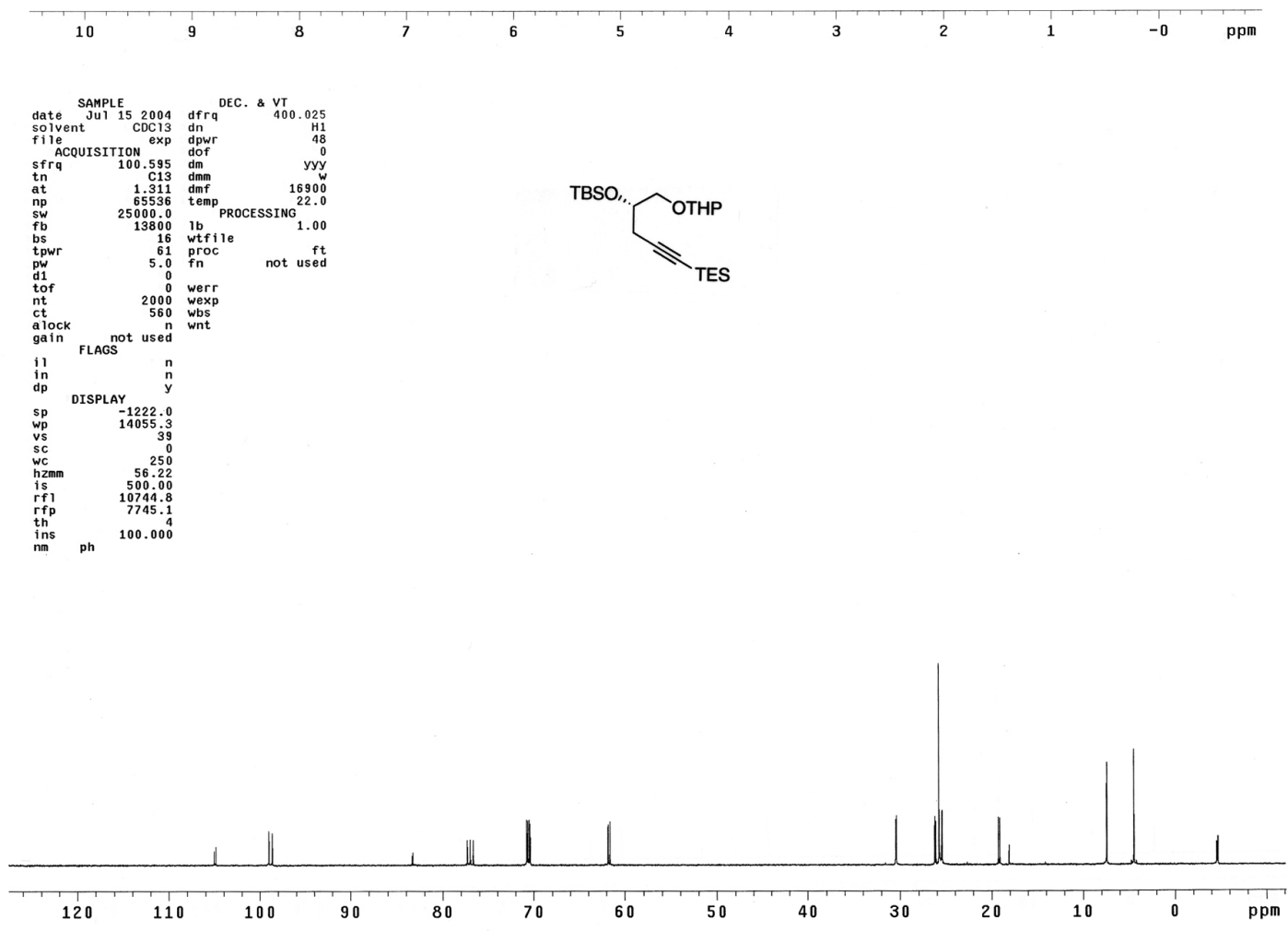

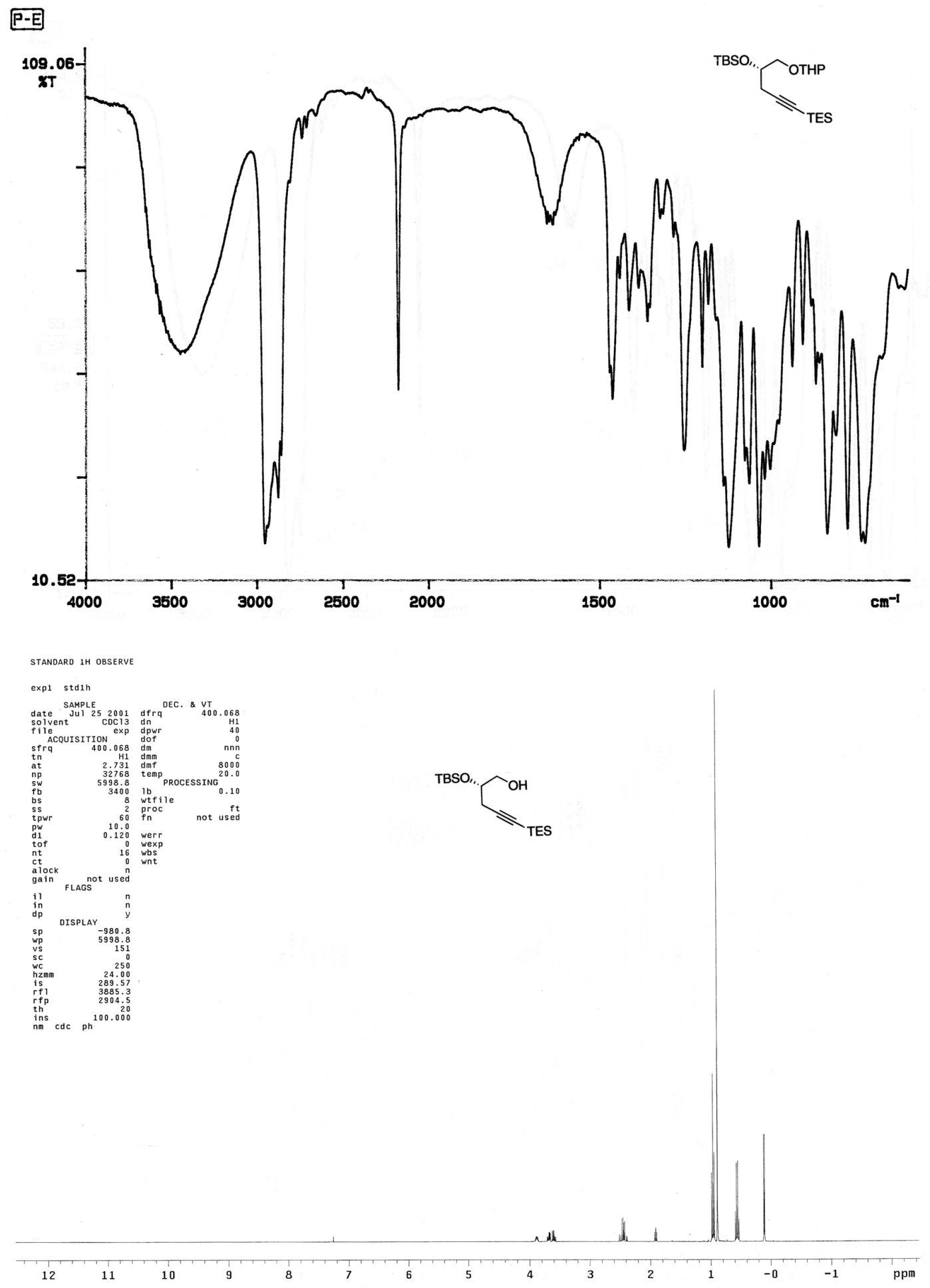

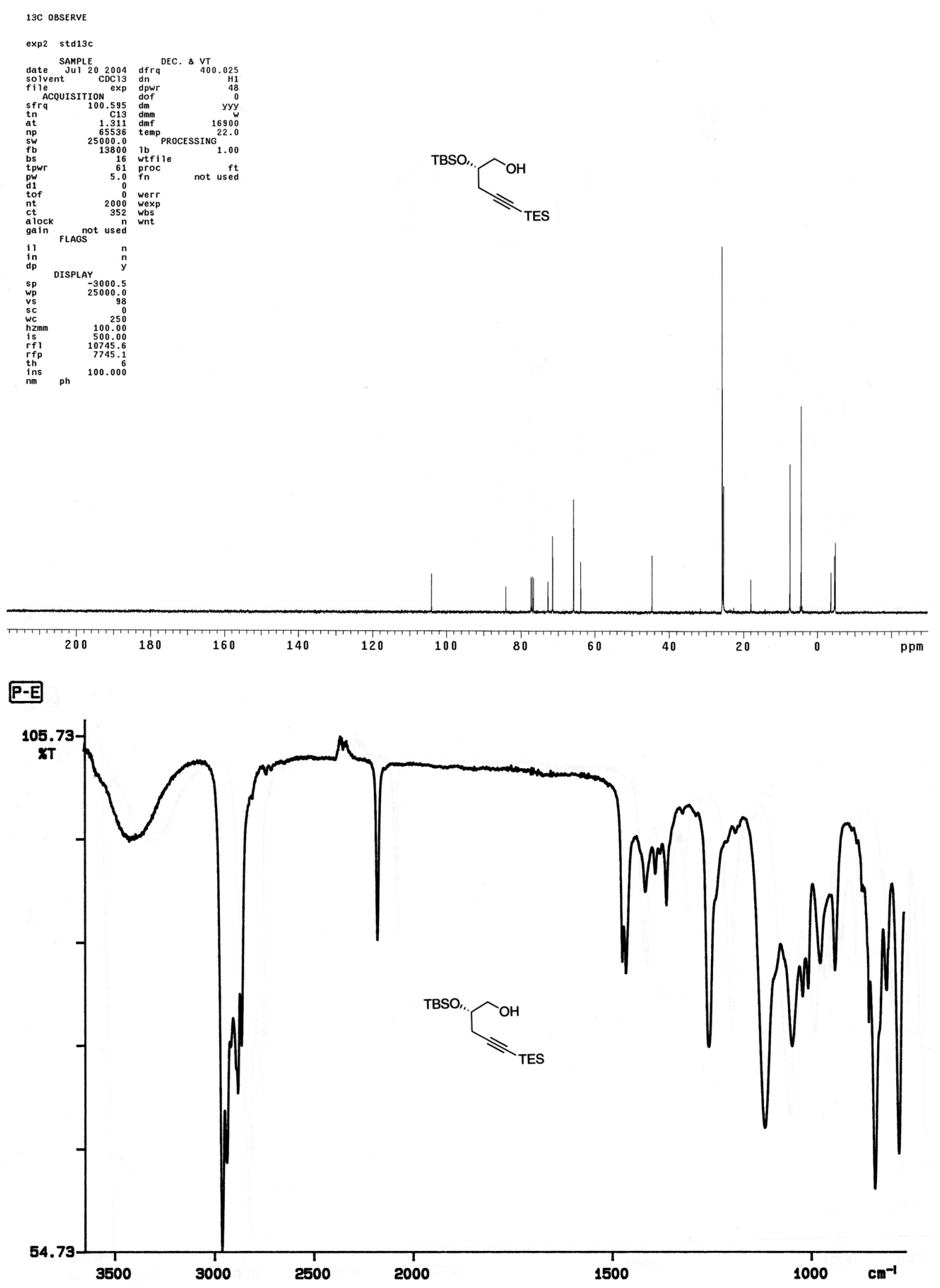

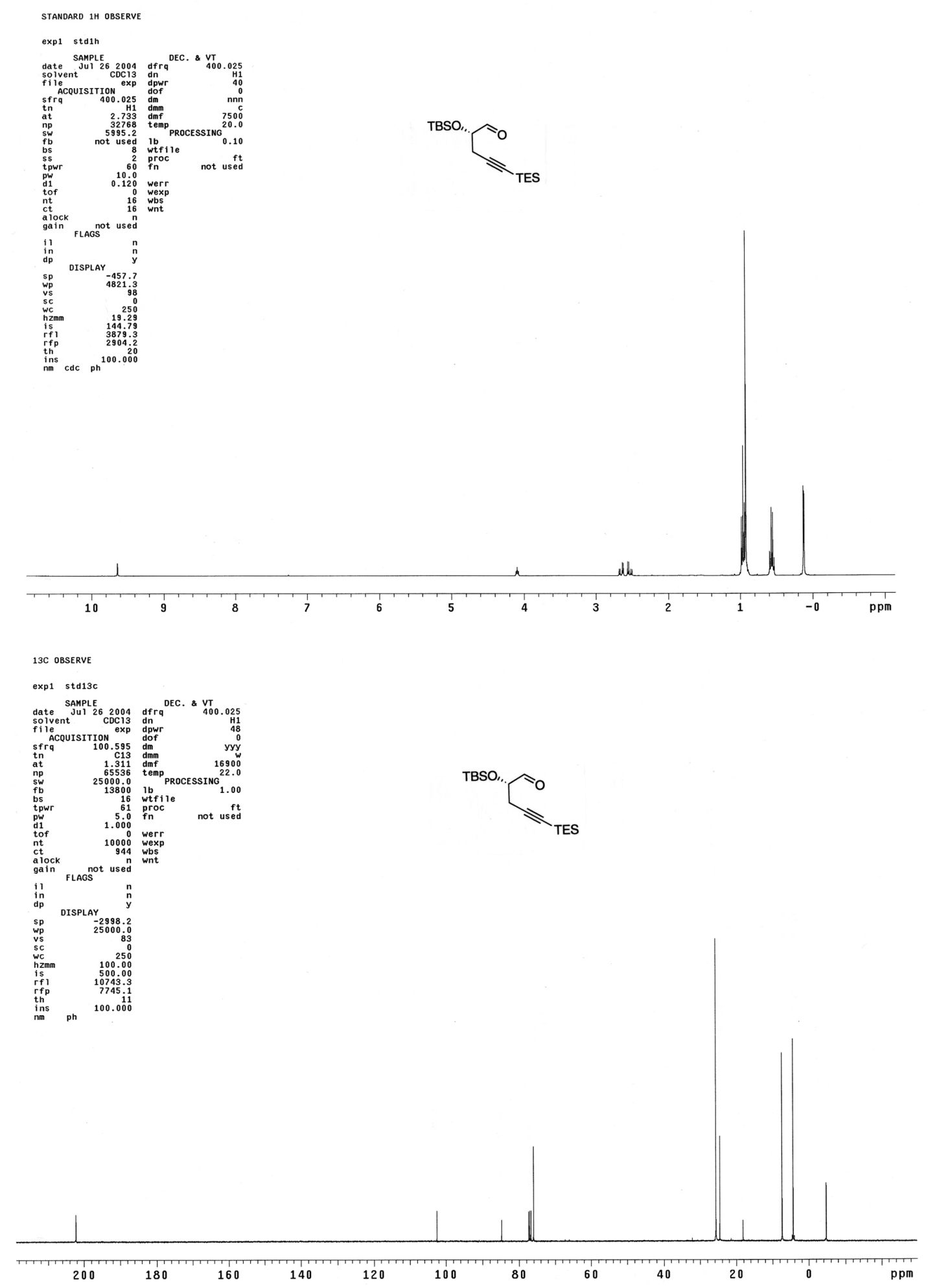


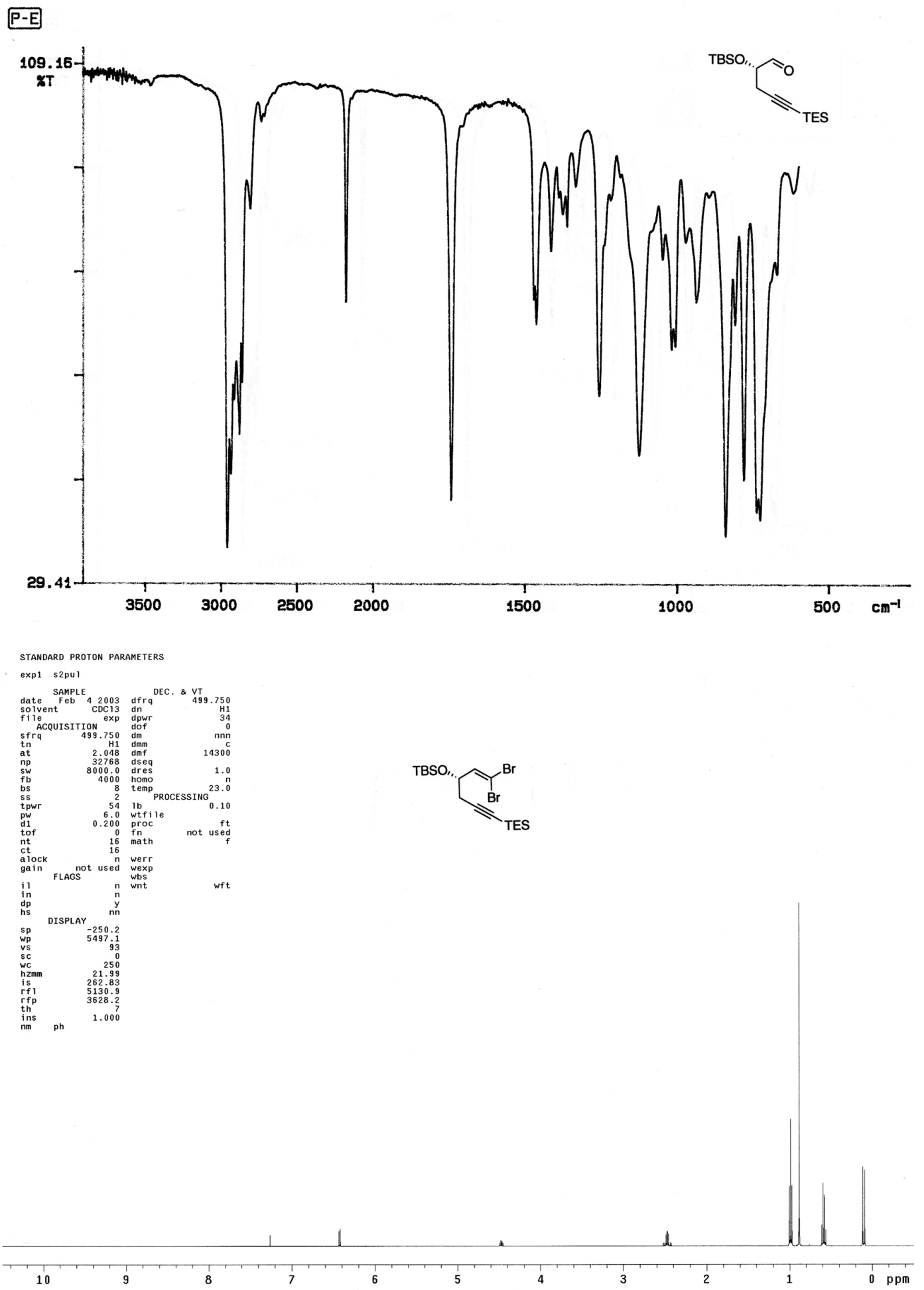



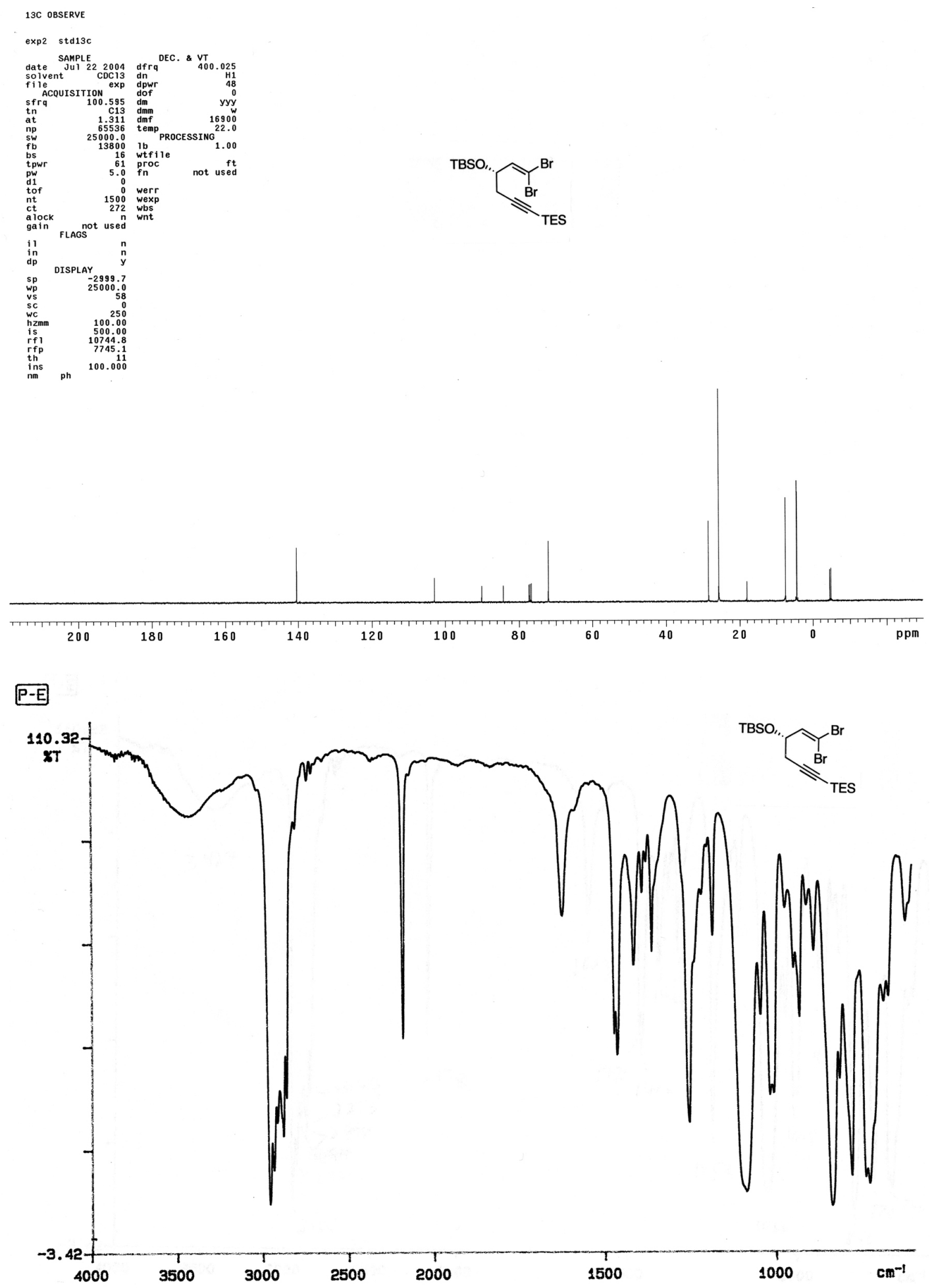

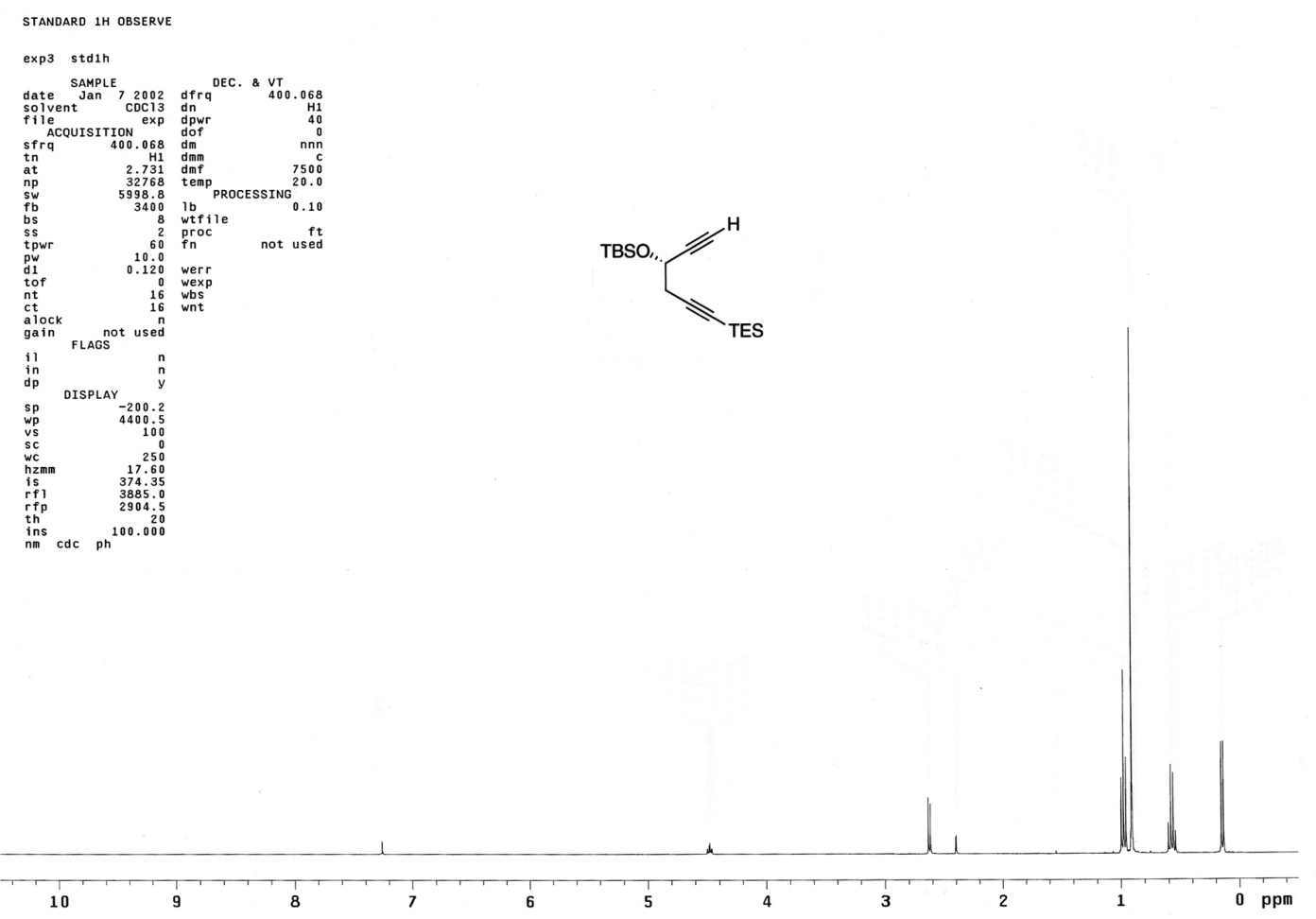

13C OBSERVE

exp3 std13c
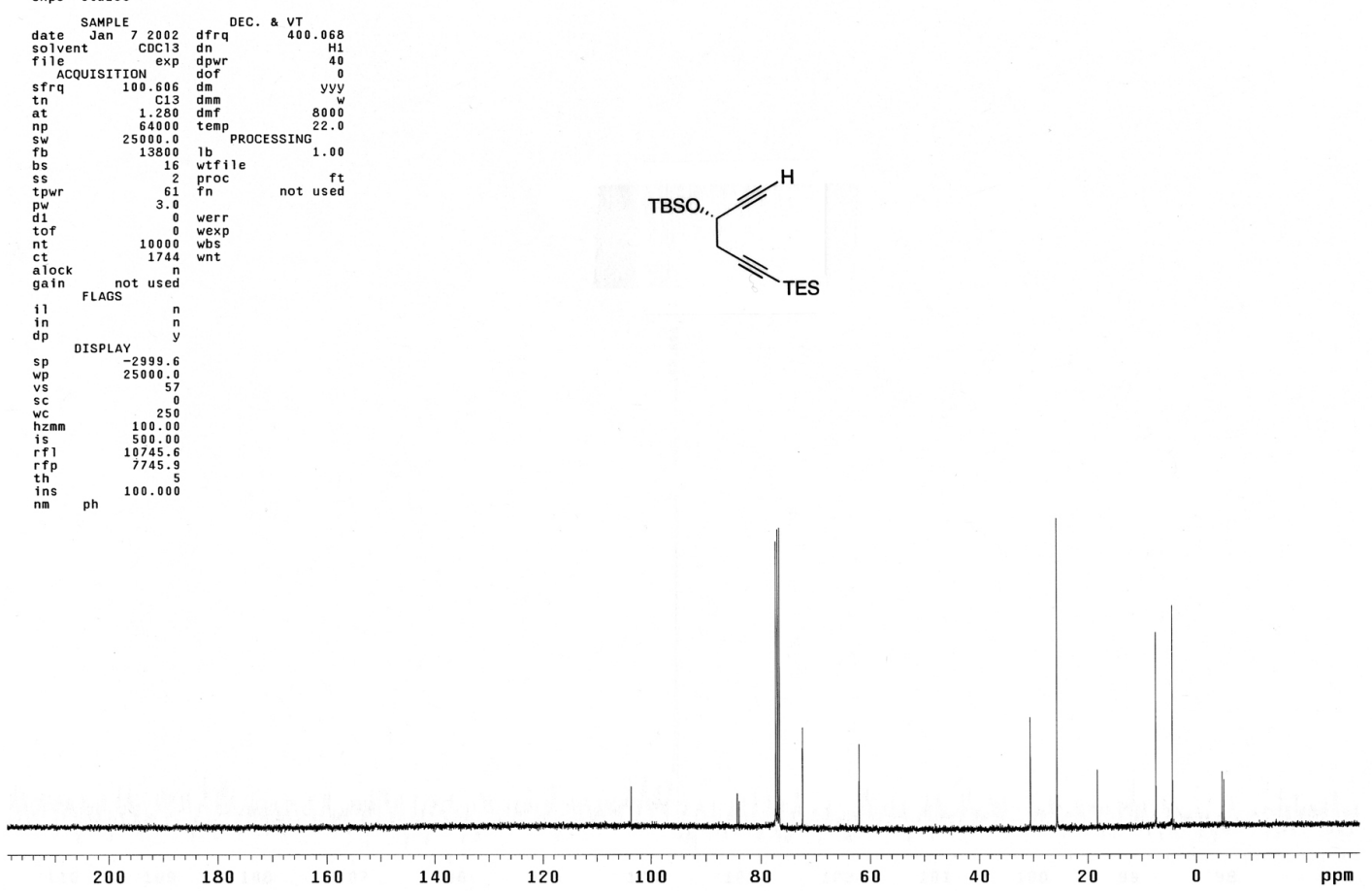


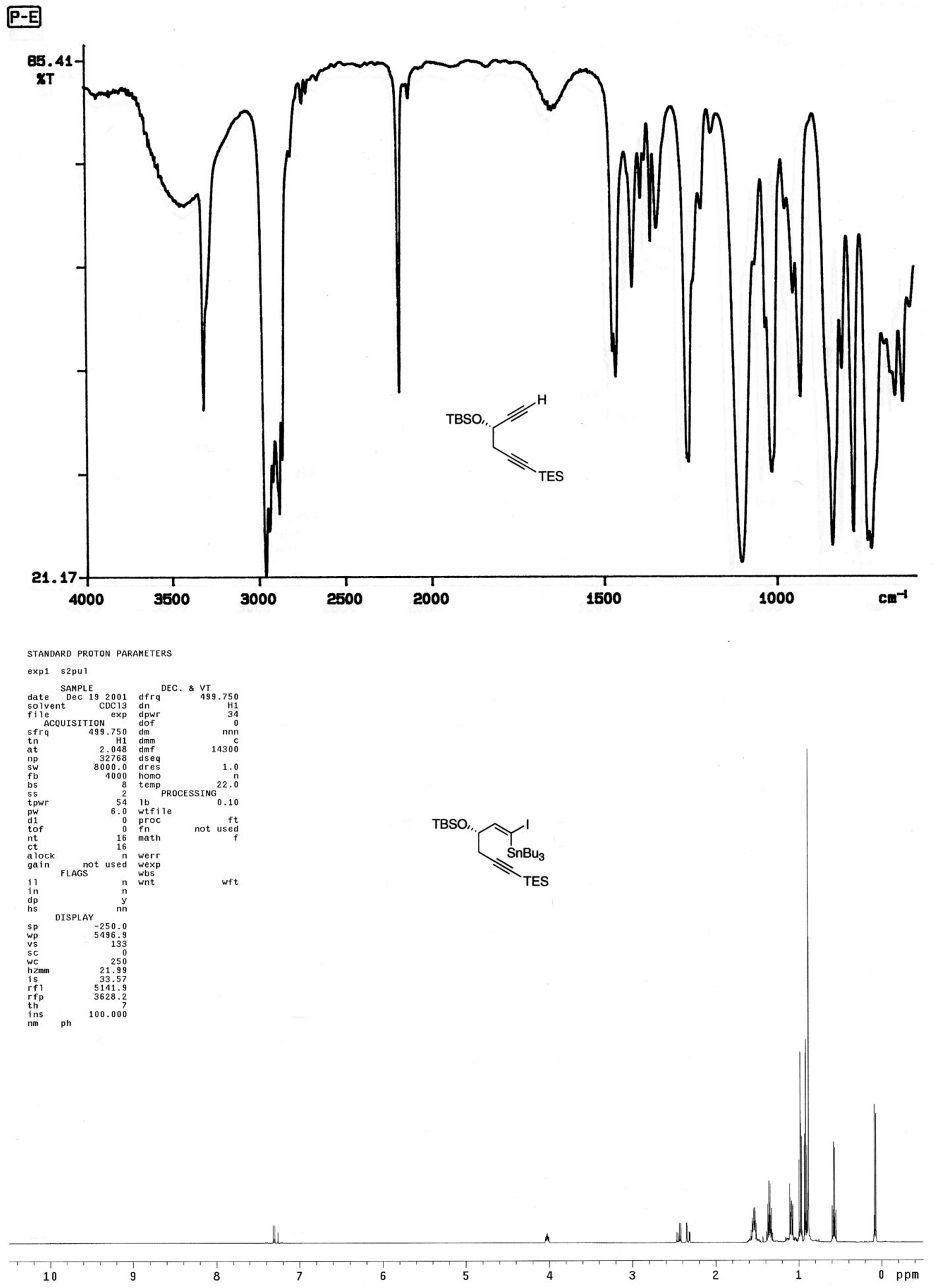



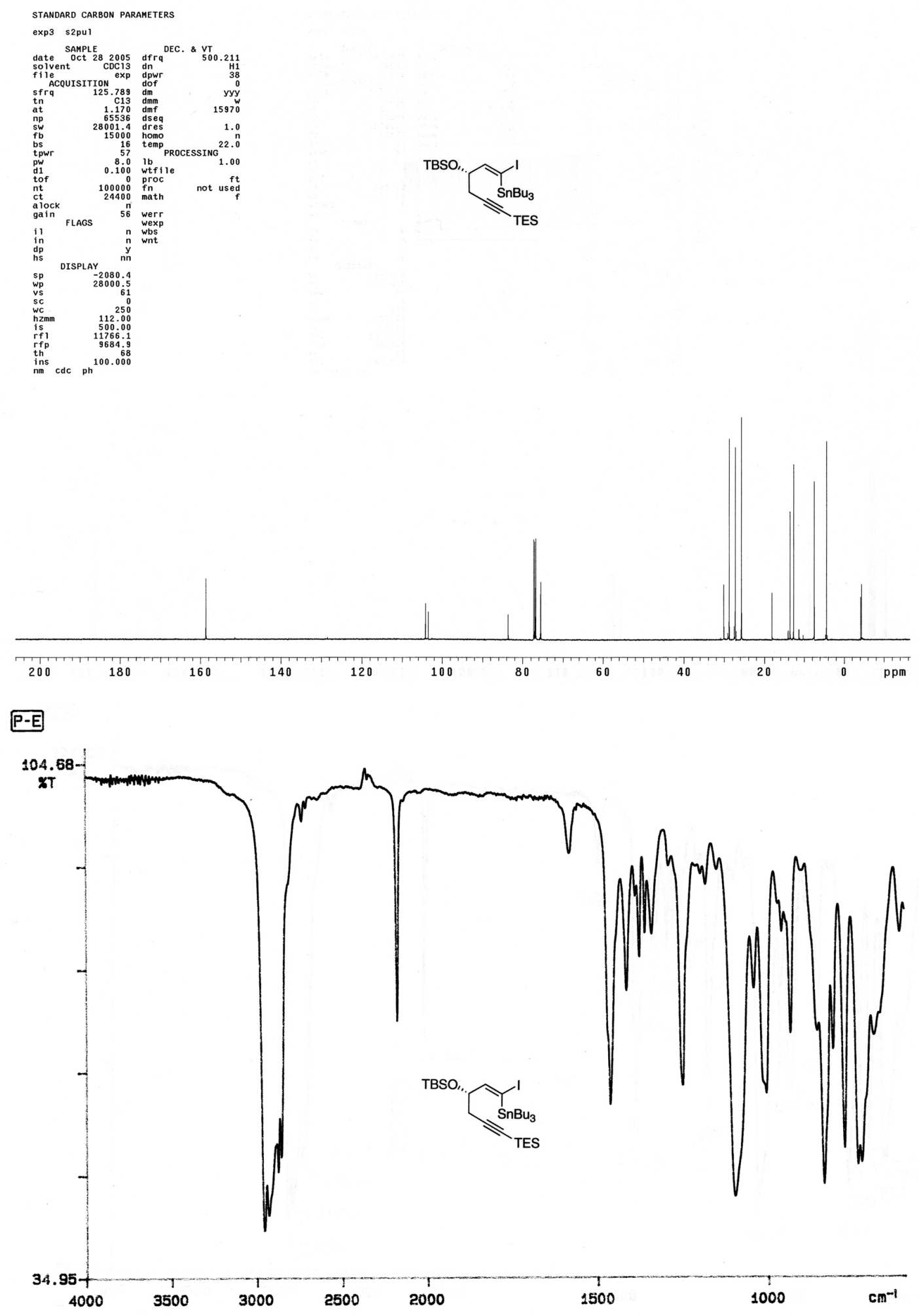
STANDARD PROTON PARAMETERS

exp2 s2pul
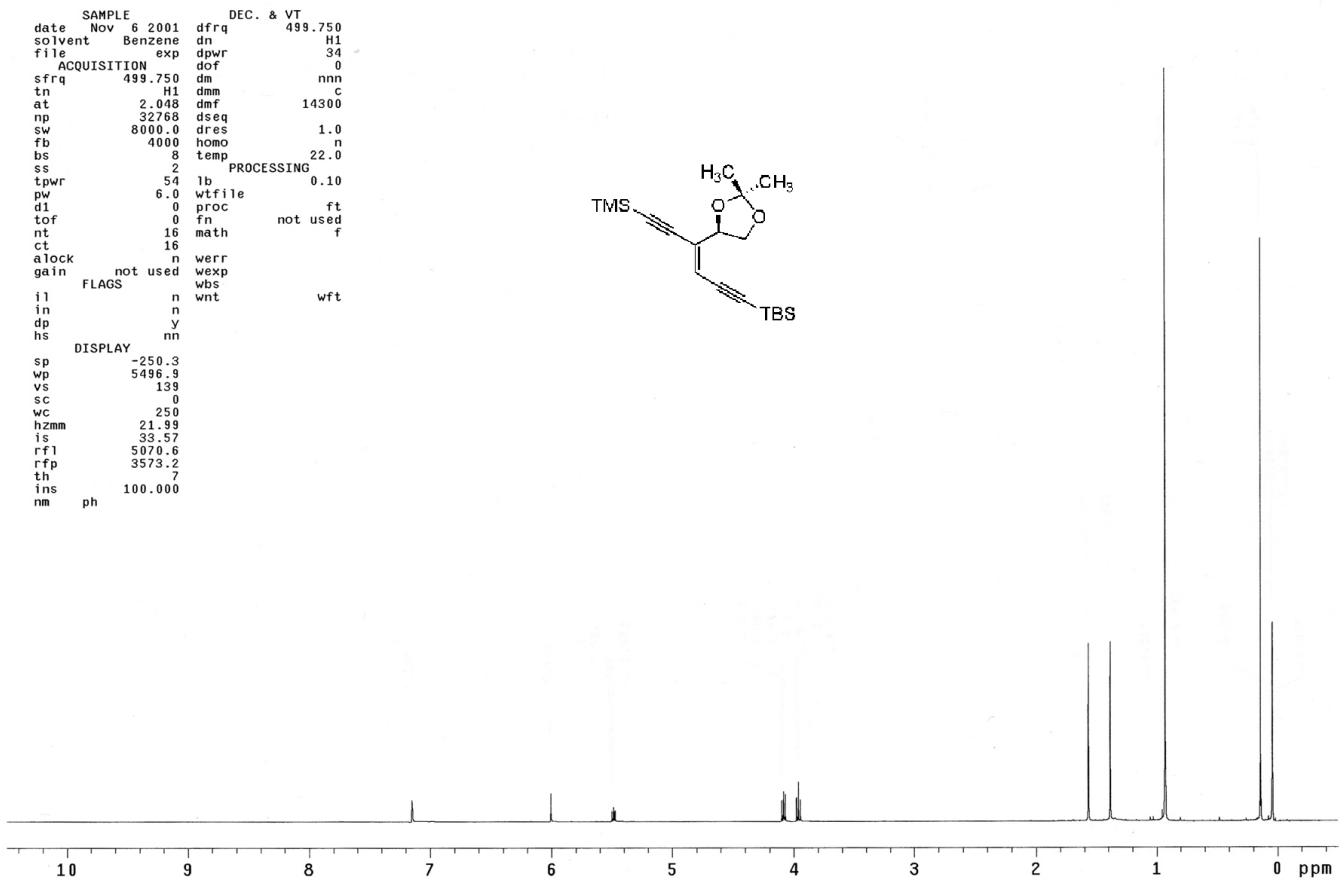

exp2 std13c
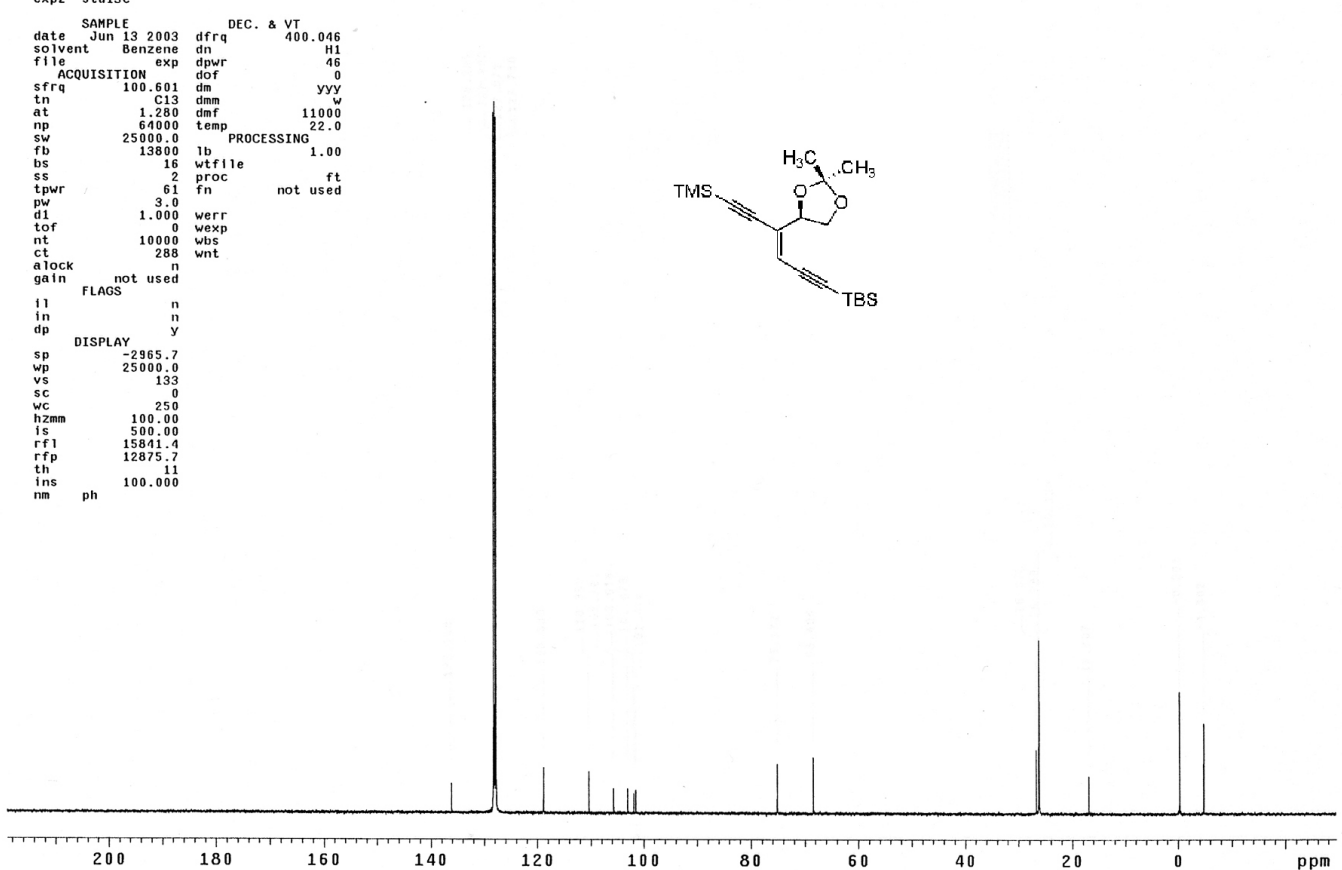

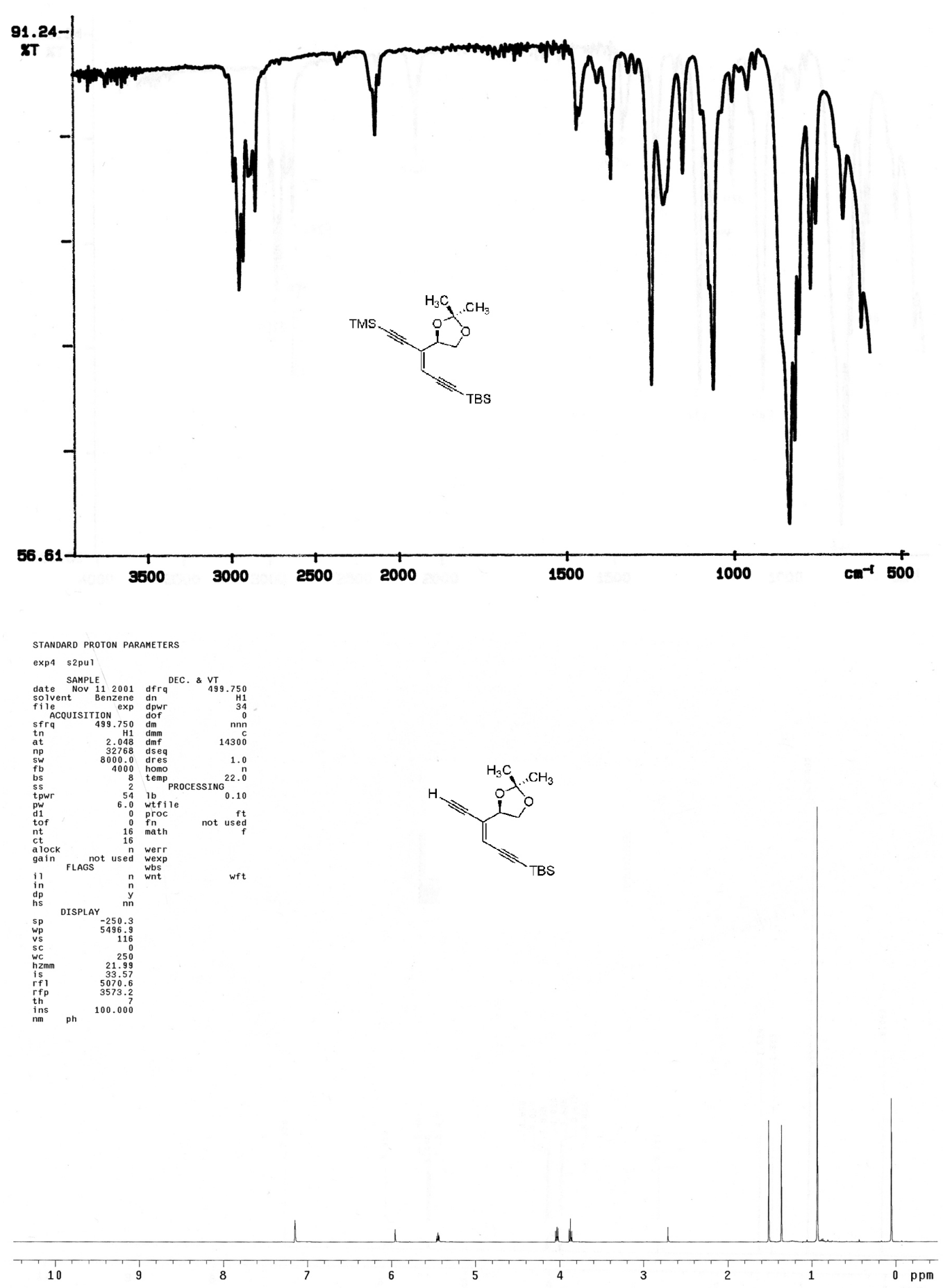

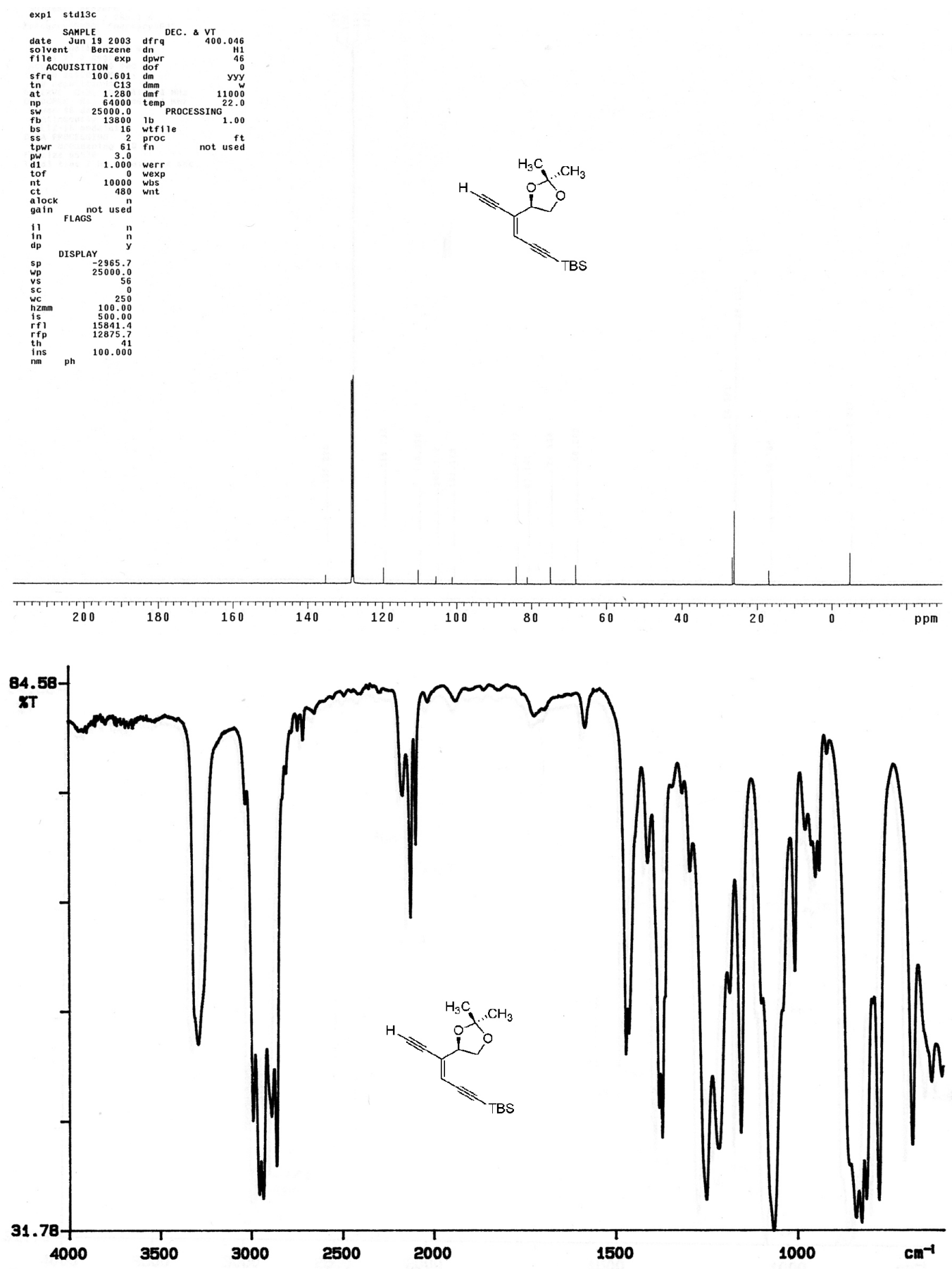

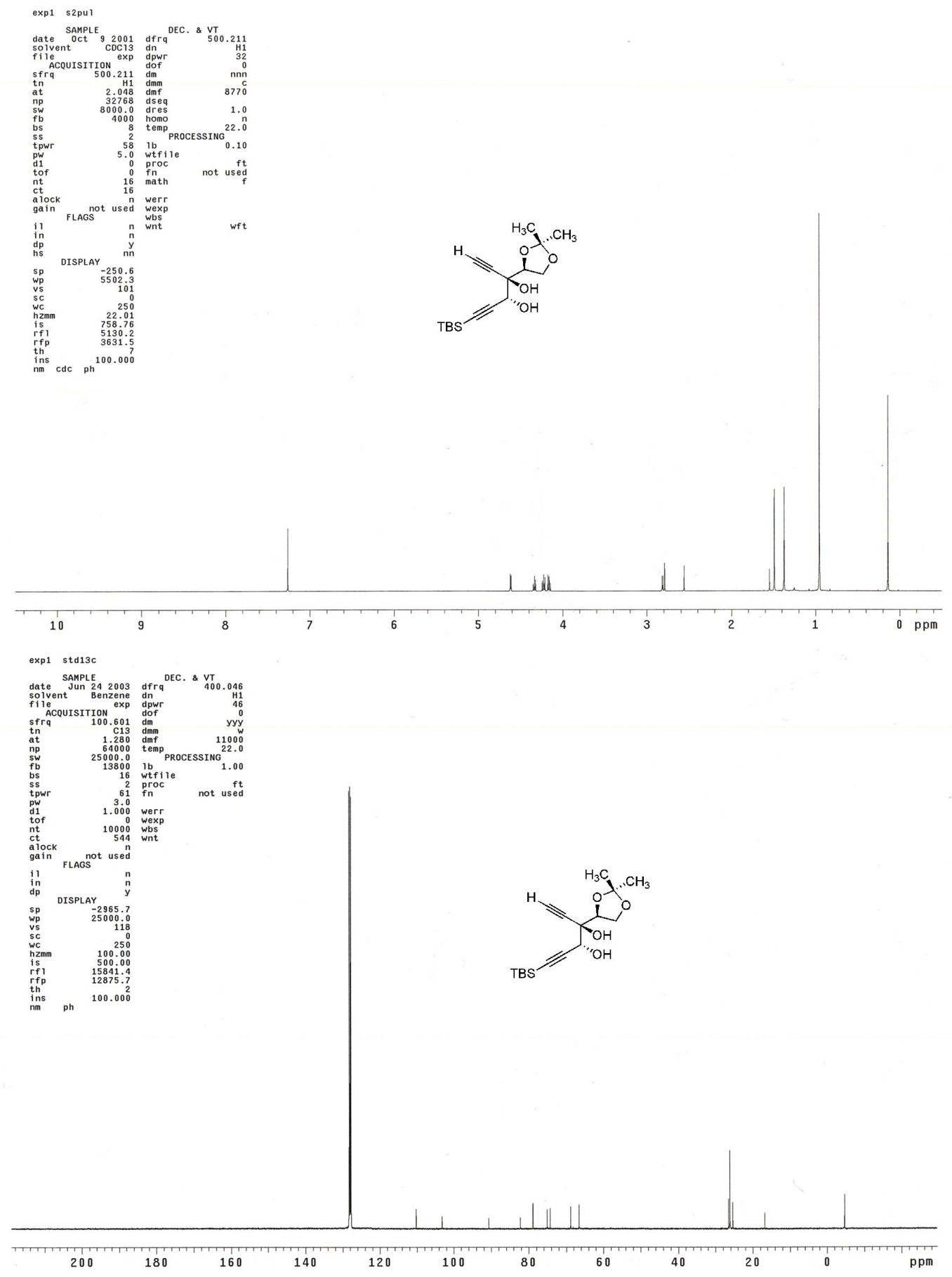

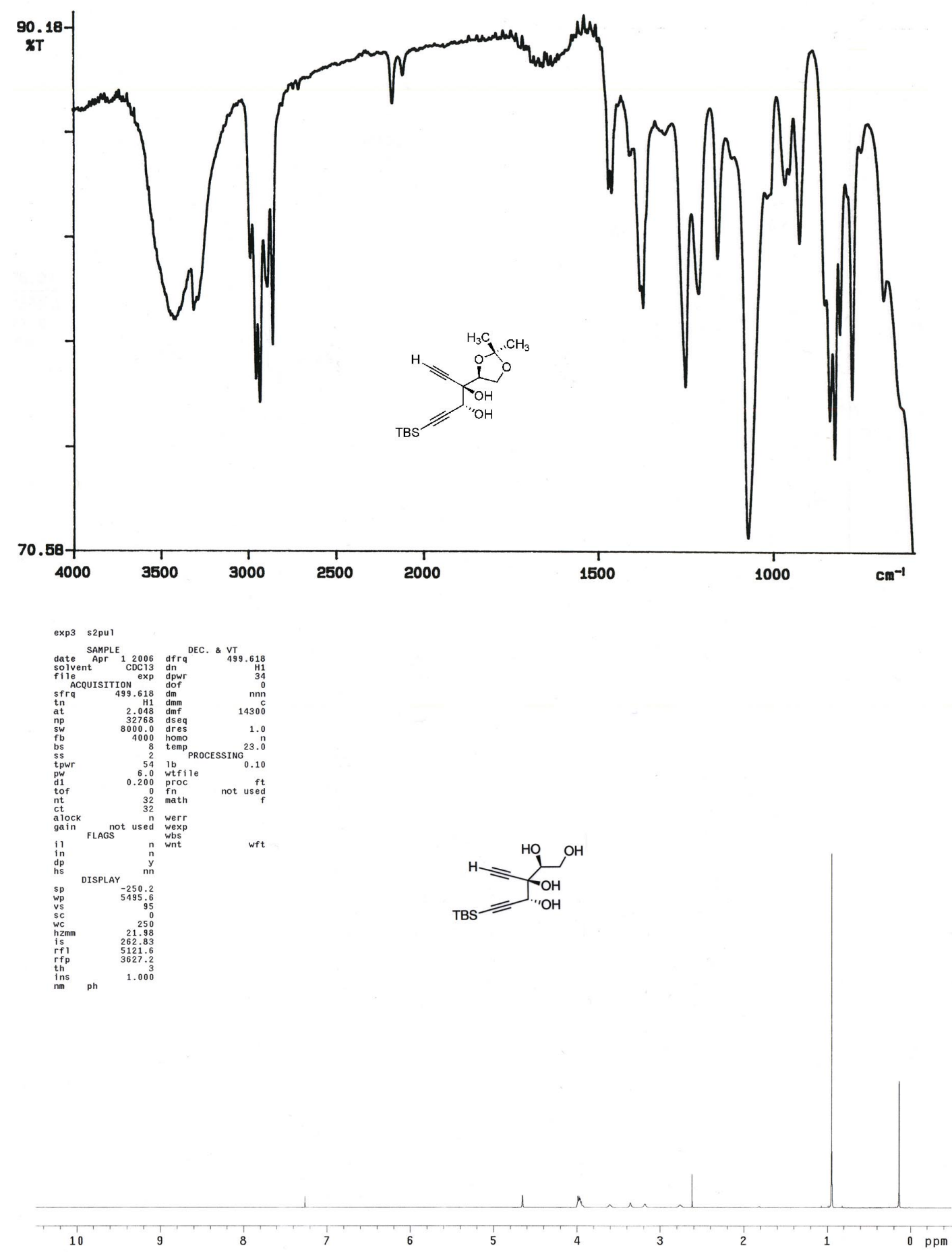

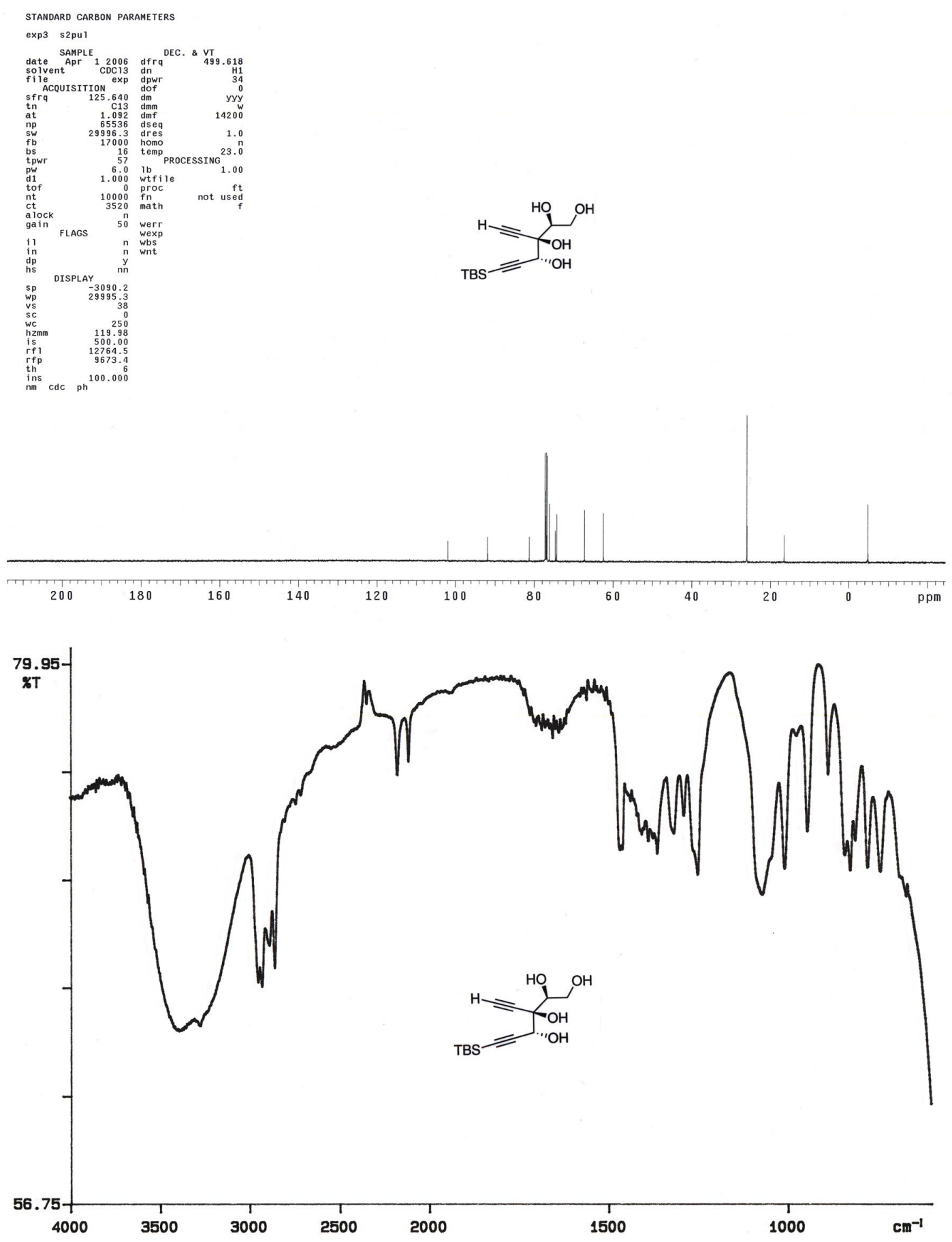

06/04/02 23: 35

$X: 16$ scans, $4.0 \mathrm{~cm}-1$ 

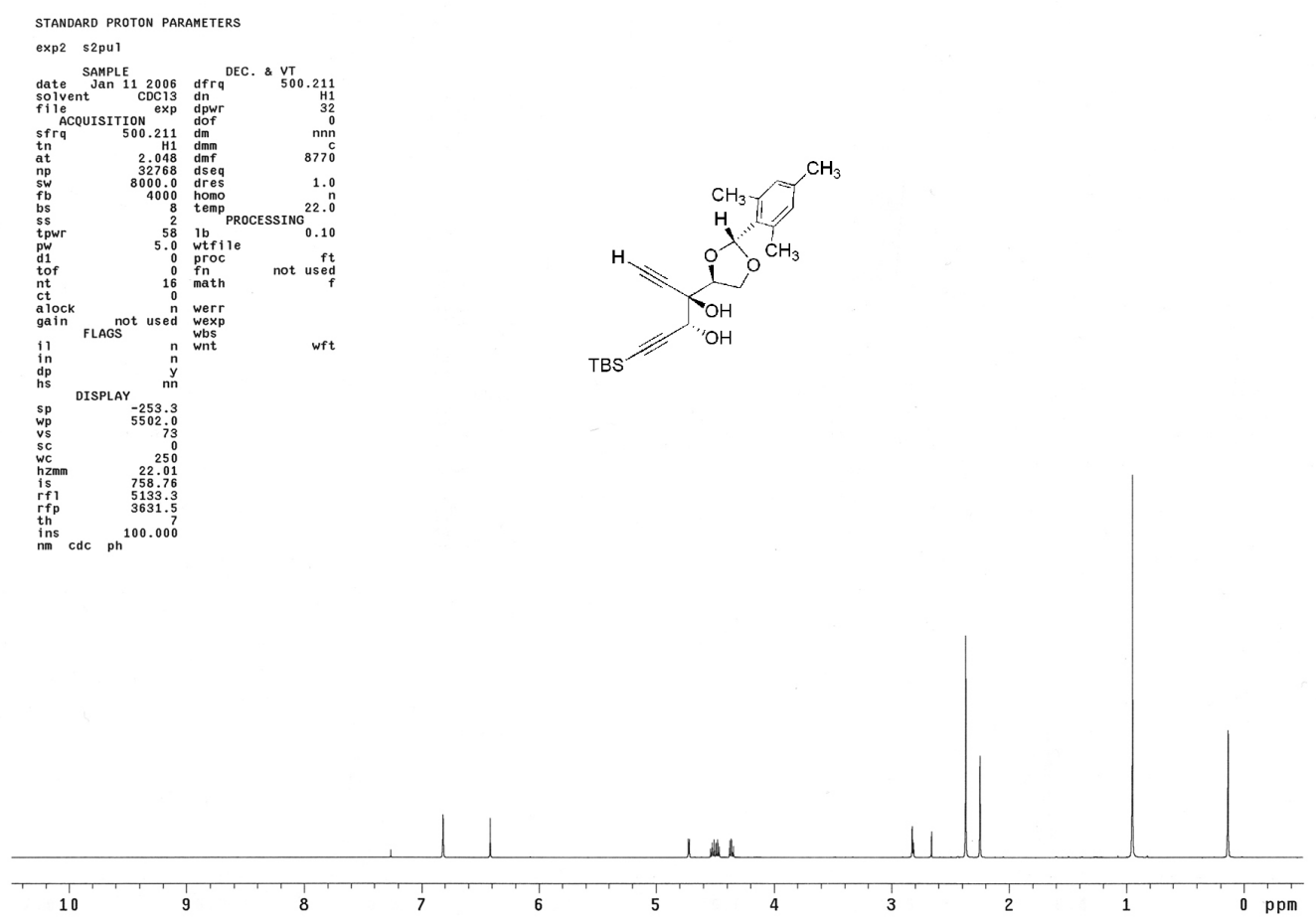

STANDARD CARBON PARAMETERS

exp2 s2pur
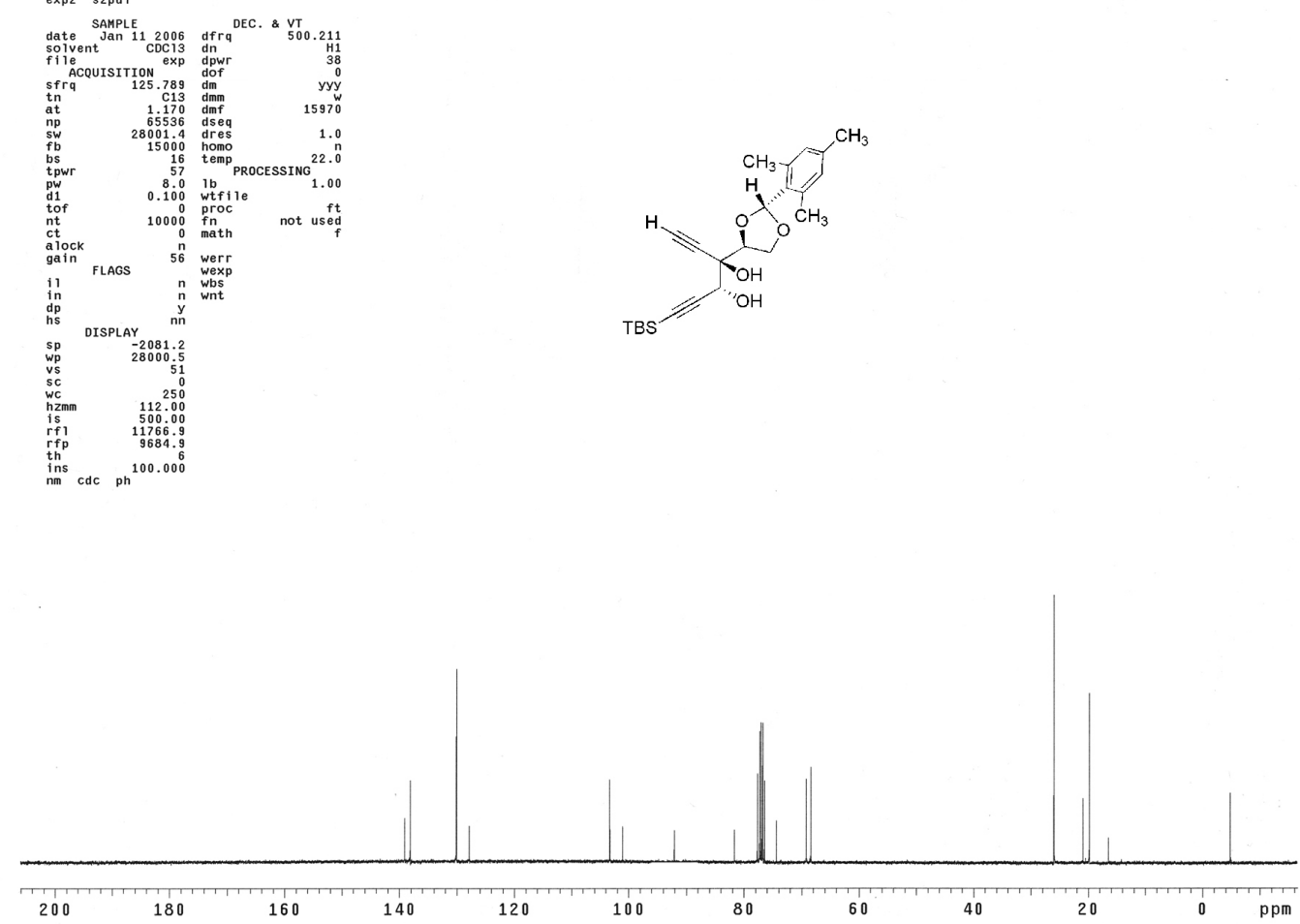
正目
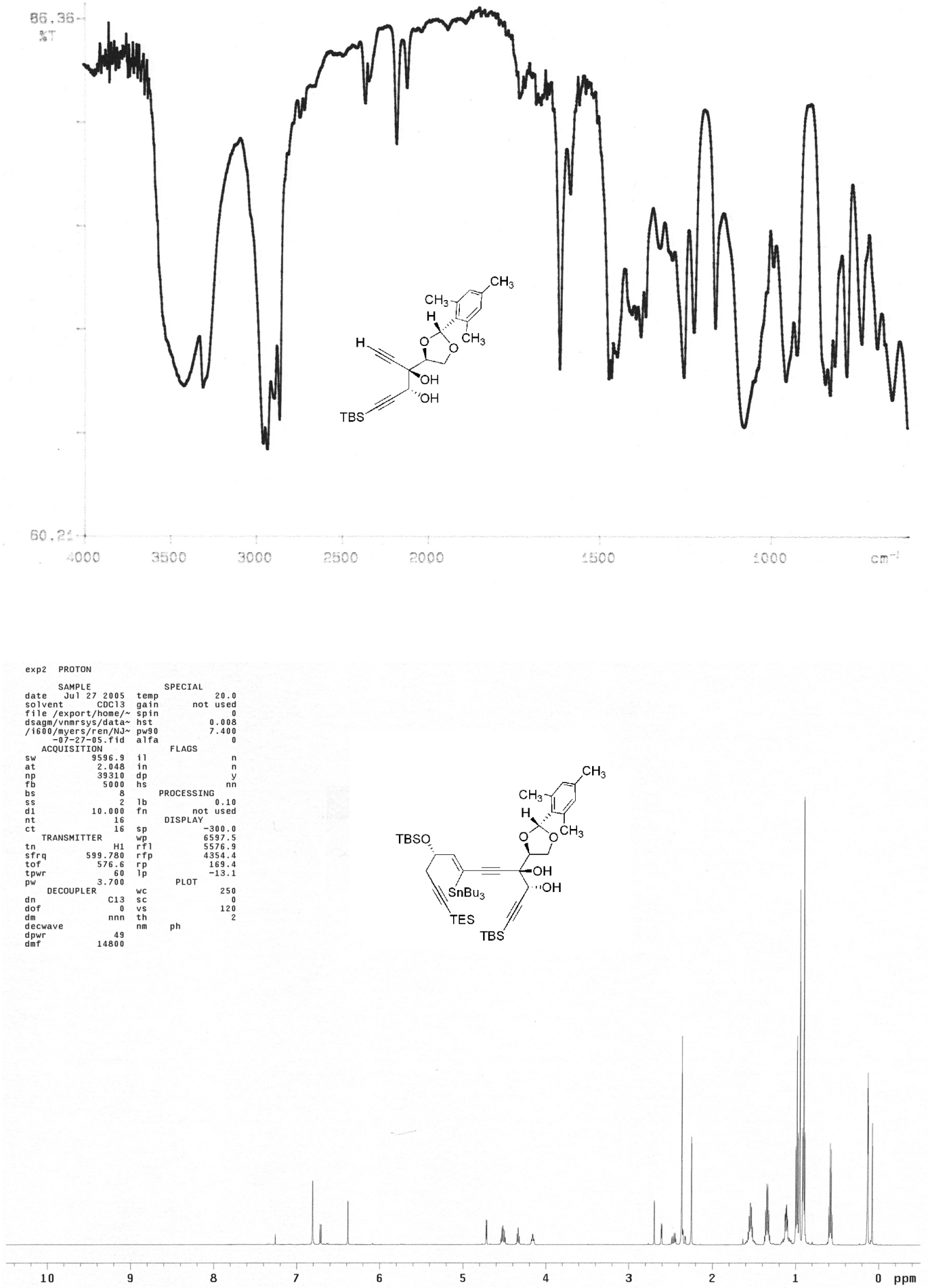

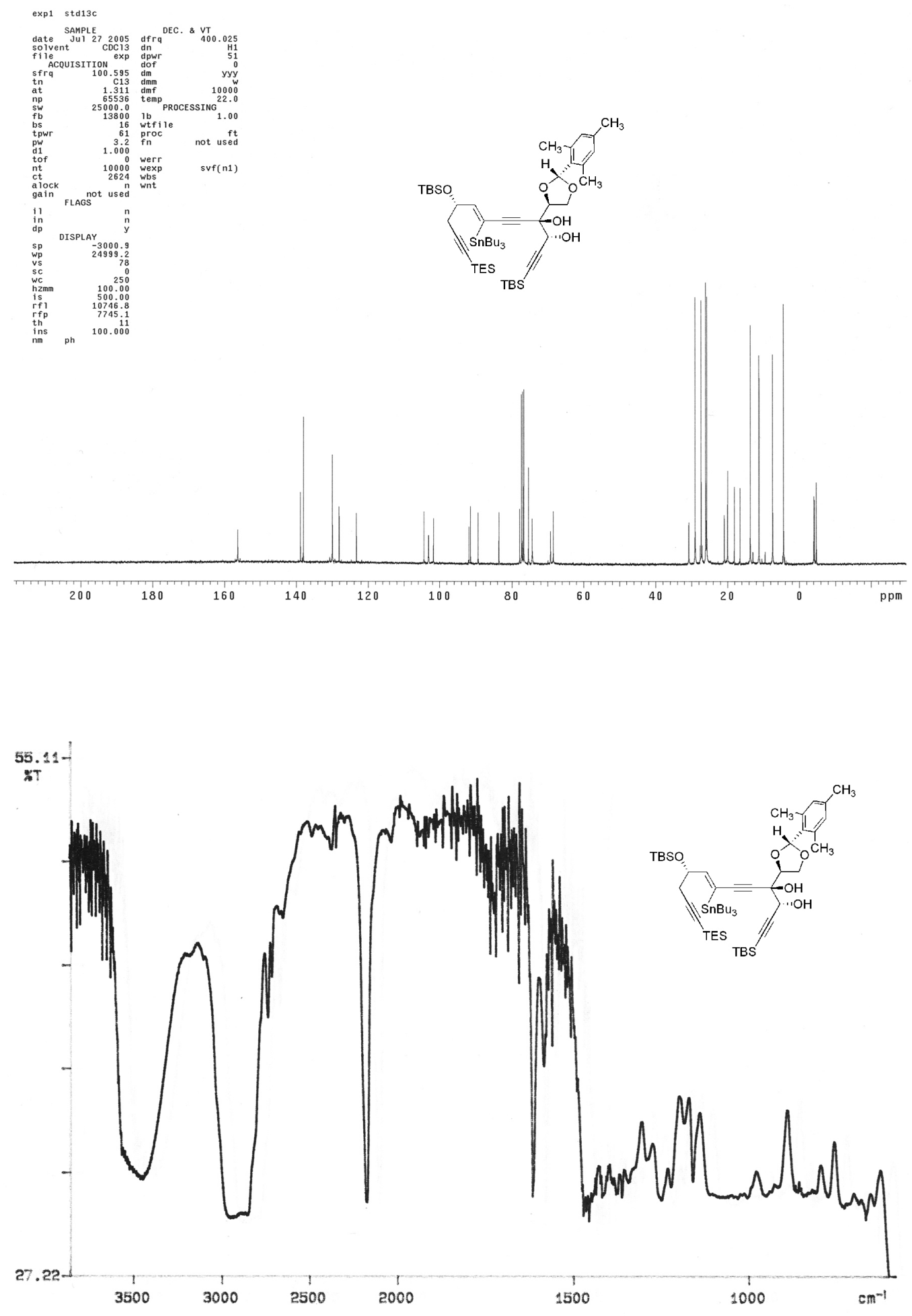

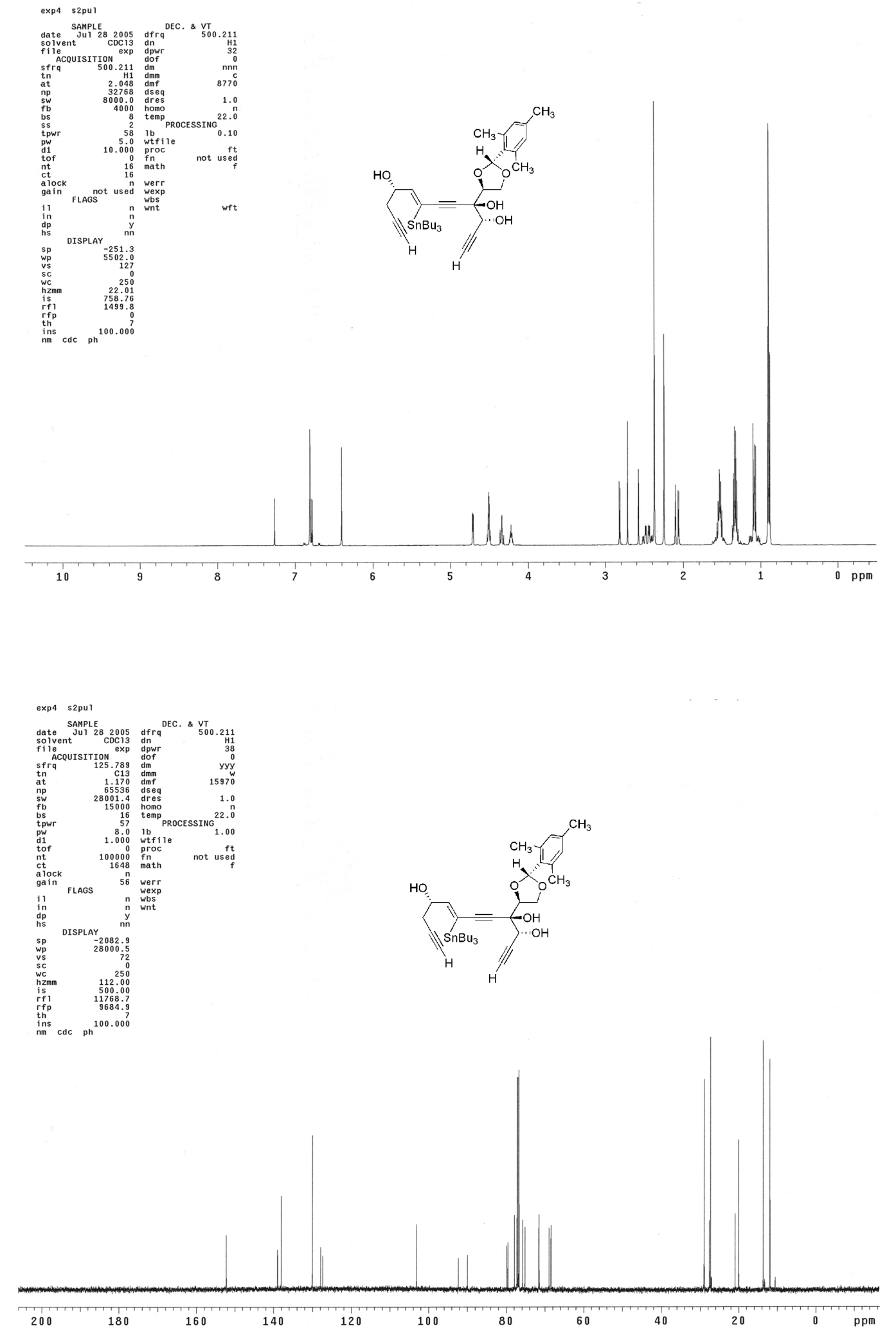

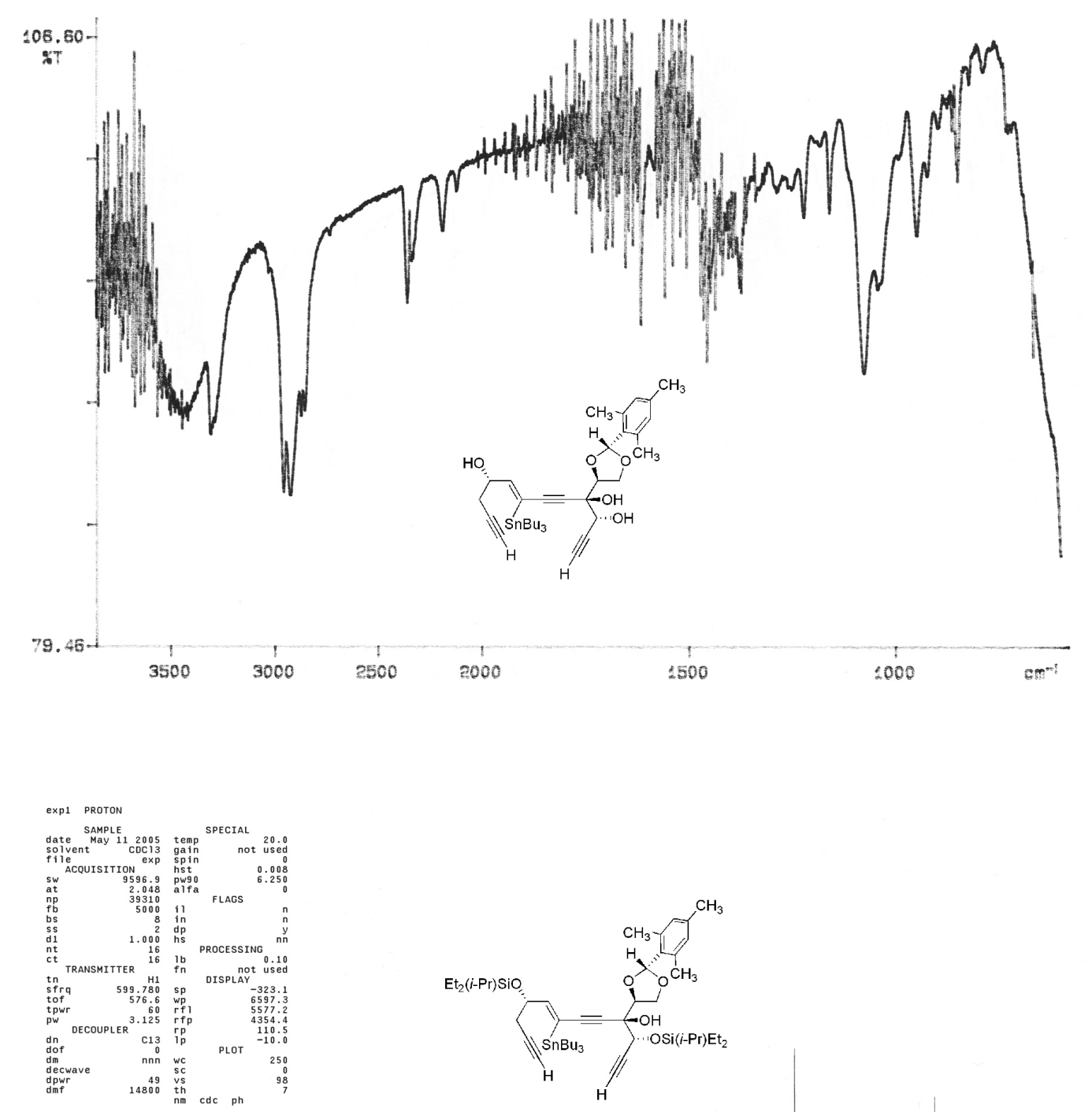

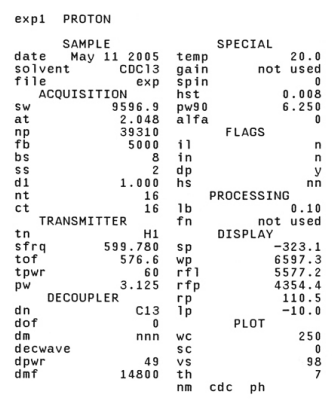



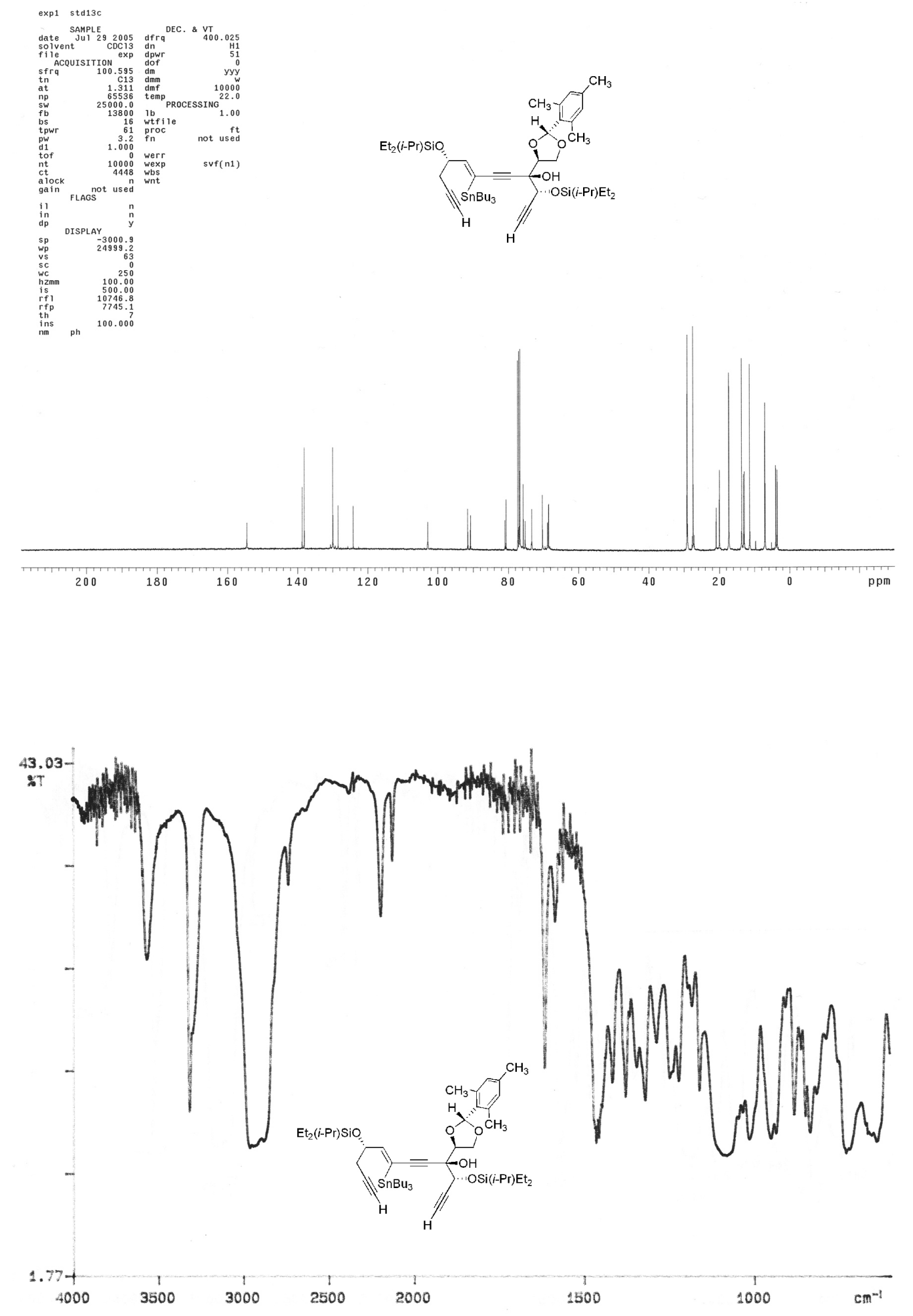

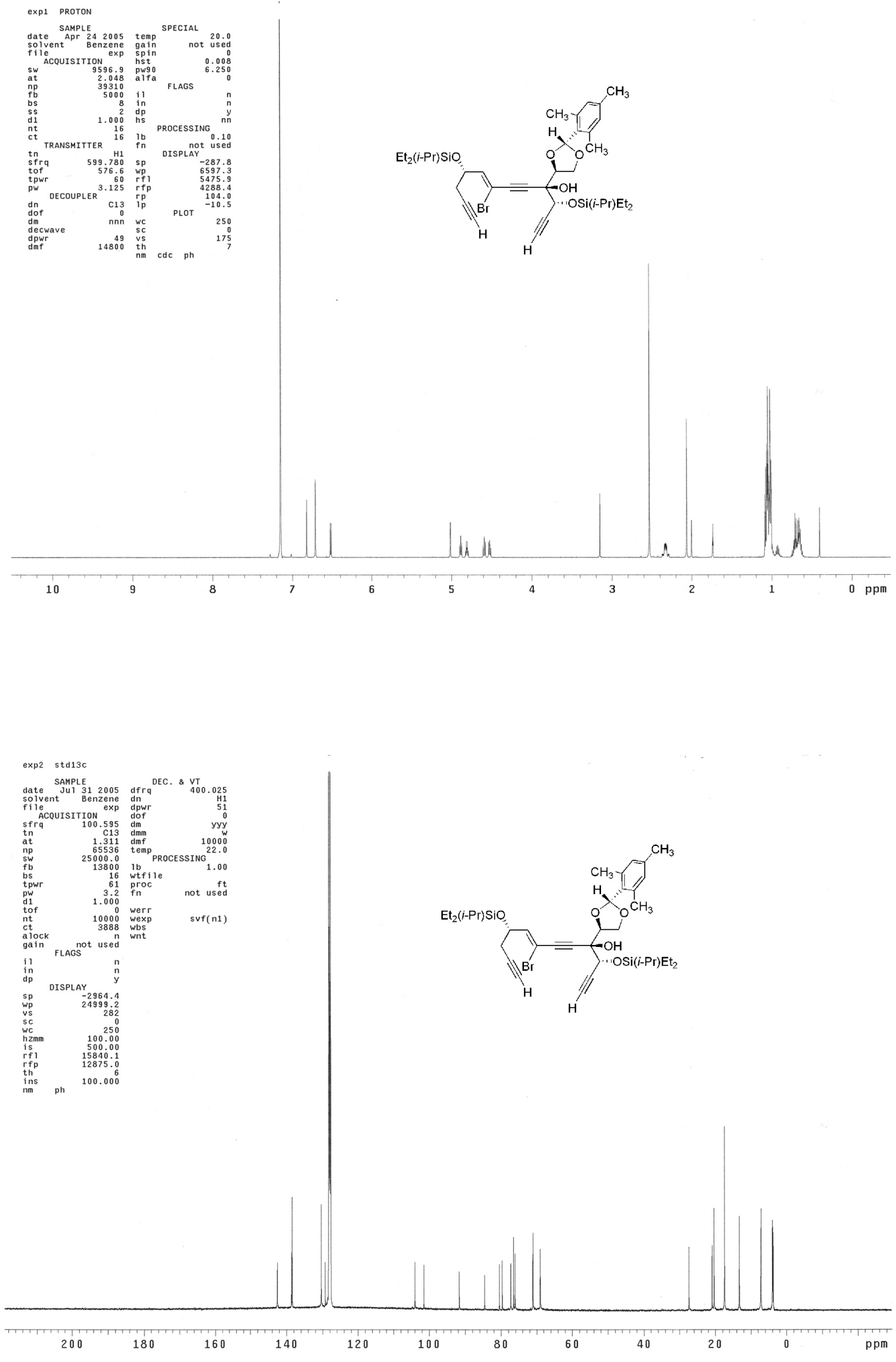


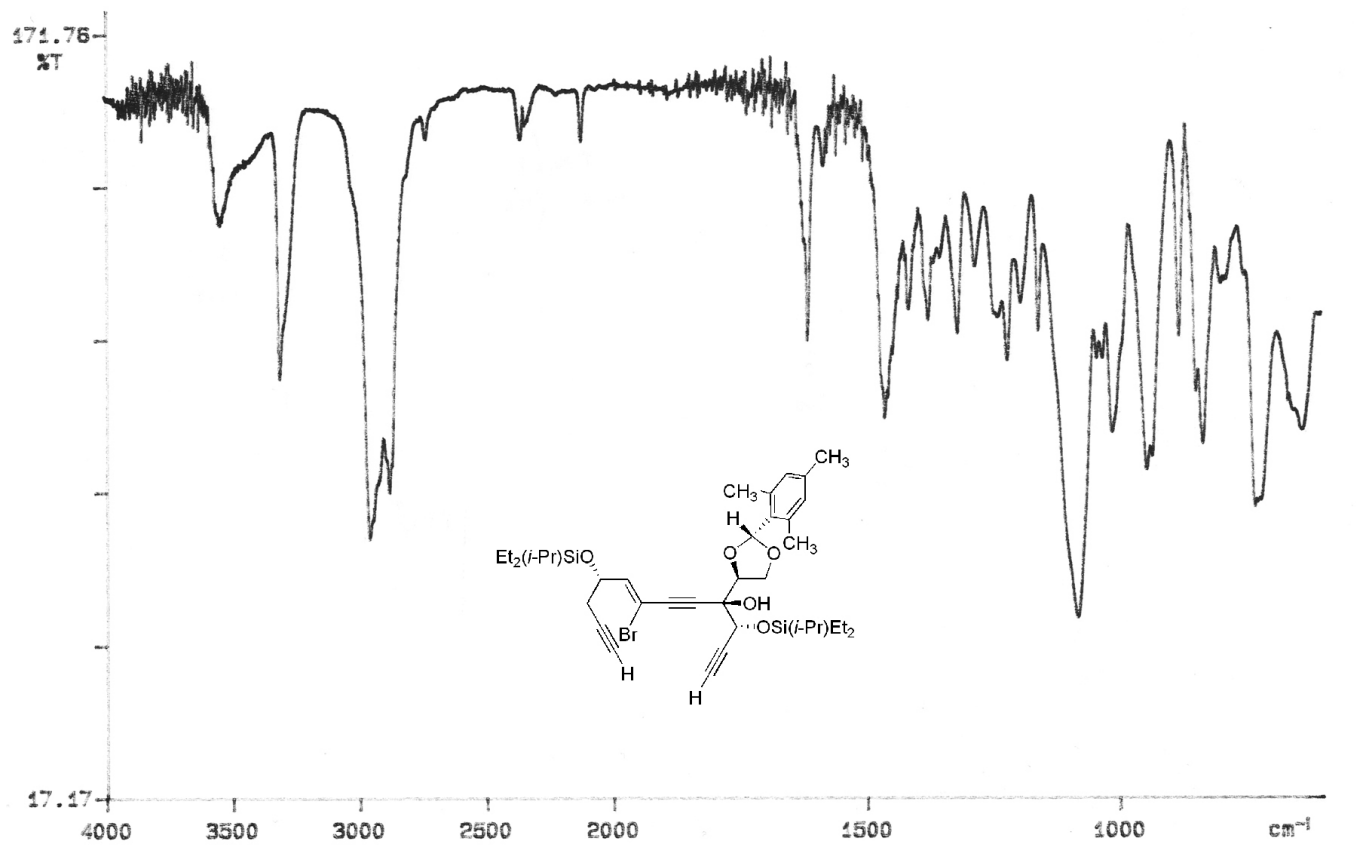

The ${ }^{1} \mathrm{H}$ NMR spectrum reproduced below was recorded from a partially-concentrated solution of the compound (structure shown) in $\mathrm{C}_{6} \mathrm{D}_{6}$.
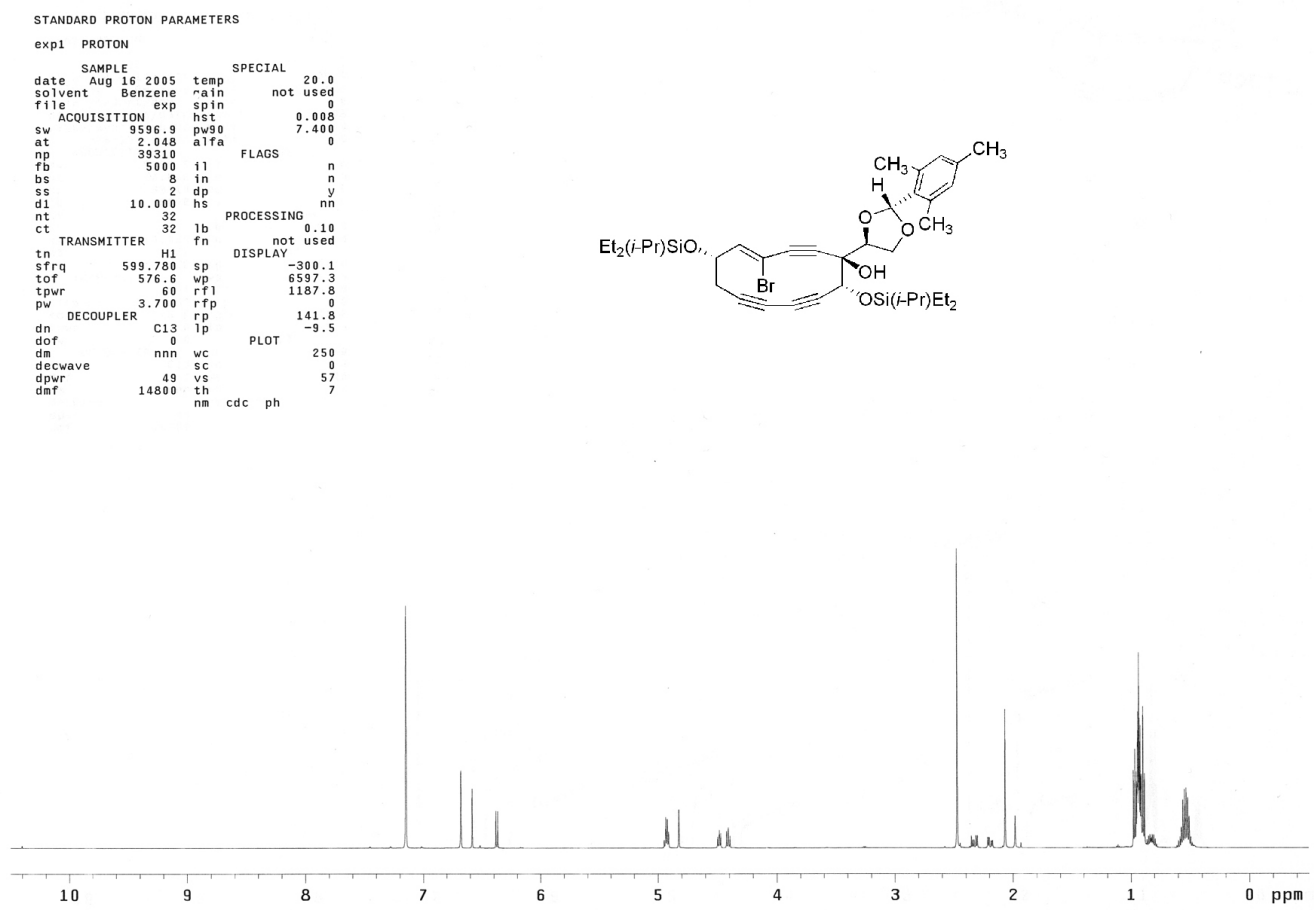
The ${ }^{13} \mathrm{C}$ NMR spectrum reproduced below was recorded from a partially-concentrated solution of the compound (structure shown) in $\mathrm{C}_{6} \mathrm{D}_{6}$.

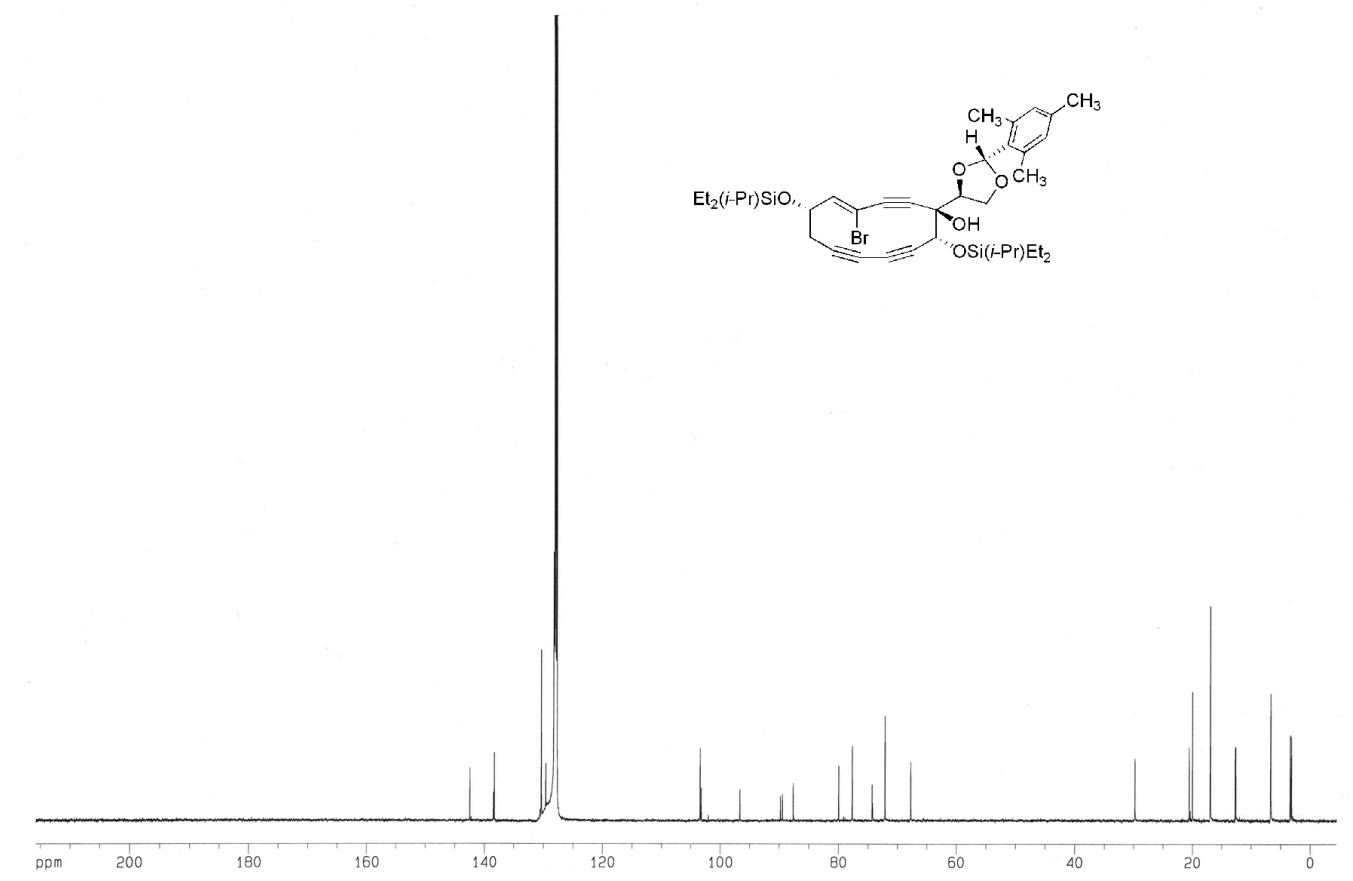

The infrared spectrum reproduced below was recorded from a partially-concentrated solution of the compound (structure shown) in $\mathrm{C}_{6} \mathrm{D}_{6}$ (pure $\mathrm{C}_{6} \mathrm{D}_{6}$ was used for the background spectrum).

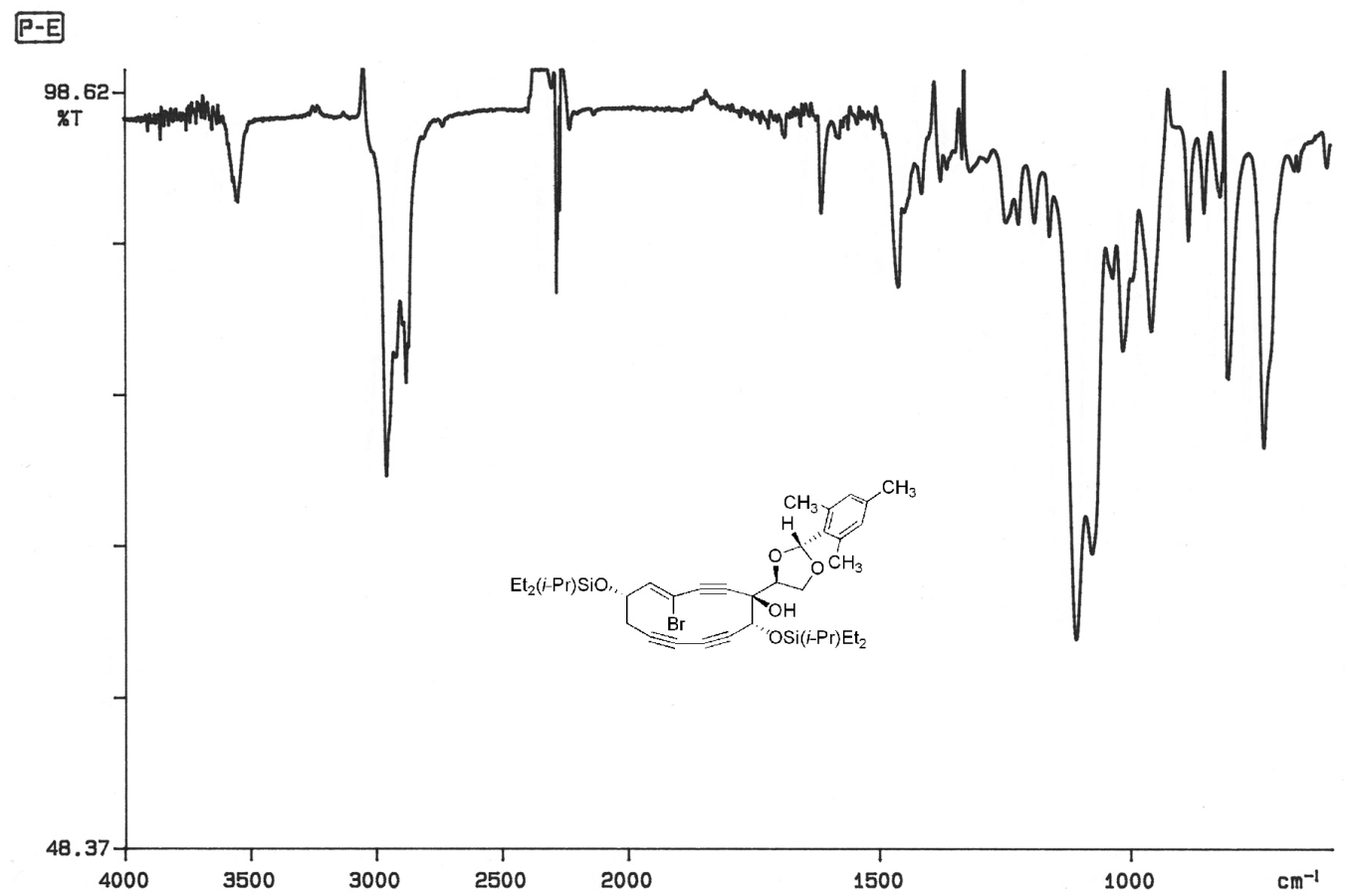


The ${ }^{1} \mathrm{H}$ NMR spectrum reproduced below was recorded from a partially-concentrated solution of the compound (structure shown) in $\mathrm{CD}_{3} \mathrm{CN}$.
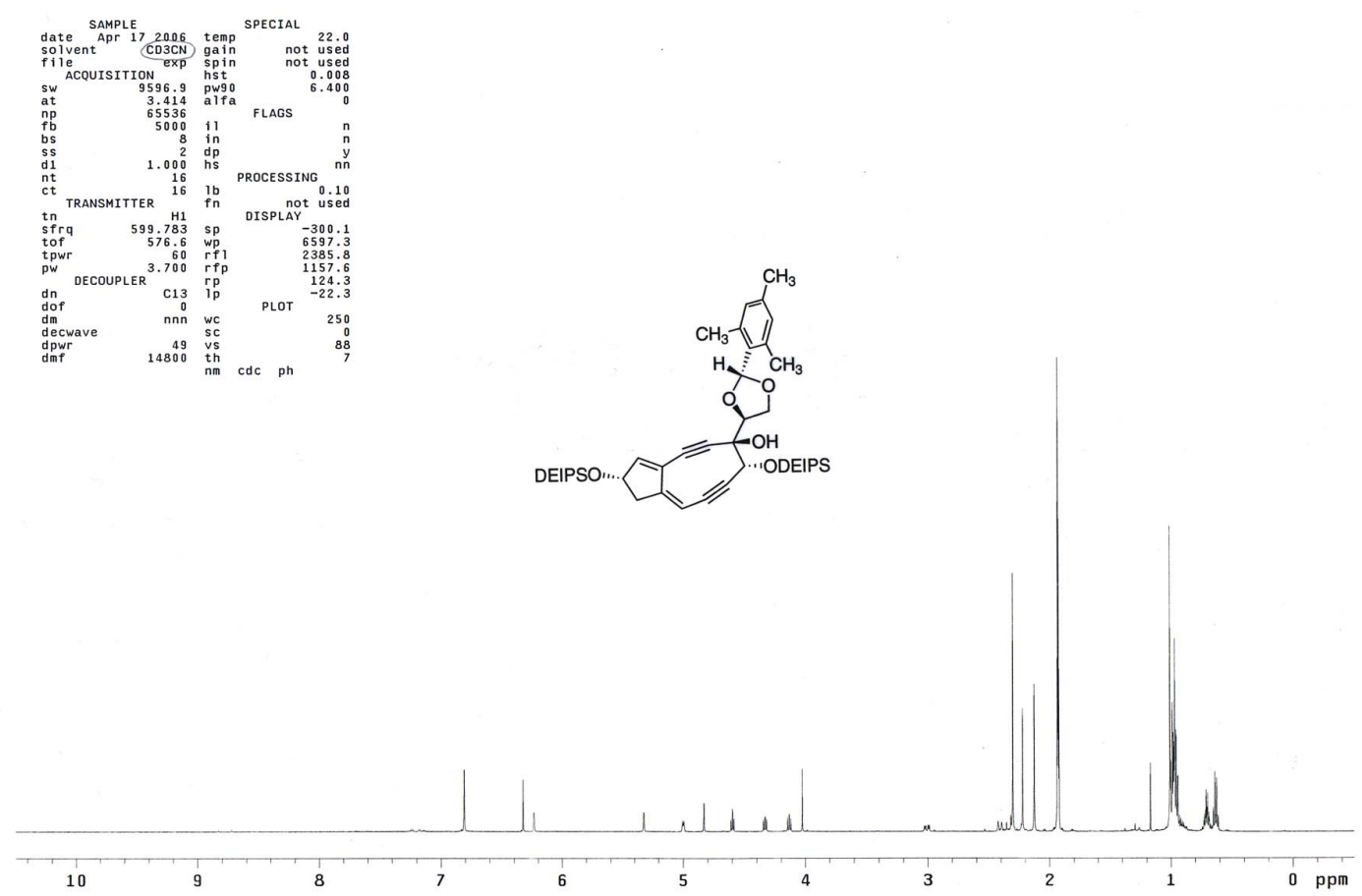

The ${ }^{13} \mathrm{C}$ NMR spectrum reproduced below was recorded from a partially-concentrated solution of the compound (structure shown) in $\mathrm{C}_{6} \mathrm{D}_{6}$.
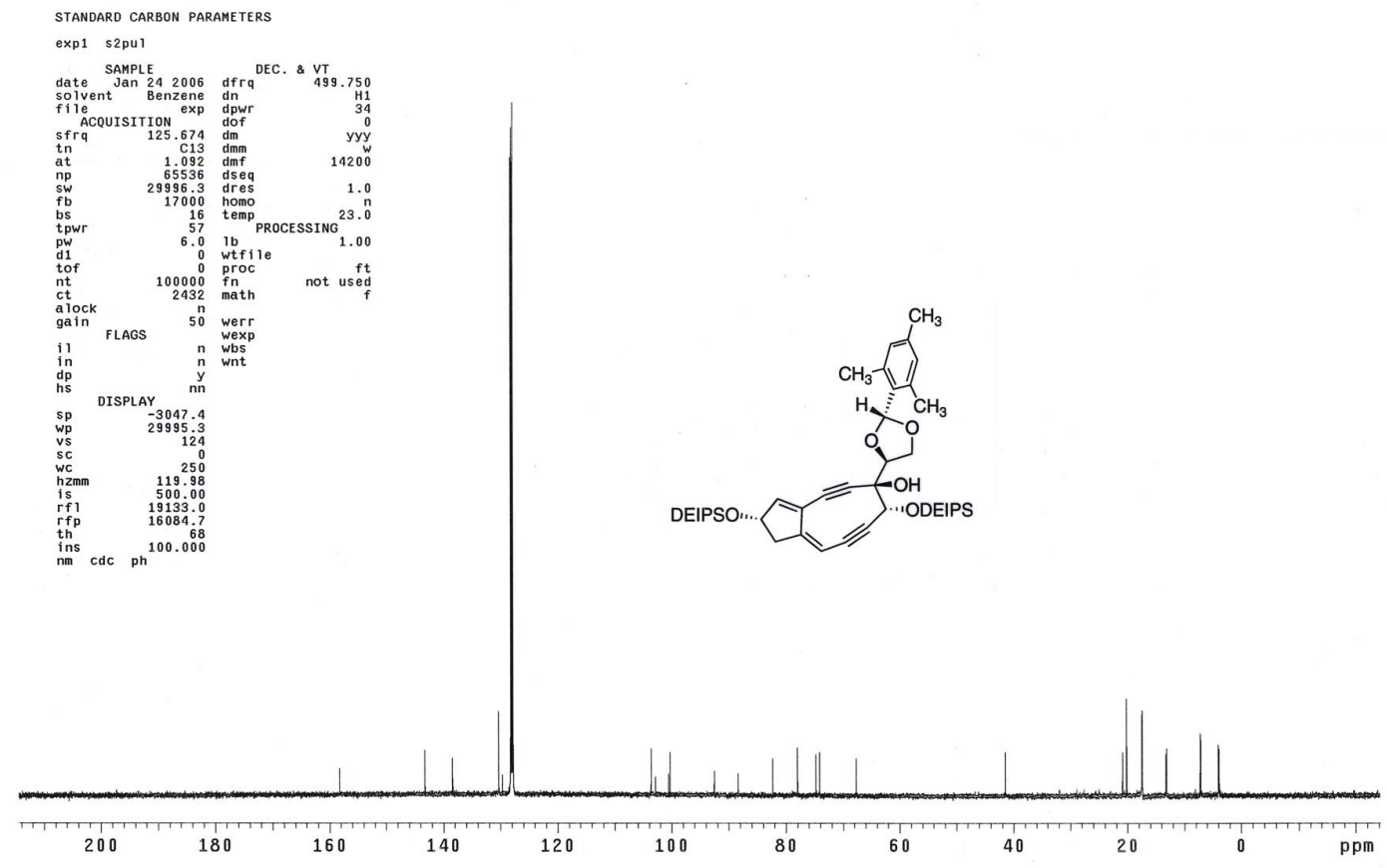
The infrared spectrum reproduced below was recorded from a partially-concentrated solution of the compound (structure shown) in $\mathrm{CD}_{3} \mathrm{CN}$ (pure $\mathrm{CD}_{3} \mathrm{CN}$ was used for background spectrum).

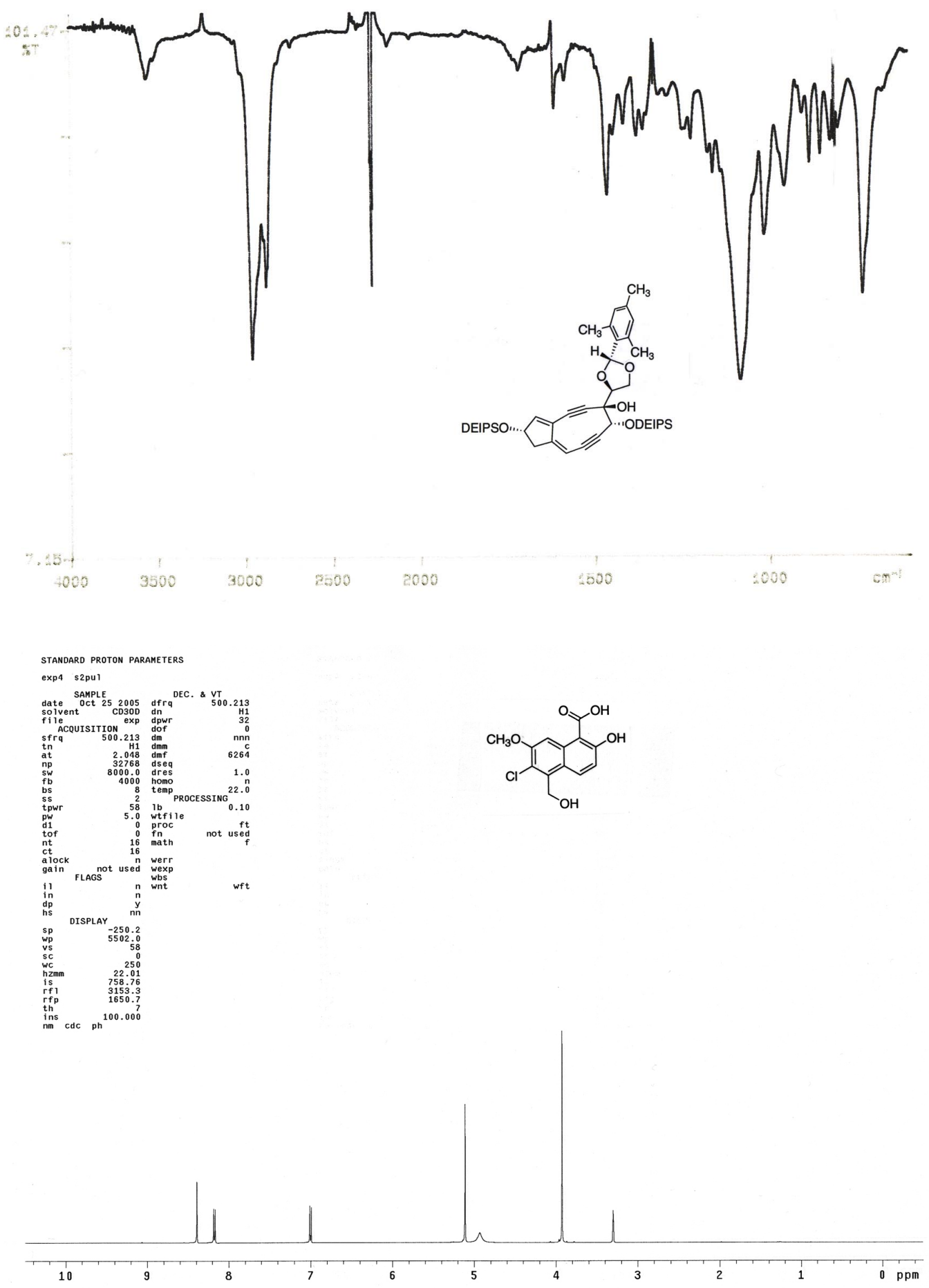



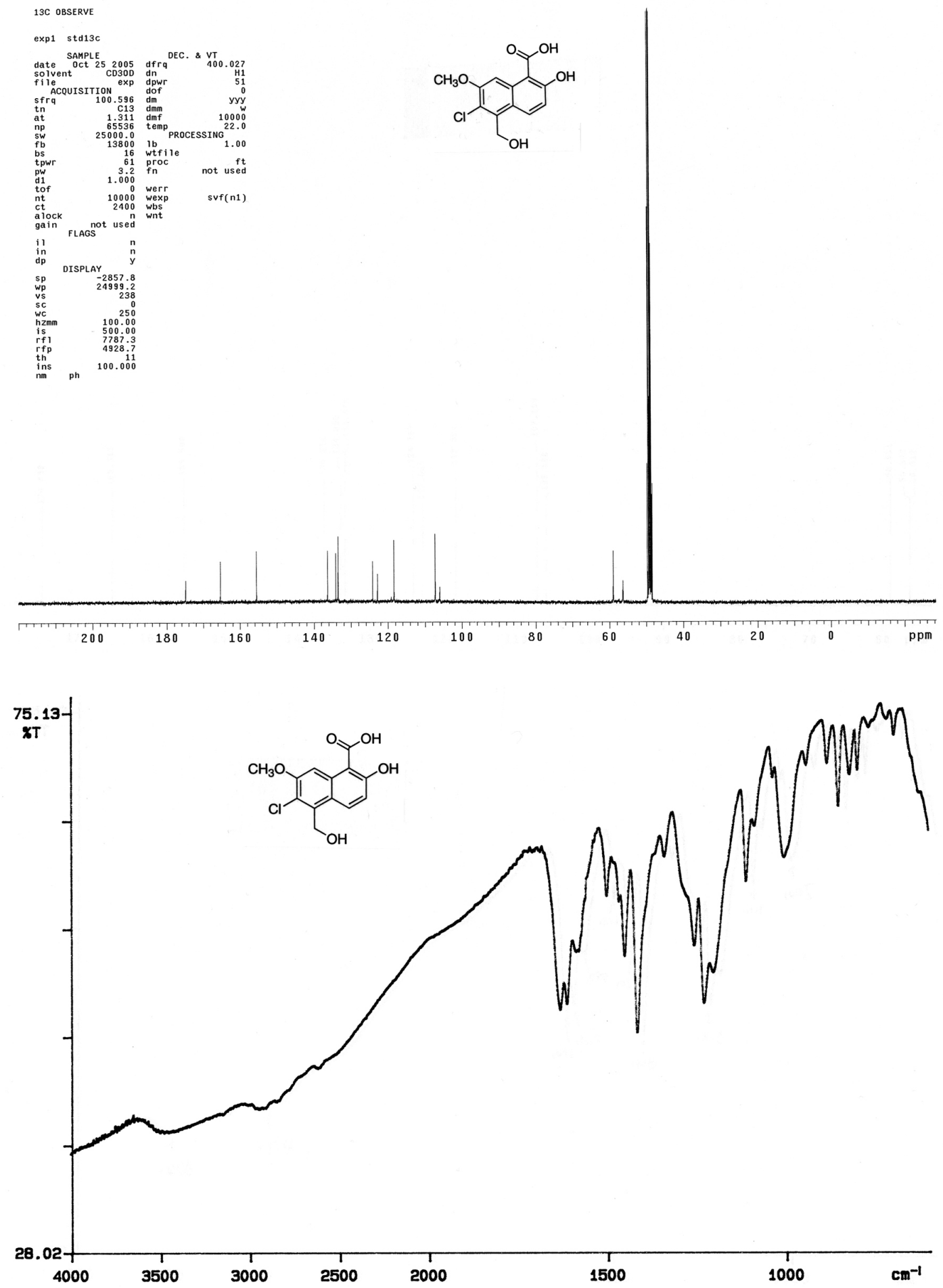

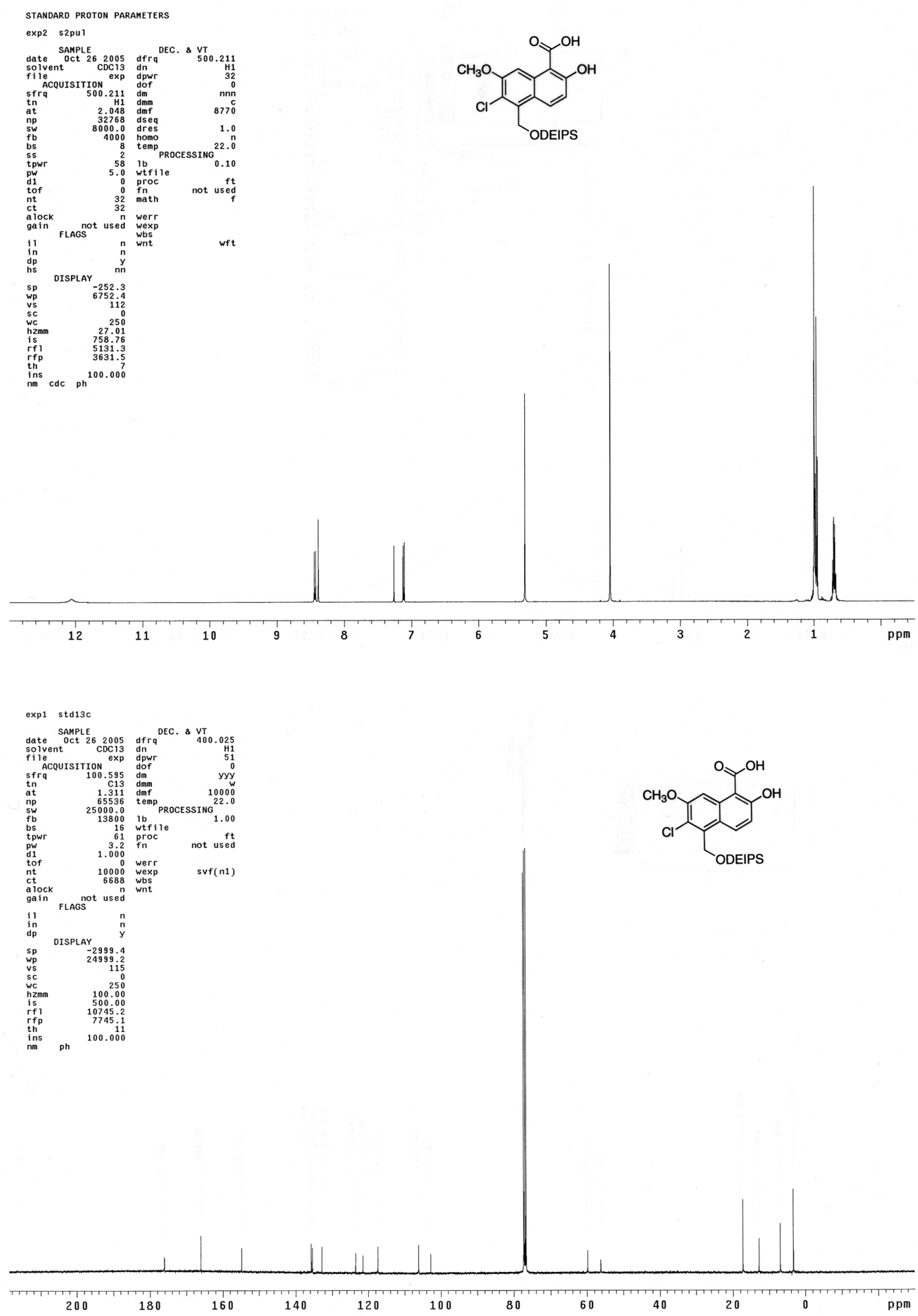


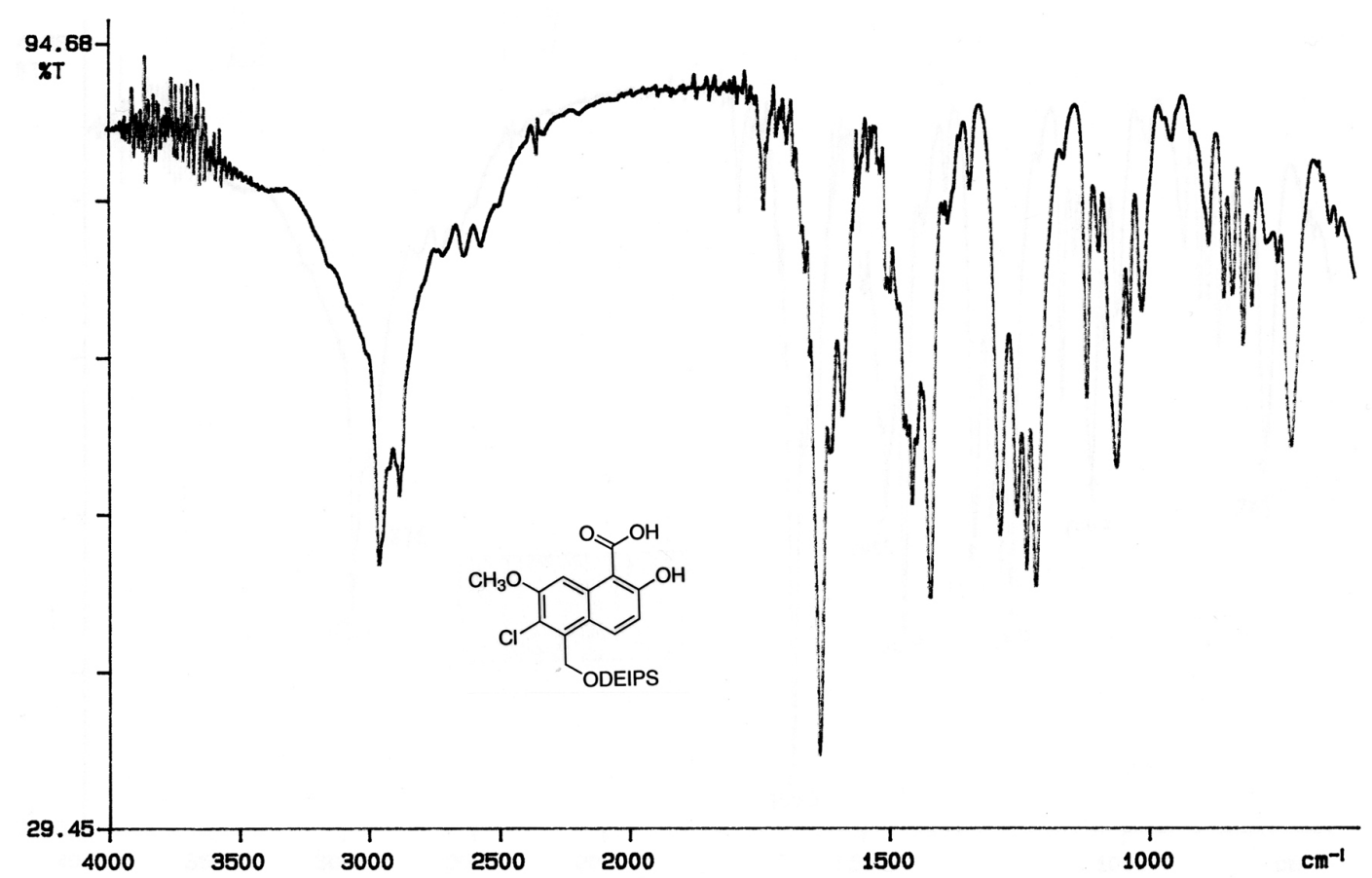

The ${ }^{1} \mathrm{H}$ NMR spectrum reproduced below was recorded from a partially-concentrated solution of the compound (structure shown) in $\mathrm{C}_{6} \mathrm{D}_{6}$.
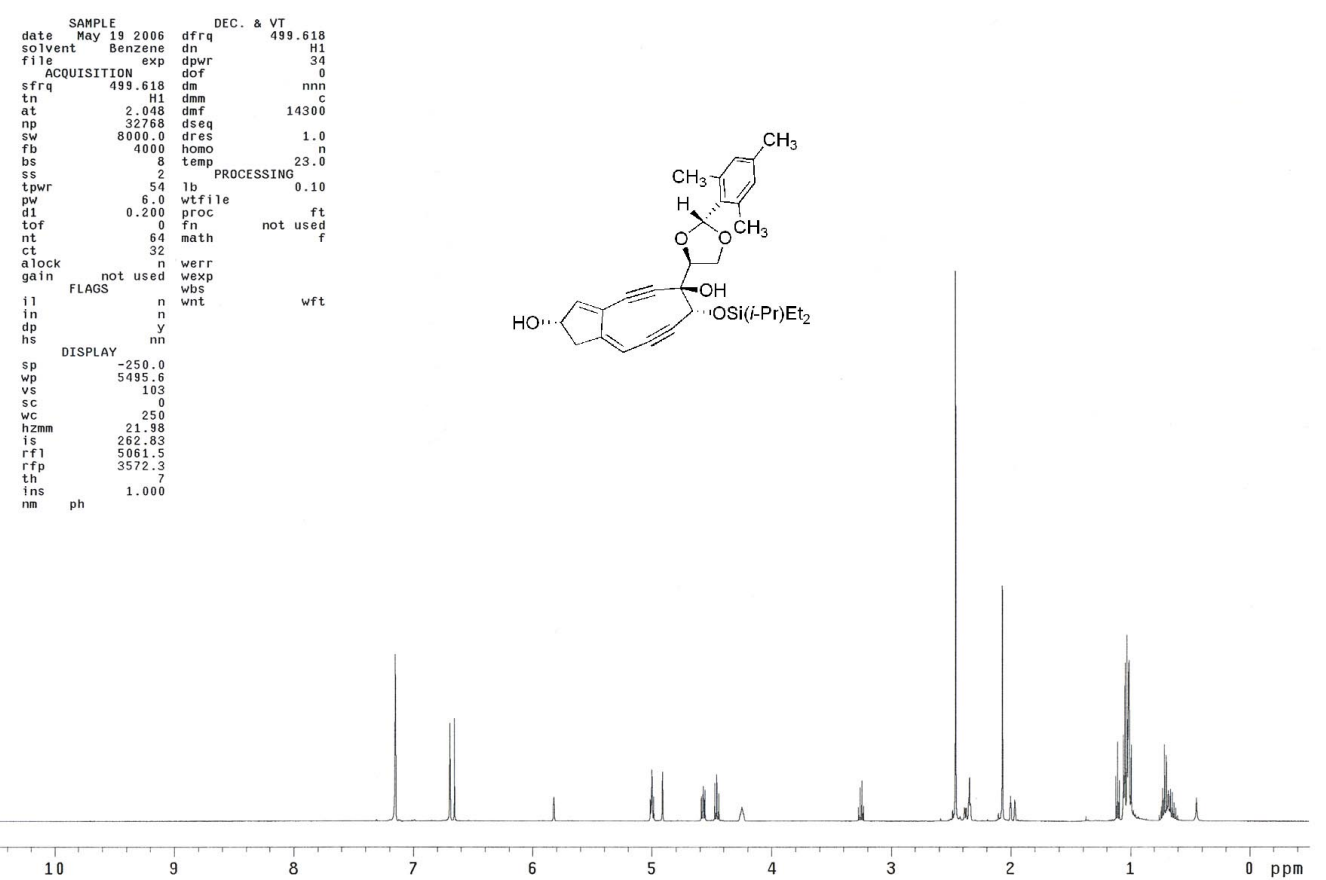

The ${ }^{1} \mathrm{H}$ NMR spectrum reproduced below was recorded from a partially-concentrated solution of 
the compound (structure shown) in $\mathrm{C}_{6} \mathrm{D}_{6}$.
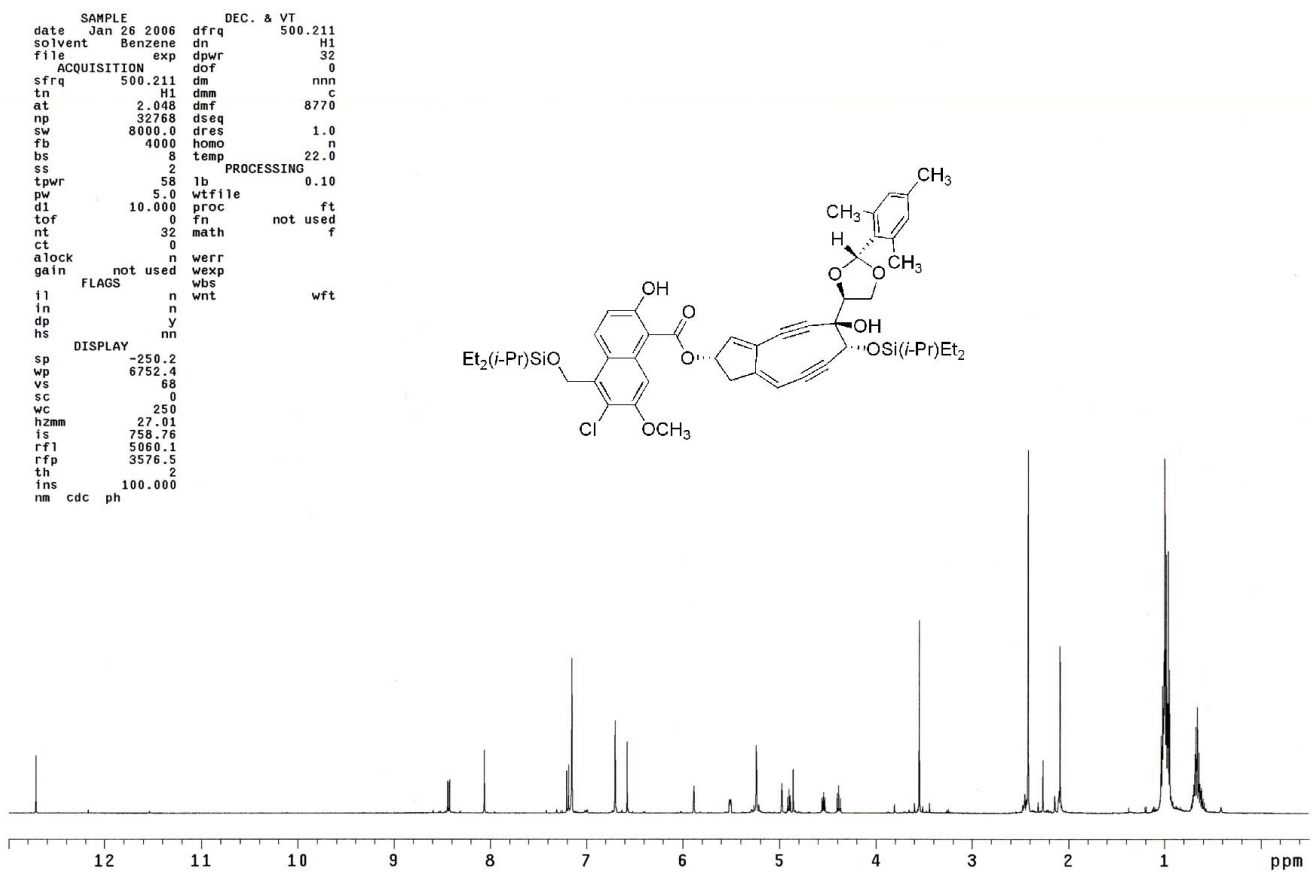

The ${ }^{13} \mathrm{C}$ NMR spectrum reproduced below was recorded from a partially-concentrated solution of the compound (structure shown) in $\mathrm{C}_{6} \mathrm{D}_{6}$.
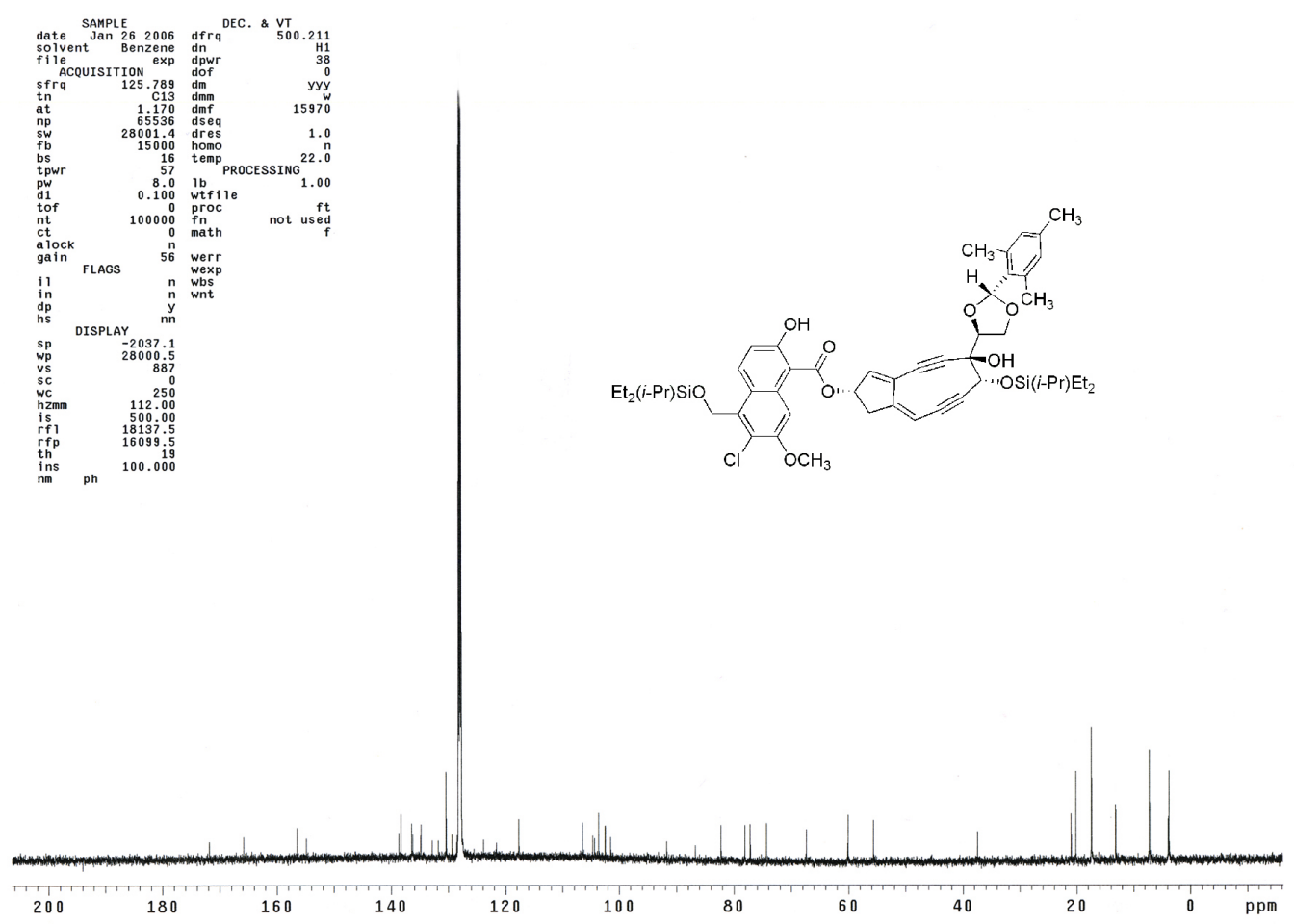
The ${ }^{1} \mathrm{H}$ NMR spectrum reproduced below was recorded from a partially-concentrated solution of the compound (structure shown) in $\mathrm{C}_{6} \mathrm{D}_{6}$.

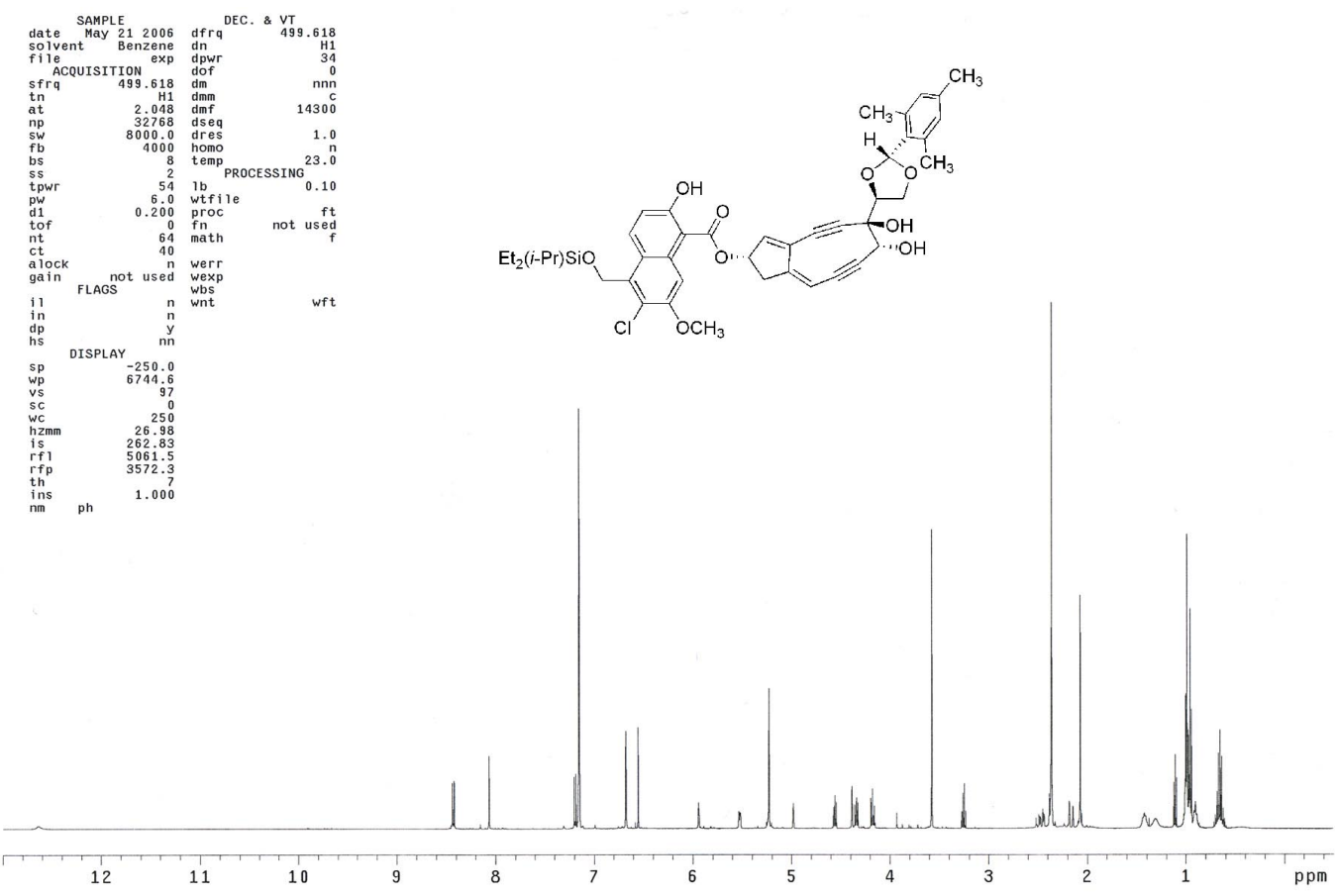

The ${ }^{1} \mathrm{H}$ NMR spectrum reproduced below was recorded from a partially-concentrated solution of the compound (structure shown) in $\mathrm{C}_{6} \mathrm{D}_{6}$.

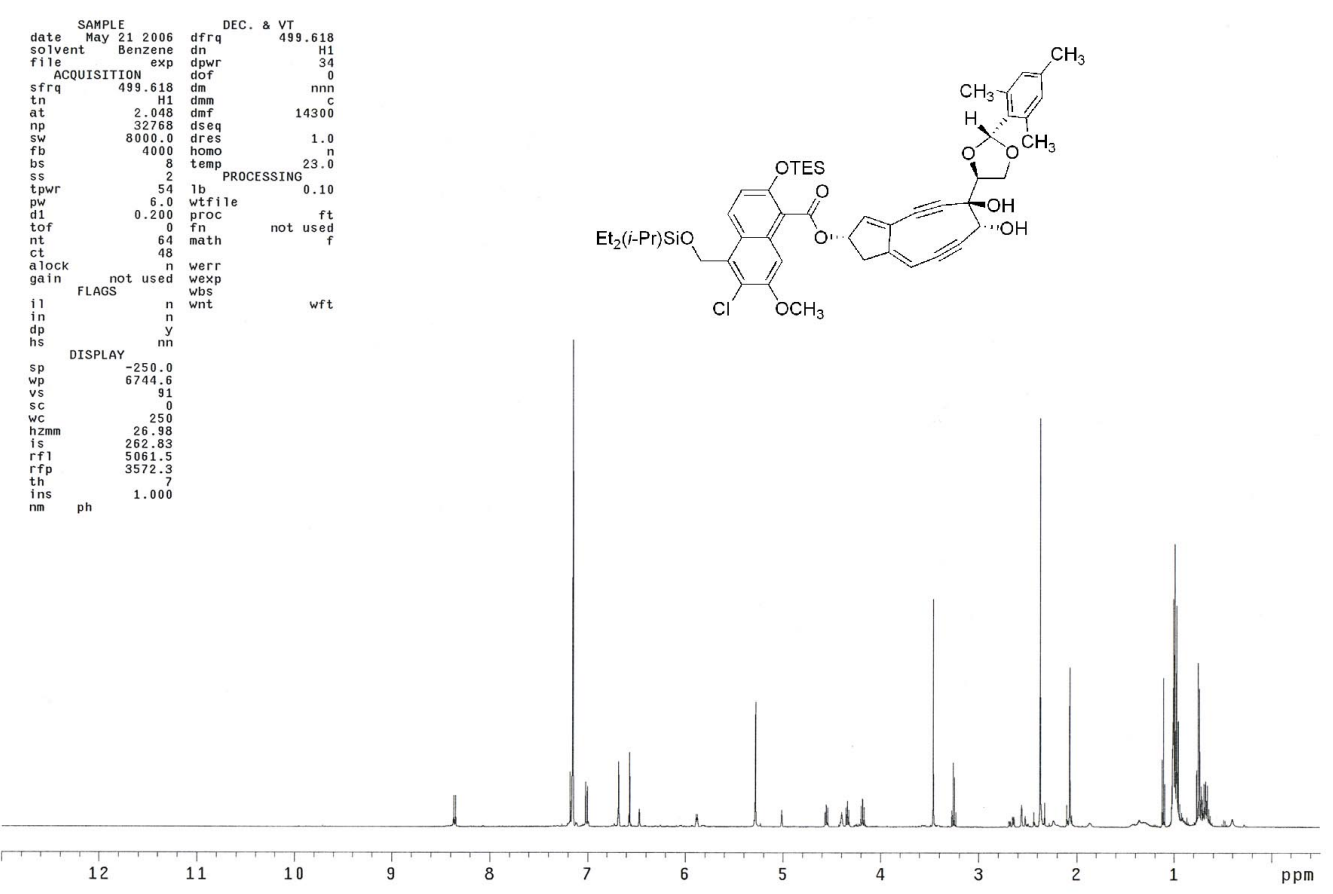


The ${ }^{1} \mathrm{H}$ NMR spectrum reproduced below was recorded from a partially-concentrated solution of the compound (structure shown) in $\mathrm{CD}_{3} \mathrm{CN}$.

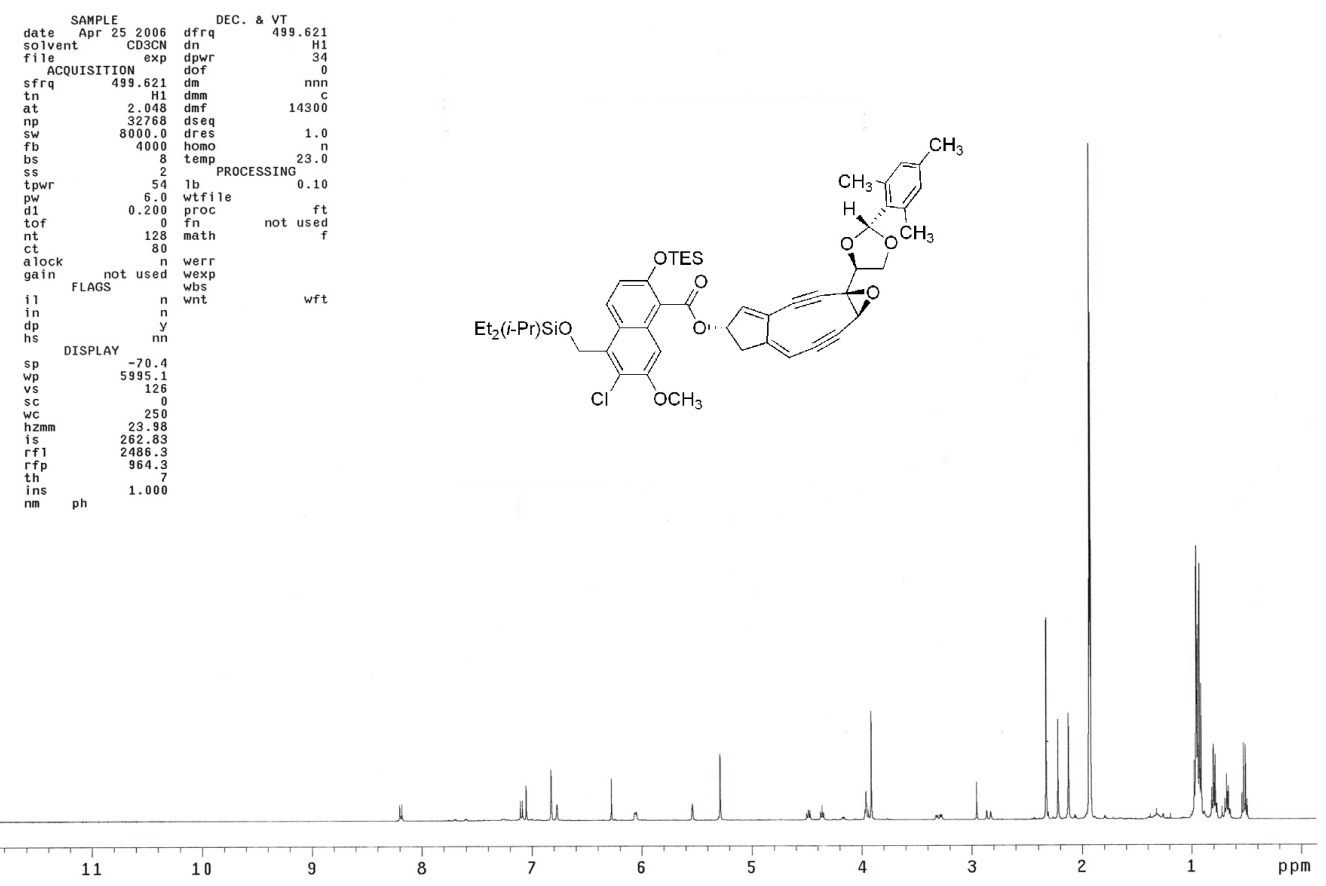

The ${ }^{1} \mathrm{H}$ NMR spectrum reproduced below was recorded from a partially-concentrated solution of the compound (structure shown) in a 1:1 mixture of DMSO- $\mathrm{d}_{6}$ and $\mathrm{CD}_{3} \mathrm{CN}$.

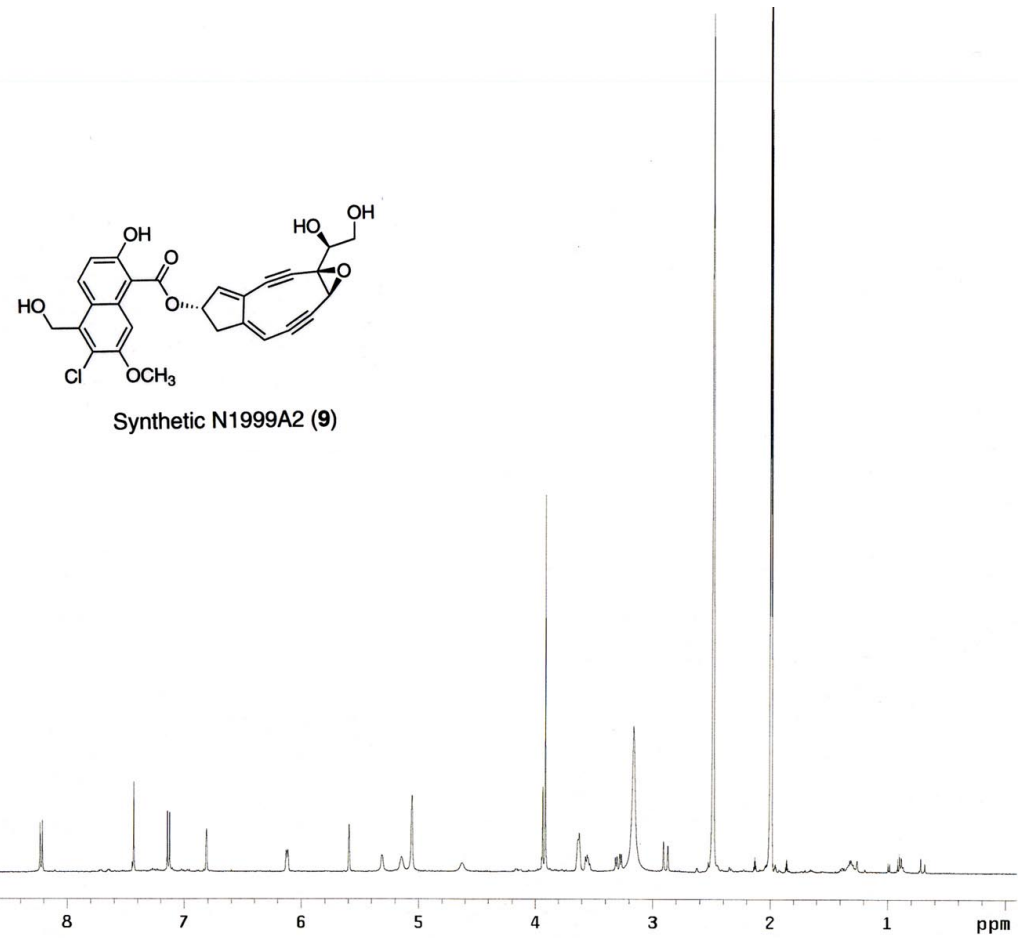

The ${ }^{1} \mathrm{H}$ NMR spectrum below was reproduced from Supporting Information, The First Total 
Synthesis of N1999A2: Absolute Stereochemistry and Stereochemical Implications into DNA Cleavage, J. Am. Chem. Soc. 2001, 123, 11294.

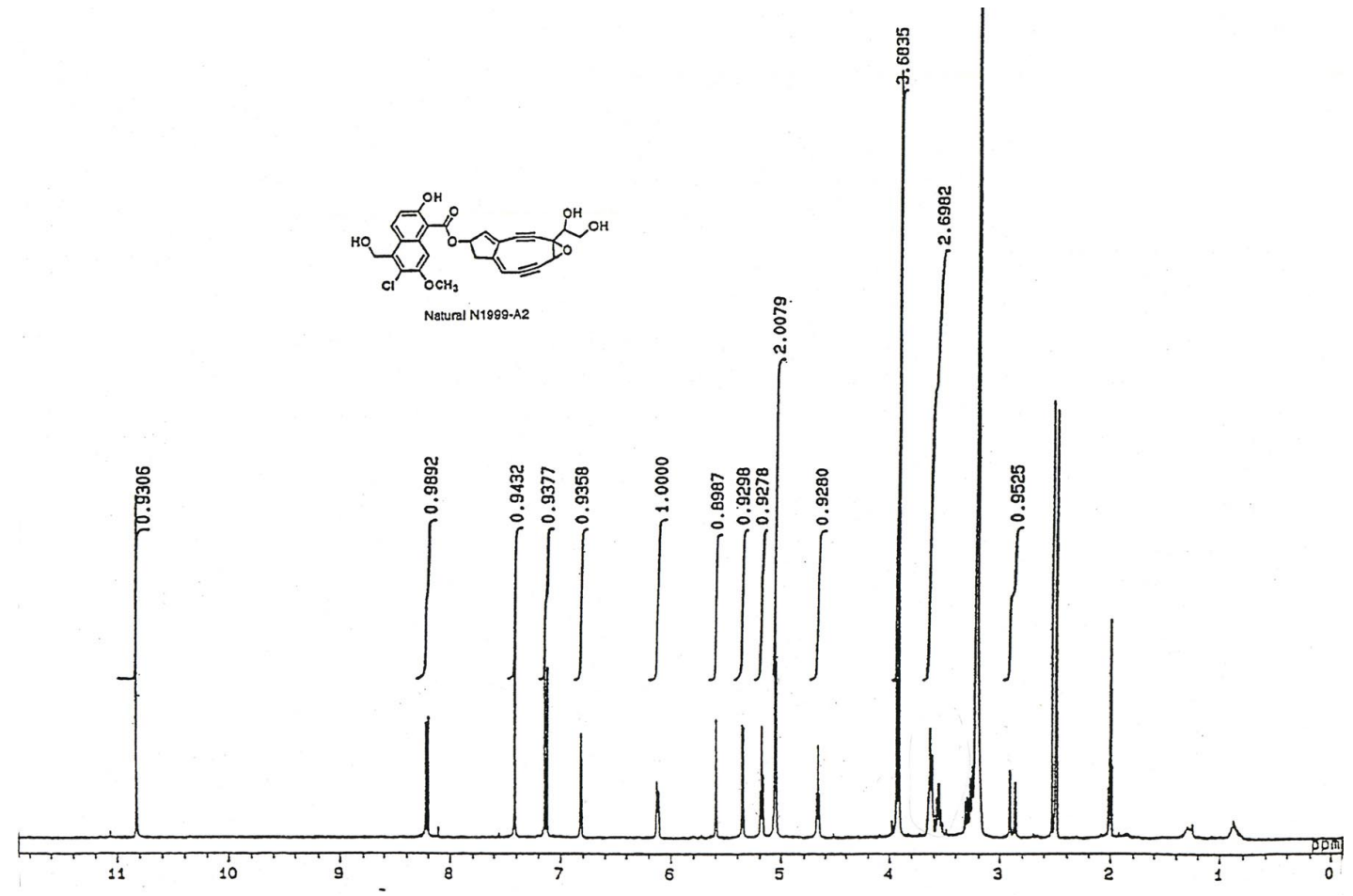

The ultraviolet spectrum below (right) was reproduced from Supporting Information, The First Total Synthesis of N1999A2: Absolute Stereochemistry and Stereochemical Implications into DNA Cleavage, J. Am. Chem. Soc. 2001, 123, 11294.
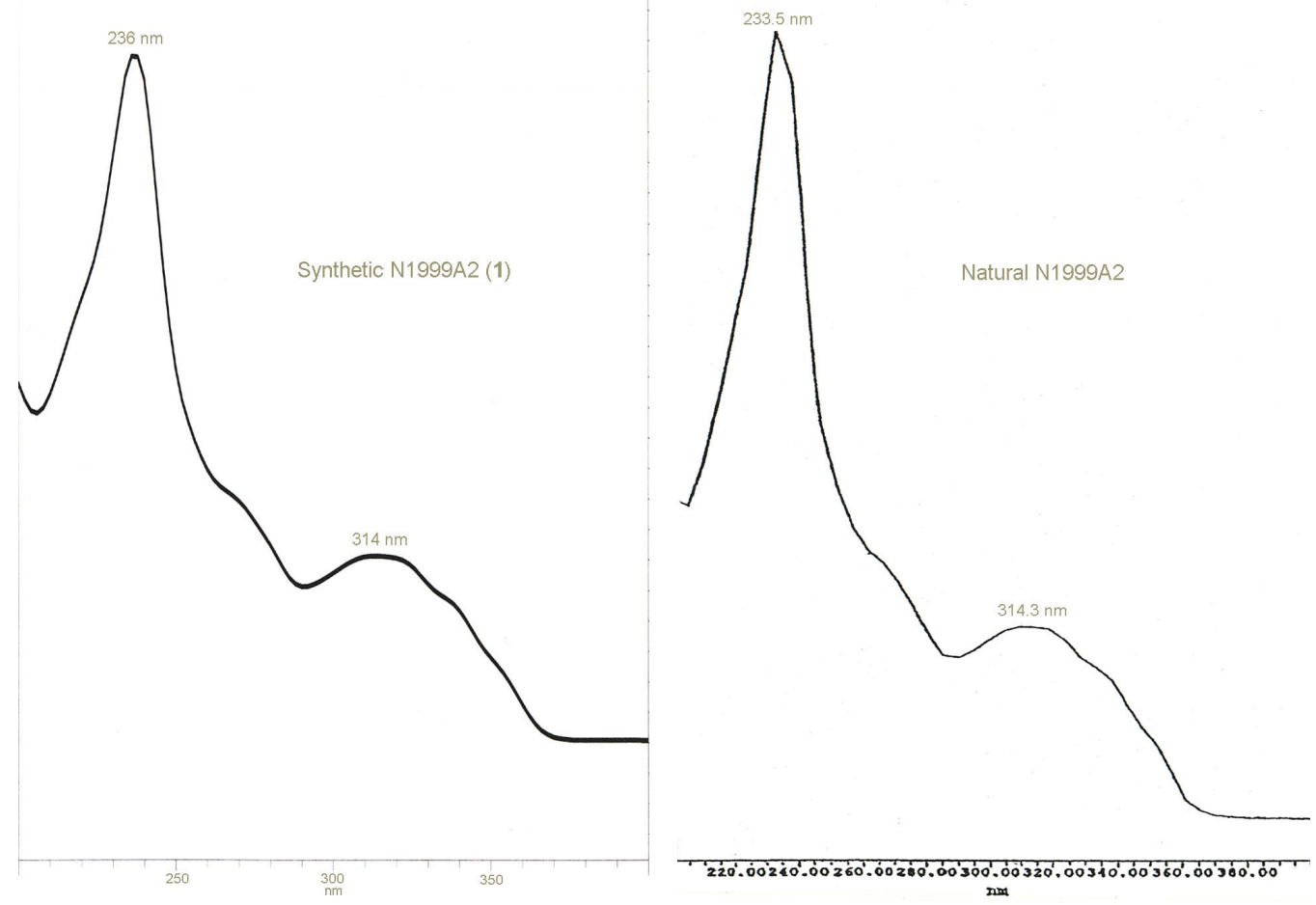
UV spectra of synthetic N1999A2 (1, left) and natural N1999A2 (right). 